Library, E.01 Al ni. b Jg.

dec $231970 \quad$ NBS TECHNICAL NOTE $\mathbf{5 4 5}$

\title{
Microchemical \\ Analysis Section:
}

Summary of Activities

July 1969 to June 1970

U.S. RTMENT OF MMERCE National Bureau of tandards 



\title{
UNITED STATES DEPARTMENT OF COMMERCE
}

Maurice H. Stans, Secretary

NATIONAL BUREAU OF STANDARDS - Lewis M. Branscomb, Director

\section{NBS TECHNICAL NOTE 545 ISSUED DECEMBER 1970}

Nat. Bur. Stand. (U.S.), Tech。Nate 545, 126 pages (Dec。1970) CODEN: NBTNA

\section{Microchemical Analysis Section: Summary of Activities, July 1969 to June 1970}

\author{
John K. Taylor, Editor \\ Microchemical Analysis Section \\ Analytical Chemistry Division \\ Institute for Materials Research \\ National Bureau of Standards \\ Washington, D.C. 20234
}

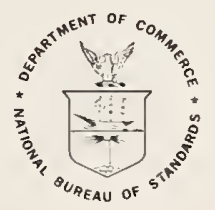

NBS Technical Notes are designed to supplement the Bureau's regular publications program. They provide a means for making available scientific data that are of transient or limited interest. Technical Notes may be listed or reierred to in the open literature.

For sale by the Superintendent of Documents, U.S. Government Printing Office, Washington, D.C., 20402. (Order by SD Catalog No. C 13.46:545). Price $\$ 1.25$. 

The Analytical Chemistry Division was established as a separate division at the National Bureau of Standards on September 1, 1963, and became part of the Institute for Materials Research in the February 1, 1964, reorganization. It consists at present of nine sections and about 100 technical personnel encompassing some 60 different analytical competences from activation analysis and atomic absorption to vacuum fusion and $\mathrm{x}$-ray spectroscopy. These competences, and in turn the sections which they comprise, are charged with research at the forefront of analysis as well as awareness of the practical sample, be it standard reference material or service analysis. In addition it is their responsibility to inform others of their efforts.

Formal publication in scientific periodicals is a highly important output of our laboratories. In addition, however, it has been our experience that informal, annual summaries of progress describing efforts of the past year can be very valuable in disseminating information about our programs. A word is perhaps in order about the philosophy of these yearly progress reports. In any research program a large amount of information is obtained and techniques developed which never find their way into the literature. This includes the "negative results" which are so disappointing and unspectacular but which can often save others considerable work. Of importance also are the numerous small items which are often explored in a few days and which are not important enough to warrant publication--yet can be of great interest and use to specialists in a given area. Finally there are the experimental techniques and procedures, the designs and modifications of equipment, etc。, which often require months to perfect and yet all too often must be covered in only a line or two of a journal article. 
Thus our progress reports endeavor to present this information which we have struggled to obtain and which we feel might be of some help to others. Certain areas which it appears will not be treated fully in regular publications are considered in some detail here. Other results which are being written up for publication in the journal literature are covered in a much more abbreviated form.

At the National Bureau of Standards publications such as these fit logically into the category of a Technical Note. In 1970 we plan to issue these summaries for all of our sections. The following is the sixth annual report on progress of the Microchemical Analysis Section.

W. Wayne Meinke, Chief Analytical Chemistry Division 


\section{PREFACE}

This report summarizes the current program and recent activities of the Microchemical Analysis Section of the Analytical Chemistry Division. This Section has the primary mission to contribute to the science and technology of the analysis of small samples and its program includes both fundamental studies to improve the precision, accuracy and sensitivity of analytical techniques, and developmental research to provide new and improved methods applicable to wide areas of materials characterization. As a closely related activity, the Section provides analytical measurements for various research programs of the Bureau and develops and characterizes Standard Reference Materials. Research projects are active in the following competence areas: gas analysis; polarography; microscopy; and conventional microchemical analysis.

A major effort of the Section during the year has been concerned with the analysis of standard reference materials. No effort has been spared to characterize these materials to a high degree of reliability. The use of such materials not only establishes benchmarks for industrial control but also provides a sound basis for inter-laboratory comparisons and for the intercomparison of measurement techniques and methodology. Such materials are regularly used in this laboratory to verify the accuracy of analytical procedures. They are referred to by an identification such as SRM 160b, for example. A catalog containing full descriptions of these materials may be obtained from the Office of Standard Reference Materials, National Bureau of Standards, Washington, D. C. 20234.

A number of analytical methods developed or modified for the analysis of standard reference materials as well as materials submitted by several NBS research projects are described briefly. Space limitations have dictated only a 
brief description of methods in most cases. It is hoped that such descriptions will be understandable to an experienced analyst. However, specific details of all work reported here will be made available to anyone interested in such matters. Some of the work discussed is a continuation of activities described in previous reports. Copies of the latter are available upon request in most cases.

In order to describe procedures, it has been necessary occasionally to identify commercial materials and equipment in this report. In no case does such identification imply recommendation or endorsement by the National Bureau of Standards, nor does it imply that the material or equipment is necessarily the best availalbe for the purpose.

John K. Taylor, Chief

Microchemical Analysis Section 
1. GAS ANALYSIS . . . . . . . . . . . . . . 1

A. Introduction ................. 1

B. Standard Reference Materials . . . . . . 2

1. Carbon Monoxide in Air. . . . . . . . 3

a. Method of Analysis . . . . . . . . 3

b. Method Evaluation . . . . . . . . 7

c. Method Modifications . . . . . . . 8

2. Sulfur Dioxide Permeation Tubes. . . . . 9

a. Calibration . . . . . . . . . 10

b. Results ............ . 18

c. Storage and Use . . . . . . . 22

d. Conclusions. • . . . . . . • . 29

3. Organic Materials. . . . . . . . . 30

C. High-Purity Gases............. . 31

D. Atmospheric Oxygen Determination . . . . . 36

E. Research Support Services. . : . . . . . . 36

1. Products from Polymer Degradation Studies. 37

2. Helium-3 Analysis. . . . . . . . . 38

3. Polywater............ . . 39

2. POLAROGRAPHIC ANALYSIS . . . . . . . . 41

A. Introduction ............. . 4 41

B. New Facilities ............ . 4 41

C. Major Constituent Analysis........ . 44

1. Copper and Zinc in Metal Foil. . . . . . 44

2. Copper and Nickel in Foils...... . 44

3. Antimony in Lead-base Bearing Metal. . . 45

4. Cadmium in Cadmium Cyclohexanebutyrate

(SRM 1053a)........... 46

D. Minor Constituent Analysis: : : : : : : 46

E. Small Sample Analysis. . . . . . . . . 48

F. Trace Analysis ............. . 49

l. Reagent Analysis . . . . . . . . . 49

a. Perchloric Acid. . . . . . . . . 49

b. Nitric Acid and Hydrochloric Acid. • . 5l

c. Ammonium Hydroxide . . . . . . . . 52

2. Environmental Analysis . . . . . . . 53

a. Laboratory Air . . . . . . . . 53

b. Industrial Air . . . . . . . . 55

3. Lead in Fish Protein Concentrate. . . . 56

4. Copper and Zinc in Blood Plasma Processing Intermediates. . . . . . . . . 56

5. Lead, Bismuth and Tellurium in Special Steels . . . . . . . . . . 57

6. Titanium in special Steels and Ductile Irons. . . . . . . . . . . 59 
7. Nickel, Iron, and Titanium in Glass. . . 61

3. MICROSCOPIC AND CLASSICAL MICROCHEMICAL ANALYSIS. 70

A. Introduction . . . . . . . . . . . 70

B. Microchemical Standards . . . . . . . 70

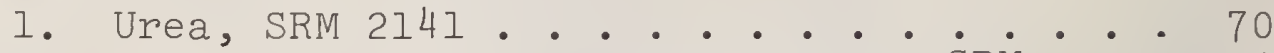

2. Intercomparison of Microchemical SRMS. . 73

3. Orchard Leaves, SRM 1571 . . . . . . 74

C. Ultramicrochemical. Standards . . . . . . . 77

D. Research Material Analysis . . . . . . . 78

1. Copper-Nickel and Copper-Palladium Alloys. 78

2. Tungsten-Rhenium Alloys. . . . . . . 79

3. Thermochemical Reaction Evaluation... . 80

4. Fluorinated Copolymer Analysis . . . . 81

4. Air pollutant anAlYSis . . . . . . . . . . 82

A. Nature of the Analytical Problem . . . . . . 82

l. Interpretation . . . . . . . . . 83

2. Sampling . . . . . . . . . . . 85

3. Physical Analysis. . . . . . . . . . 86

4. Metrology. . . . . . . . . . . 86

5. Chemical Analysis. . . . . . . . 86

B. State of the Art of Chemical Analysis. . . . 88

C. State of the Art of Standard Reference

Materials ............... 89

D. Analytical Methods for Specific Poliutants: 91 1. Gaseous Pollutants........ . 9 94

a. Sulfur Dioxide . . . . . . . . . 94

b. Hydrogen Sulfide . . . . . . . . 95

c. Nitrogen Oxides . . . . . . . . . 95

d. Ozone and Oxidants . . . . . . . 96

e. Volatile Fluorides and Hydrogen

Fluoride • . • • • • • • . • . 97

f. Carbon Monoxide .. . . . . . . 98

g. Hydrocarbons . . . . . . . . . . 98

h. Other Pollutants . . . . . . . . 99

i. Other Techniques . . . . . . . . 99

2. Particulate Pollutants . . . . . . 100

a. Dustfall and Sootfall ...... 100

b. Air-borne Particulates . . . . . 101

3. Analysis of Single Particles...... 104

E. Summary. . . . . . . . .... 105

5. PERSONNEL AND ACTIVITIES . . . . . . . . 107

A. Personnel Listing. . . . . . . . . . . 107

B. Publications... . . . . . . . . . 107 
C. Talks................. 107

D. Committee Activities. . . . . . . . . 109

E. Award . . . . . . . . . . . . . . 110

F. Standard Reference Materials Analyses . . . 110

6. REFRRENCES ..................... 112

\section{LIST OF FIGURES}

FIGURE NO.

$\underline{P A G E}$

1. Double focusing mass spectrometer. . . . . 2

2. Schematic diagram of apparatus for determination of carbon monoxide . . . . . 4

3. Apparatus for determination of carbon monoxide

4. Chamber for maintaining permeation tubes at constant temperature . . . . . . . . . Il

5. Gravimetric calibration of permeation tubes. 12

6. Predicted effect of moisture on $\mathrm{SO}_{2}$ permeation rate. . . . . . . . . . . 15

7. Predicted decrease of vapor pressure of $\mathrm{SO}_{2} \cdot 16$

8. Change in weight of permeation tube after low-temperature storage. . . . . . . 25

9. Correlation of permeation rate with tube sequence . . . . . . . . . . . .

10. Apparatus for absorption of major constituent in a gas mixture. . . . . . 32

11. Device for concentrating impurities in carbon dioxide......... . . 34

12. Device for minimizing contamination when sampling from gas cylinder . . . . . . .

13. Three-unit clean air module . . . . . . 42

14. Single-unit clean air module. . . . . . 43

15. The analytical system for environmental measurements 
1. Reliability of gravimetric calibration . . 20

2. Typical calibration uncertainties. . . . . 21

3. Storage behavior . . . . . . . . . 22

4. Recovery of rate after removal from low temperature storage. . . . . . . . . . 23

5. Temperature cycling behavior . . . . . 26

6. Short term temperature effects...... . 28

7. Atmospheric oxygen determination . . . . 37

8. Gaseous products from thermally degraded $\mathrm{H}-\mathrm{film}$. . . . . . . . . . . . 38

9. Gas analytical services........ . 40

10. Analysis of $\mathrm{Cu}-\mathrm{Ni}$ foils... . . . . . 45

11. Cadmium in SRM 1053a. . . . . . . . 47

12. Aluminum in ferrosilicon, SRM 59a . . . . 48

13. $\mathrm{Fe}, \mathrm{Cu}, \mathrm{Pb}$, and $\mathrm{Cd}$ in perchloric acid. . . 50

14. Total EDTA-complexed metals in perchloric acid............ . . 51

15. Analysis of ammonium hydroxide . • . . . 52

16. Particulates in laboratory air. . . . . . 53

17. Particulates in clean room air. . . . . . 54

18. Fallout in laboratory and clean room air. . 54

19. Copper and zinc in sodium lactate and sodium chloride solutions. . . . . . . . 57

20. Lead and bismuth in special steels... . . 58

21. Tellurium in special steels. . . . . . . 59

22. Titanium in ductile iron SRM 1140... . . 60

23. Titanium in SRM 365 and SRM 1265..... . 60

24. Nickel in 500-ppm glass......... 62

25. Nickel in l-ppm glass... . . . . . . 63

26. Iron and titanium in base glass. . . . . 64

27. Iron and titanium in 0.02-ppm glass. . . . 65

28. Iron and titanium in 50-ppm glass..... 66

29. Iron in 500-ppm glass......... 68 
TABLE NO.

$\underline{\text { PAGE }}$

30. Titanium in 500-ppm glass. . . . . . . 69

31. Determination of nitrogen in urea SRM 2141. 71

32. Homogeneity studies of urea SRM 2141 • • • 72

33. Purity of microchemical SRMs . . . . . . 73

34. Nitrogen in orchard leaves, SRM 1571.. • . 75

35. Phosphorus in orchard leaves, SRM 1571 . . 76

36. Microscopic Nessler spot test. . . . . . 78

37. Standard reference materials related to environmental analysis. . . . . . . . . 91

38. Analysis of gaseous pollutants . . •. . . 93

39. Particulate characterization.. . . . . . 93

40. Expected schedule of appearance of NAPCA

air quality criteria documents . . . . . 94 

MICROCHEMICAL ANALYSIS SECTION: SUMMARY OF ACTIVITIES

JULY 1969 to JUNE 1970

Edited by John K. Taylor

This report describes the research activities and scientific programs of the Microchemical Analysis Section of the Analytical Chemistry Division of the National Bureau of Standards, Institute for Materials Research during the period July 1969 to June 1970. General Activities are reviewed in areas of gas analysis polarography, chemical microscopy, and classical microchemical analysis. Research activities described in some detail include: preparation and analysis of carbon monoxide mixture for standard reference materials; evaluation of sulfur dioxide permeation tubes as analytical standards; polarographic methods for determination of trace elements in a number of materials; certification of urea as a microchemical standard. A chapter reviewing the state-of-the art of analysis for air pollutants is also included.

Key words: Air pollutant analysis; chemical analysis; gas analysis; microchemical analysis; microscopic analysis; polarographic analysis.

\section{GAS ANALYSIS}

A. Introduction

The Gas Analysis Laboratory is concerned with the development of new or improved methods of gas analysis, mainly by mass spectrometry. The development of standard reference materials and their certification and the analysis of a wide variety of research materials are important functions. Emphasis on both methodology and SRMs for air pollution analyses has been an increasing activity over the past several years and especially so during the past year. A program to develop new concepts for SRMs for air pollutant analysis and for the evaluation of analytical methodology has been initiated in cooperation with the National Air Pollution Control Administration. 
The laboratory acquired a new double focusing mass spectrometer during the year. The extended mass range (12$3000 \mathrm{amu}$ ) and heated probe (up to $400^{\circ} \mathrm{C}$ ) have greatly facilitated the rapid analysis of solid and liquid organic materials of relatively low vapor pressure. In addition, the moderately high resolution ( 1 part in $3000 \mathrm{amu}$ ) has been of value in the examination of gases containing molecules producing ions of the same nominal masses, such as carbon dioxide and nitrogen. The apparatus is shown in Figure 1.

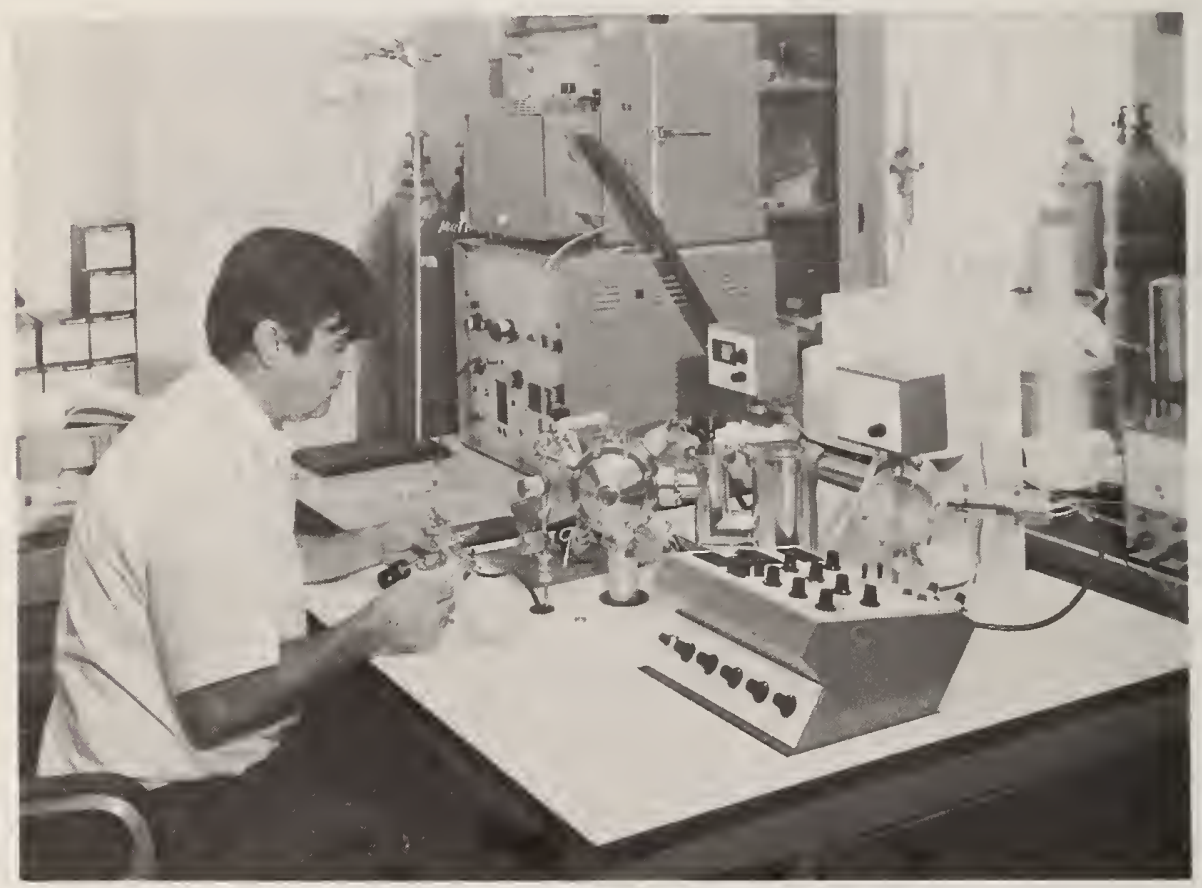

Figure 1. Double focusing mass spectrometer

B. Standard Reference Materials

A program to develop standard reference materials for gas analysis was initiated in 1966. The first of these, issued in 1967, consisted of mixtures of carbon dioxide in nitrogen, (SRM 1601, SRM 1602, SRM 1603), at $\mathrm{CO}_{2}$ levels close to concentrations of the normal atmosphere. In the following years, a series of standards was prepared containing oxygen 
at trace-to-atmospheric concentrations (SRM 1604, SRM 1605, SRM 1606, SRM 1607, SRM 1608, SRM 1609).

The first gaseous SRMs specifically prepared for air pollution analysis were issued in 1968. These consisted of a series of methane-in-air standards, (SRM 1610, SRM 1611, SRM 1612, and SRM 1613), containing approximately 1000, 100 , 10 and 1 ppm methane, respectively. During the past year, a series of standards containing carbon monoxide in air has been under development and is nearing completion. In addition, a program for the development of SRMs for pollution studies, based on new concepts, has been initiated in cooperation with the National Air Pollution Control Administration. These activities are described in the following sections.

1. Carbon Monoxide in Air

The importance of carbon monoxide as an atmospheric pollutant has prompted a program to produce a series of standard reference materials containing known amounts of this gas. Such SRMs should be useful to calibrate measurement instruments and procedures utilizing non dispersive infrared measurements and gas chromatography. The problem of certifying these materials is twofold - - the accurate measurement of the co content, and the question of stability of composition during extended periods of storage. Some results of the investigation of both aspects of the problem are described here.

a. Method of Analysis. Past attempts in this laboratory to determine $\mathrm{CO}$ in air by oxidizing the $\mathrm{co}$ to $\mathrm{CO}_{2}$, which is then determined mass spectrometrically have not been entirely successful. Specifically, this method fails at concentrations of CO similar to those expected in the atmosphere. Stevens [1] described a method whereby the co, separated from oxygen, nitrogen, and hydrocarbons by a chromatographic technique was reduced with hydrogen on a nickel catalyst to form methane which was subsequently determined by a flame ionization technique. It was thought that the above method, 
or a variation thereof, would be suitable for determining co with sufficient accuracy for certification of standard reference gases whose $\mathrm{CO}$ contents were to range from 1000 to $10 \mathrm{ppm}$.

The nickel catalyst was prepared by evaporation to dryness of a saturated solution of nickelous nitrate and $30 / 40$ mesh fire brick. A six inch column of this material was packed in $3 / 8$ inch stainless steel tubing and was heated to $500{ }^{\circ} \mathrm{C}$ using a tube furnace. The catalyst was activated prior to use and, periodically during use, by oxidation with oxygen, followed by reduction with hydrogen. The catalyst tube was incorporated in the system as shown in Figure 2. The gas mixing system upstream of the catalyst tube facilitated the blending of the hydrogen-helium carrier gas in proportions

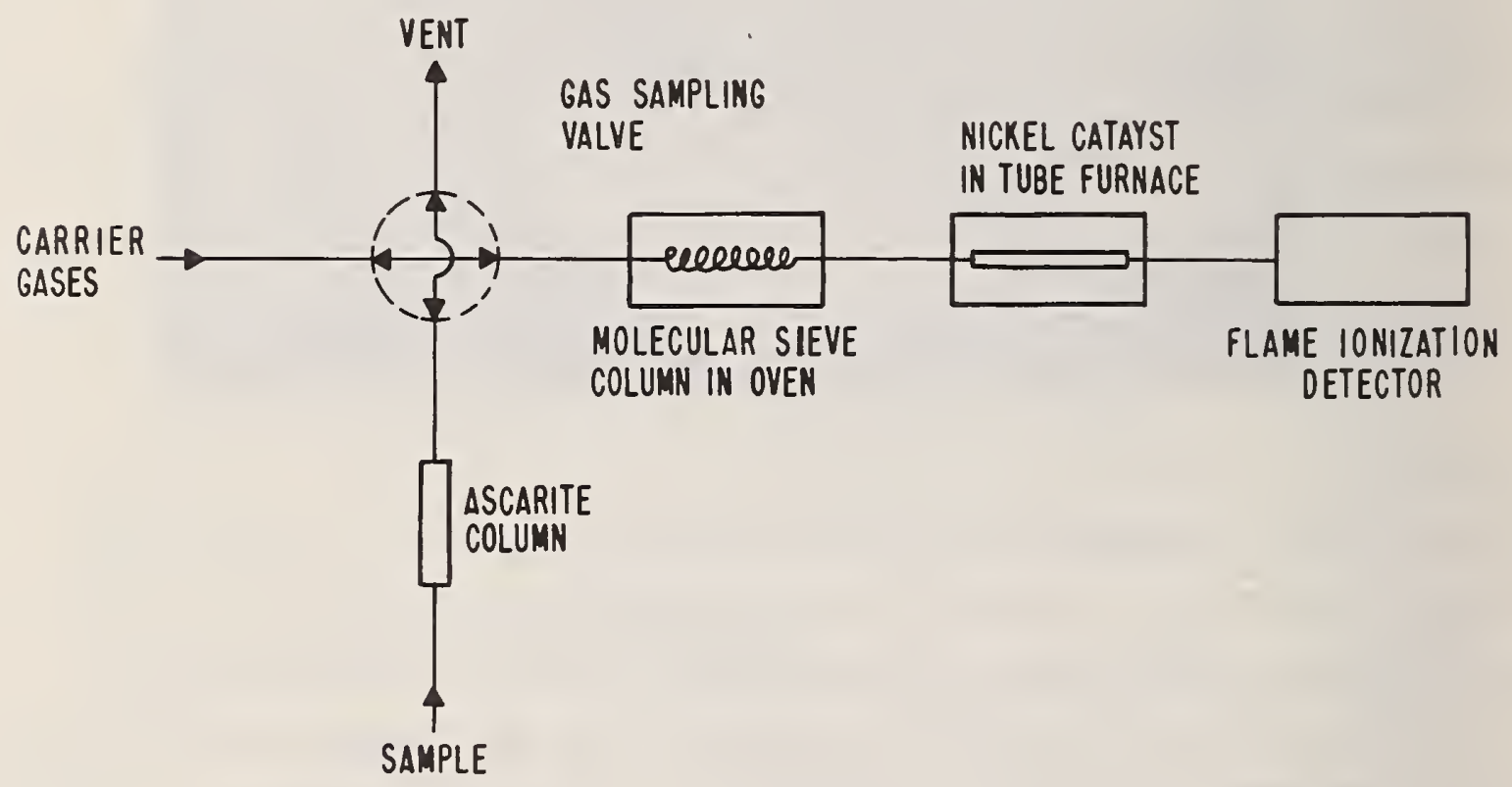

Figure 2. Schematic diagram of apparatus for determination of carbon monoxide. 
to give maximum sensitivity. The gas sampling valve admitted a sample of reproducible volume to the system. A flame ionization hydrocarbon detector was connected to the outlet end of the catalyst tube. The apparatus is shown in Figure 3.

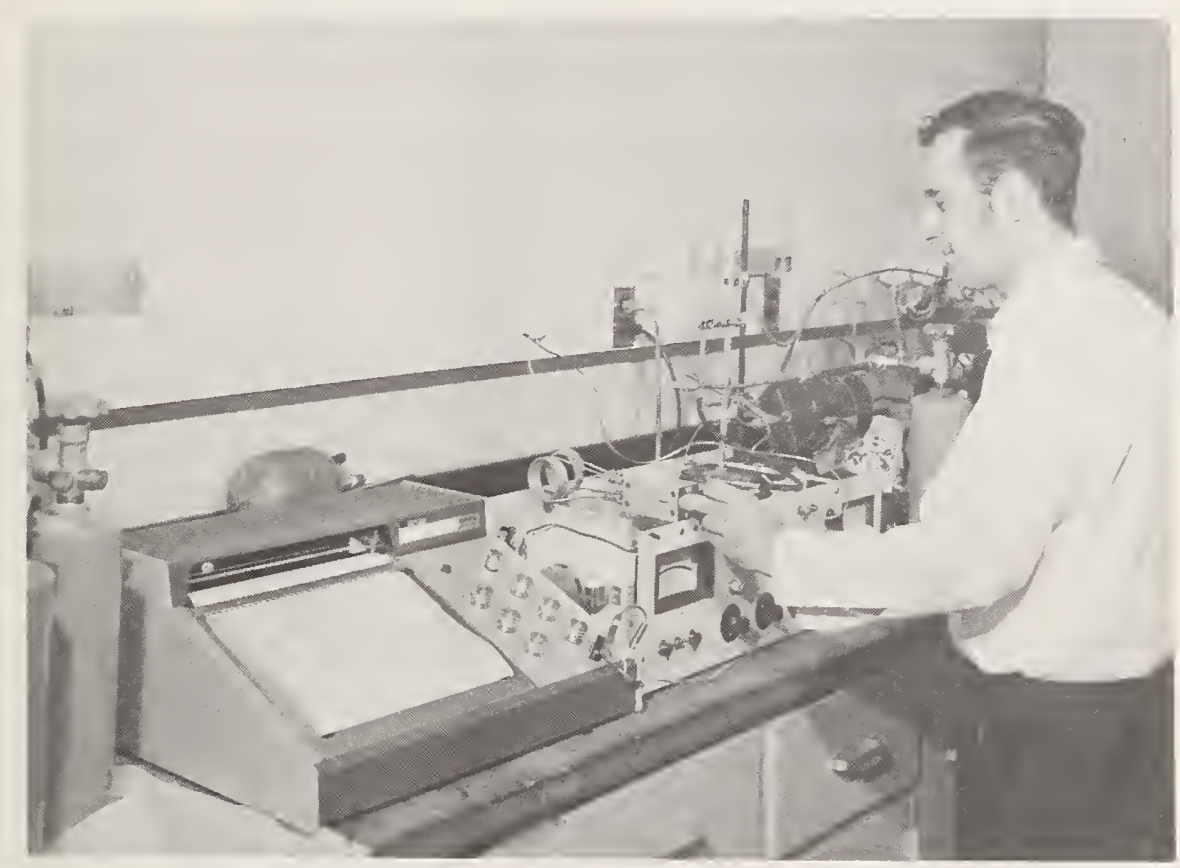

Figure 3. Apparatus for determination of carbon monoxide.

Several mixtures of $\mathrm{CO}$ in air and $\mathrm{CO}$ in nitrogen ranging from 13 to $1300 \mathrm{ppm}$ CO were available for testing the method. Briefly, it was found that a helium flow rate of about $7 \mathrm{~cm}^{3}$ / minute, a hydrogen flow rate of about $25 \mathrm{~cm}^{3} /$ minute, and a catalyst temperature of about $500^{\circ} \mathrm{C}$ gave maximum response from $1 \mathrm{~cm}^{3}$ of a $\mathrm{CO}$ mixture injected into the gas stream by the gas sampling valve. Any $\mathrm{CO}_{2}$ in the mixture which would be reduced on the catalyst to $\mathrm{CH}_{4}$, was removed by placing a 6-in column of Ascarite in the line between the sample gas cylinder and the gas sampling valve. 
Comparison of $\mathrm{CO}$ in nitrogen mixtures with $\mathrm{CO}_{2}$ or $\mathrm{CH}_{4}$ in nitrogen mixtures of approximately the same concentration gave results which were reproducible but which were 4-5 percent low. Results with mixtures of $\mathrm{CO}$ in air were erratic due to effects of oxygen upon the catalyst.

Since analyses of $\mathrm{CO}$ in air were required, the oxygen had to be separated from the $\mathrm{CO}$ before it passed over the catalyst. Accordingly, a 6 foot chromatographic column of $1 / 4$ in copper tubing containing 60/80 mesh $5 X$ molecular sieve was placed in a thermostatically controlled oven in the system between the catalyst and the gas sampling valve. This provided adequate separation of both nitrogen and oxygen from CO, however, methane was not separated. Accordingly, methane had to be determined in all co mixtures which might be analyzed and its response subtracted in order to obtain the actual co response.

Variation of helium and hydrogen flow rates and column and catalyst temperatures established values of these parameters which gave optimum peak height and separation of the co peak. The hydrogen and helium flow rates were 10 and $8 \mathrm{~cm}^{3} / \mathrm{min}$, respectively. The chromatographic column was operated at $85^{\circ} \mathrm{C}$ and the catalyst was maintained at $450^{\circ} \mathrm{C}$. A series of six mixtures of $C O$ in air was compounded in $1.5 \mathrm{ft}^{3}$ cylinders using $C O$ which had been analyzed for impurities and diluent air which was analyzed specifically for $\mathrm{CO}$ and methane. (These mixtures were to be transferred to smaller cylinders and used as standard reference materials after analysis.) The concentrations of $\mathrm{CO}$ in the mixtures calculated from the pressures of the component gases were 1017, 534, 304, 102, 45.1, and 10.1 ppm CO, respectively. The response, in terms of signal versus concentration, was reproducible at any concentration but was not linear over the observed range. A co detecting tube inserted in the exhaust line from the flame ionization detector showed no evidence of $\mathrm{CO}$, therefore complete con- 
version was assumed. Laboratory standards of $\mathrm{CO}$ in air were prepared in small cylinders by gravimetry to which comparisons could be made as the final step in the analysis.

\section{b. Method Evaluation. Initially, three}

laboratory standards were compounded gravimetrically with co concentrations of 1002, 101.7, and 10.25 ppm CO; respectively. The use of these laboratory standards whose concentrations were within about 1 percent of those of the corresponding standard reference material gases, should have yielded valid calibrations for the SRMs. Comparisons made between three SRMs and the corresponding laboratory standard gases gave the results below.

$\begin{array}{cc}\text { Concentration (measured) } & \text { Concentration (mixing data) } \\ (\mathrm{ppm} \mathrm{CO}) & (\mathrm{ppm} \mathrm{CO}) \\ 1021 & 1017 \\ 102.5 & 102 \\ 9.45 & 10.1\end{array}$

Responses from the other three standard reference gases fell at approximately the proper position on a response vs. concentration plot. The discrepancy between analytical and mixing data at the $10 \mathrm{ppm}$ level was at first thought to be due to an error in the mixing data for the standard reference gas. Accordingly, another mixture of $\mathrm{CO}$ in air was prepared with a calculated $\mathrm{CO}$ content of $10.26 \mathrm{ppm}$. Comparison of this with the corresponding laboratory standard indicated a CO concentration of $10.39 \mathrm{ppm}$.

In order to confirm the $\mathrm{CO}$ concentrations of the other standard reference gases, laboratory standards containing 533.9 and $44.7 \mathrm{ppm} \mathrm{CO}$, respectively, were prepared gravimetrically. Comparison of these with the standard reference gases of similar concentration gave discrepant results which were about 2 percent low at the $500 \mathrm{ppm}$ level and about 5 percent low at the $50 \mathrm{ppm}$ level. The standard reference gases had been prepared about four months before comparison to the freshly prepared laboratory 
standards. The discrepancies observed suggested an instability of co-air mixtures stored in mild steel cylinders. The deviation between concentrations from analytical and mixing data at the $10 \mathrm{ppm}$ co level could also be explained as due to loss of co rather than an error in mixing data.

There is other evidence that $\mathrm{CO}$ is not stable when stored in mild steel cylinders. Some of the standard reference gas at the $100 \mathrm{ppm}$ co level was transferred from its original large cylinder to small mild steel cylinders. Measurements made two months after the transfer showed the co content of the gas in the small cylinders had decreased by 2 to 3 percent. It was found that heating small cylinders containing $\mathrm{CO}$ in air at the 300 and $100 \mathrm{ppm}$ levels at $60{ }^{\circ} \mathrm{C}$ for 6 days reduced the $\mathrm{CO}$ content by about 3 and 7 percent, respectively. Mixtures of about $50 \mathrm{ppm} C O$ in nitrogen also showed deterioration when heated in mild steel cylinders at $60{ }^{\circ} \mathrm{C}$ for several days.

The apparent loss of $\mathrm{CO}$ in mild steel containers is probably the result of oxidation of $\mathrm{CO}$ to $\mathrm{CO}_{2}$. Whether this results from direct oxidation by gaseous oxygen or whether it occurs indirectly in the presence of iron oxides is not known. It suggests, however, that a possible solution to the problem of instability may lie in the use of a less reactive container such as one constructed of stainless steel. Accordingly, CO in air mixtures at the 10 and $1000 \mathrm{ppm}$ level were stored in stainless steel containers at $60{ }^{\circ} \mathrm{C}$ for two weeks without detectable losses of CO. Aging tests of longer duration are now in progress.

c. Method Modifications. During the course of the investigation described above, several modifications of the apparatus were made. A valve with $5 \mathrm{ml}$ sampling loops was installed which was also able to withstand higher carrier gas pressure than the original valve. The 6-foot copper chromatographic column was replaced by a 12-foot, 1/4 in 
stainless steel column, to better separate the CO and methane peaks. The optimum flow rates for hydrogen and helium with this column were 12 and $8 \mathrm{~cm}^{3} / \mathrm{min}$, respectively, at a column temperature of $85^{\circ} \mathrm{C}$.

A series of mixtures of $\mathrm{CO}$ in air, ranging in concentration from 10 to $1000 \mathrm{ppm}$, was prepared in stainless steel cylinders. Not only do these mixtures appear to be stable but a linear response was obtained when they were measured with the modified apparatus. The non-linearity previously observed with the copper column could have resulted from oxidation of small amounts of $\mathrm{CO}$ to $\mathrm{CO}_{2}$ by traces of copper oxide present on the walls, at the $85^{\circ} \mathrm{C}$ column operation temperature.

The linear response with the improved apparatus demonstrated that the efficiency of reduction of carbon monoxide to methane is independent of concentration of the former. Therefore, it is possible to verify the efficiency of the reduction reaction by the use of NBS Standard Reference Materials, Methane-in-Air, SRM 1610: SRM 1611, SRM 1612, SRM 1613. These measurements are now in progress and indicate that the efficiency of the catalytic reduction of $\mathrm{CO}$ to methane, under the conditions described above which are essentially those recommended by Stevens [1], is essentially 100 percent on the entire concentration range. The two series of SRMs, CO-in-Air and Methanein-Air, will be certified as to their inter-comparability so that they may be used to verify analytical procedures, such as the Stevens method [1].

2. Sulfur Dioxide Permeation Tubes

The use of plastic tubes containing liquid sulfur dioxide, from which the sulfur dioxide effuses at a rate dependent only on temperature, has been proposed as a method for producing known concentration of sulfur dioxide in controlled atmospheres [2]. Sulfur dioxide is too reactive to allow preparation and storage of low concentration mixtures in metallic cylinders without the danger of subsequent changes in the concentration. 
Consequently, these devices known as effusion or permeation tubes offer the most promising method for the production of known concentrations in the part per billion to part per million range.

The usefulness of these devices has been established [3] but their behavior over long periods of time and under conditions similar to those encountered in normal use has not been fully studied. A study was made, therefore, to follow a group of "representative" tubes throughout typical circumstances which might be encountered during calibration and use.

a. Calibration. Initially, lots of tubes from two different producers were included in the major portion of the evaluation and later two different lots from the same suppliers were studied in regard to their calibration only.

The calibration was performed by periodically weighing the tubes, maintained at a constant temperature, after measured time intervals. The rate of permeation is simply the weight change during this time divided by the time. To obtain accurate rates, the temperature of the tubes was maintained constant to about $\pm 0.01{ }^{\circ} \mathrm{C}$ during storage between weighing. The tubes were stored in a chamber similar to that shown in Figure 4. Air, previously passed through a column of activated charcoal, enters the chamber at the bottom after passing through the heat exchanger tube. The whole vessel is immersed in a thermostatted bath to the level shown. Measurement of the temperature of the bath and of the interior of the chamber under actual conditions of storage indicated a difference of less than 0.005 degrees at the fastest flow rates employed. When large numbers of tubes were contained in a chamber, the flow rate was at least 2 liters per minute. With fewer tubes slower rates were employed. The time between weighings varied from about 20 hours to four or five days. The time was chosen such that the weight loss was more than two orders of magnitude greater than the estimated error involved in the weighing. Because of the large number of 


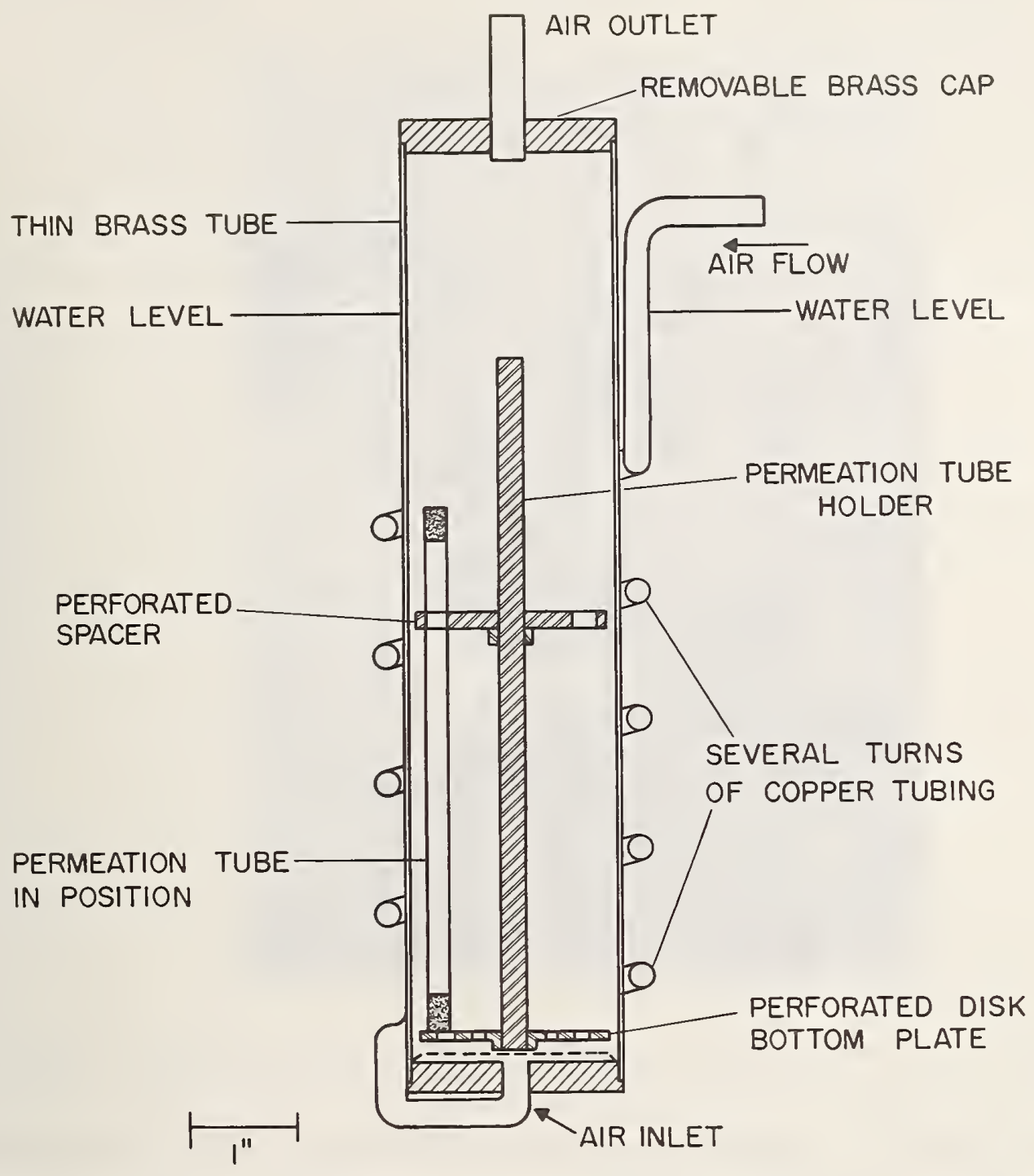

Figure 4. Chamber for maintaining permeation tubes at constant temperature.

tubes which were to be weighed, and because the temperature at the time of weighing often differed from the temperature of storage, the weighing procedure had to be simplified as much as possible. 
A single pan microbalance, readable to $1 \mu g$ was used for all weighings (Fig. 5). Losses in weight between weighings

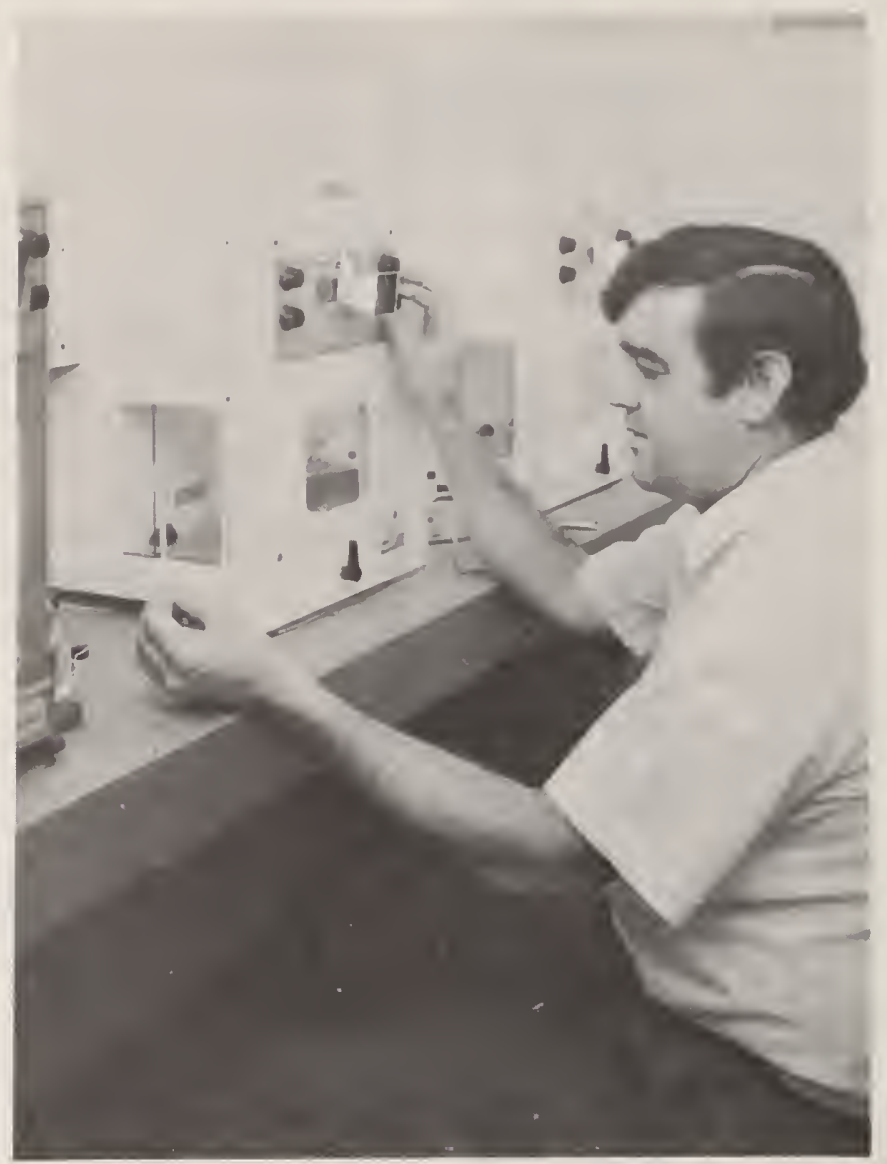

Figure 5. Gravimetric calibration of permeation tubes.

were always greater than $1 \mathrm{mg}$. The method of handling tubes did not produce an error in terms of accumulated weight of more than $10 \mu \mathrm{g}$ for 20 weighings. This quantity is quite small in terms of the total weight loss of about 30 to $70 \mathrm{mg}$ during this period. Thus, it was concluded that tubes could be weighed rapidly without special precautions, thereby reducing the amount of time that tubes were exposed to temperatures differing from that of the storage chambers.

While it could be shown that direct handling of the tubes introduced only a small error in the weighing, it was further necessary to determine whether the type of direct 
handling employed in weighing could affect the rate of los of sulfur dioxide in a manner that would not easily be detected during calibration. Such an effect could arise from an accumulation of grease or dust in the pores of the Teflon. Surface dirt would probably have little or no effect on the rate of passage of sulfur dioxide but any foreign matter which could effectively block the pore spaces in the Teflon could influence the effusion rate. In practice, if tubes are used with reasonable care to prevent contact with dirty surfaces, the only major source of contamination would be from the fingers of the person weighing or otherwise handling the tubes. An accumulation of a significant amount of oil or grease would require constant mishandling and carelessness on the part of the operator. Small amounts, however, might accumulate through normal handling.

A simple, but probably valid experiment was prepared to determine whether accumulation of grease could be a significant source of error. The concentration of sulfur dioxide produced by a single tube in an air stream at a fixed flow rate and temperature was determined using a flame photometric sulfur analyzer [4]. The tube was then wiped with a hydrocarbon grease over 25 percent of its surface area and the concentration of $\mathrm{SO}_{2}$ produced in the air stream was redetermined. Since no change was observed when 25 percent of the surface was treated, the entire surface was next treated similarly. The surface was rubbed vigorously with grease, then the excess was wiped off. Again, no change in the concentration was observed indicating that this type of contamination does not affect the rate of transport of sulfur dioxide through the Teflon. Another source of error, but one which is much less easily evaluated, is the effect of the passage of water from the atmosphere into the sulfur dioxide within the tube. To evaluate this, several tubes were immersed in distilled water for periods up to three days. The rate of effusion of 
sulfur dioxide did not appear to be any different after this treatment when compared instrumentally with the rate before. However, if the rate of transport through the Teflon for a particular substance is determined largely by the vapor pressure of the substance then long-term exposure to an atmosphere partially saturated with water vapor could have appreciably greater effect than short-term exposure to liquid water. This is confirmed to some extent by the observation that the rate of loss of sulfur dioxide from a tube is independent of the ratio of liquid to vapor provided that two phases do exist. The rate of loss of water from tubes fabricated from similar tubing, that is, tubing of equal porosity to sulfur dioxide, is about 2 percent of that for sulfur dioxide. Accordingly, we can make a rough estimate of the total effect to be expected on exposure of a tube to ambient atmospheres containing moisture for long periods of time.

Sulfur dioxide permeation tubes are not usually allowed to remain in the atmosphere for long periods of time. They are ordinarily stored in closed containers with a dessicant or refrigerated at low temperature. In either case, it is unlikely that they would be in contact with a high concentration of water vapor for any but a brief period of time during their useful life. However, tubes may be used for the production of $\mathrm{SO}_{2}$ concentrations using ambient air, although a pre-drying of the air is recommended. The following considerations have been made to estimate the consequences of such a practice.

Consider a tube of typical effusion rate and assume that throughout its useful life it is constantly exposed to an atmosphere with a relative humidity of 25 percent at $25^{\circ} \mathrm{C}$. Water vapor will continually pass into the tube countercurrent, so to speak, to the sulfur dioxide. This passage will continue until the effective water vapor pressure within the tube is about $6 \mathrm{~mm}$. The solubility of the water in sulfur 
dioxide is variously reported but it is probably about 2.5 weight percent water [5]. (This pressure would not be reached in the vapor phase until the sulfur dioxide was nearly saturated with water.) The reduction in vapor pressure, and presumably the permeation rate, at saturation and where water would no longer migrate would be about 5 percent of the rate if water were not present. Because of the difference in the rate of permeation of the two species it is of interest to predict at approximately what time in the life of a tube that this effect would seriously alter the effusion rate. Figure 6 shows the rate of weight loss in micrograms per day

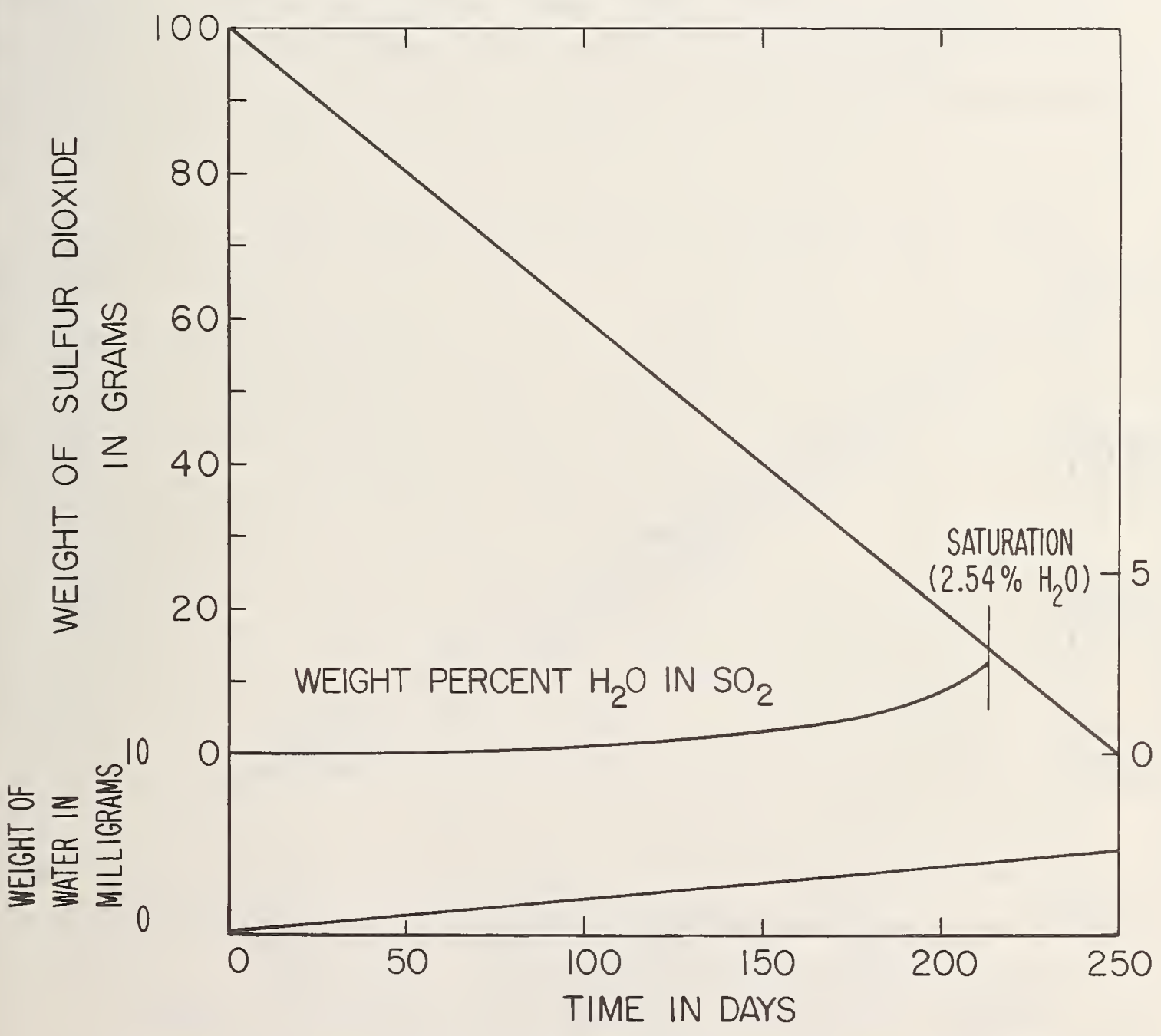

Figure 6. Predicted effect of moisture on $\mathrm{SO}_{2}$ permeation rate. 
of sulfur dioxide from a typical tube, the predicted weight of water gained by the tube during the same time and a superimposed curve of the weight percent of water in the liquid sulfur dioxide. Figure 7 is a representation of the decrease in vapor pressure in a system, sulfur dioxide-water, where the sulfur dioxide is less than saturated with water. This was derived from the vapor pressure data of Maass and Maass [6] and the solubility values of Daisaboro Murakumi and Nichiro Tokura [7]. The composition of the vapor as calculated from Raoult's law is uncertain because water is probably present in the sulfur dioxide as the hydrate of sulfurous acid, $\mathrm{H}_{2} \mathrm{SO}_{3} 6 \mathrm{H}_{2} \mathrm{O}$ [8]. At a weight concentration of water of 2.49 percent, the system is saturated and a second water-rich phase will separate: Up to this point,

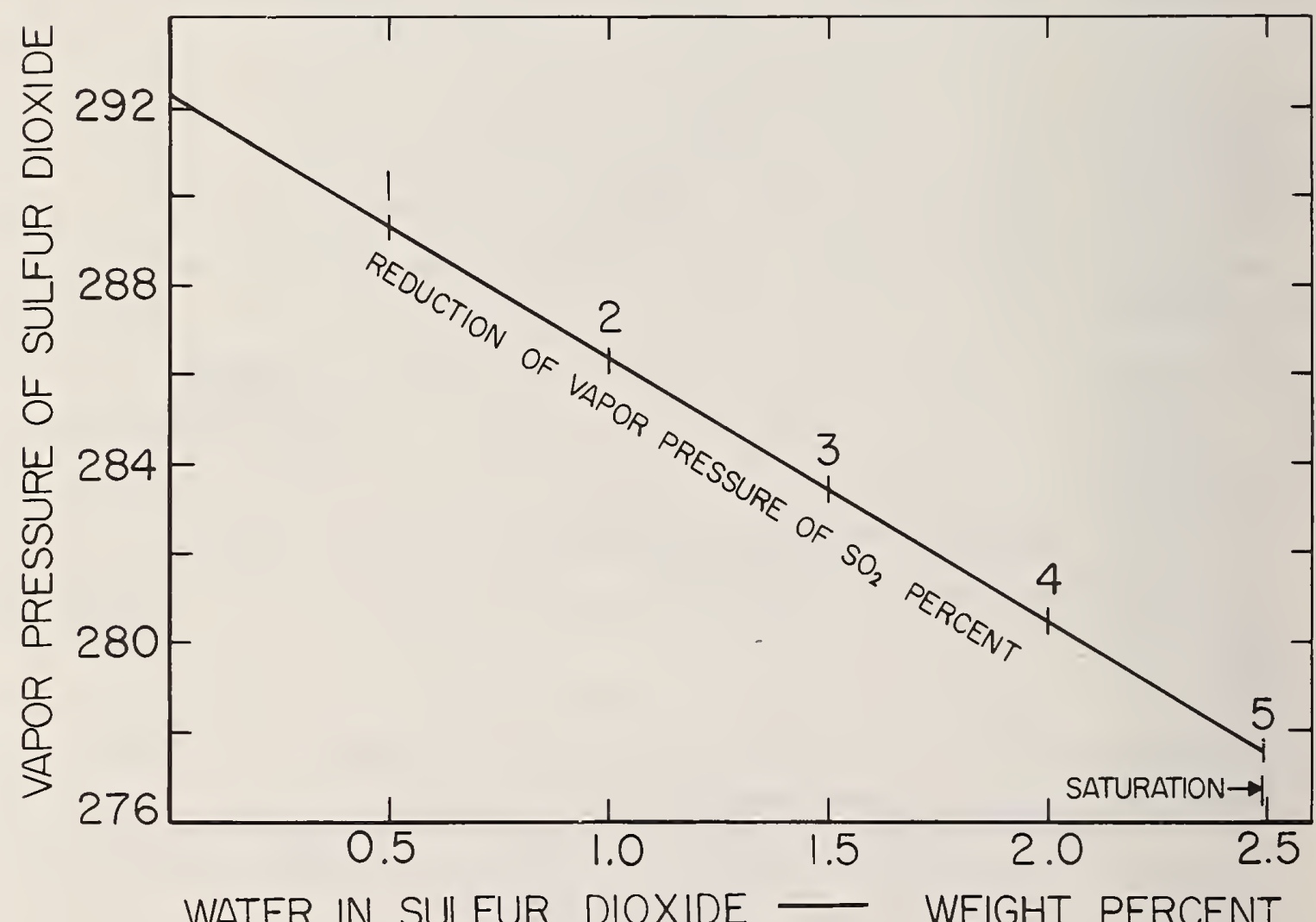

Figure 7. Predicted decrease of vapor pressure of $\mathrm{SO}_{2}$. 
the pressure of water vapor should not exceed about $2 \mathrm{~mm}$ and the total pressure can be considered as almost entirely due to sulfur dioxide. When the amount of water in the system increases, the vapor pressure of water will increase and will continue to increase until the absolute pressure of water vapor equals the pressure of water vapor in the ambient atmosphere. At $25^{\circ} \mathrm{C}$ and 25 percent relative humidity, this will be about $6 \mathrm{~mm}$. The system will never contain more than a small amount of water, whether it is saturated or not, and the vapor pressure of $\mathrm{SO}_{2}$ at saturation, $277.5 \mathrm{~mm}$, represents the minimum vapor pressure of $\mathrm{SO}_{2}$ and; consequently, the minimum effusion. If the assumption that the effusion rate is proportional to the pressure of the effusing substance is valid; then the error due to absorbed water will never exceed 5 percent and most likely, will be less than this during most of the life of a tube. If tubes are continually exposed to air saturated with water vapor at room temperature, about $25 \mathrm{~mm}$ water vapor pressure, the rate of absorption of water will be increased and a reduction of the effusion rate of 5 percent of the original value will occur sooner than at 25 percent relative humidity, but no matter what the rate of absorption the minimum effusion rate should remain the same during the life of a tube while a sulfur dioxide phase still exists.

The simplest way to avoid this error is to minimize exposure of tubes to atmospheres containing large quantities of water vapor. Storage at low temperature, even at 100 percent relative humidity, would reduce the transfer of water as would volume. During these studies, several tubes handled with reasonable care have reached the point where no liquid phase was present. As long as even a discernible amount of liquid was present, the rate of effusion of sulfur dioxide remained the same as when the tube was first measured. An example is the results obtained for a single tube where a column of sulfur dioxide of $2 \mathrm{~mm}$ remained as compared with an original of more than $90 \mathrm{~mm}$. The liquid was yellow in color, consisted of a single liquid phase, and con- 
tained a small residue of particles resembling flakes of rust. The effusion rate at $25^{\circ} \mathrm{C}$ determined while the tube contained more than 50 percent of liquid was 2.766 micrograms per minute. The rate determined with only $2 \mathrm{~mm}$, or less than 3 percent of the original sulfur dioxide, was 2.764 micrograms per minute. The difference is considerably less than the approximate 1 percent uncertainty of measurement of the original rate and is considered to be insignificant. Accordingly, it is concluded that with only moderate care the effusion rate will not be seriously affected by exposure to the ambient atmosphere.

Another source of error which cannot be easily evaluated concerns the purity of the sulfur dioxide within the tube. An implicit assumption of the gravimetric calibration is that the contents of the tubes are pure and that the weight loss represents only the loss of sulfur dioxide. Measurements of several tubes identical in external dimensions but differing in permeation rate by 10 percent, using the instrumental technique previously mentioned, showed good correlation between the quantity of sulfur and the gravimetrically observed rates. These same tubes and one having an intermediate rate were sealed in evacuated glass ampoules and were allowed to effuse for a period of several days. The gases which accumulated were then examined mass spectrometrically. All consisted only of sulfur dioxide with no evidence whatever of any other species except small quantities of air in amounts normally observed as "background."

It is conceivable that the situation could arise where impure sulfur dioxide could be used in the fabrication of a lot of tubes. However, the quality control of commercial sulfur dioxide is believed to be good so that such an occurrence is unlikely.

b. Results. The effusion rates of 160 tubes were determined gravimetrically during this study. The tubes were produced by two different manufacturers presumably from different supplies of tubing and had slightly different design 
characteristics. Tubes from one source, hereafter referred to as "A" were in general of the same wall thickness but of different lengths. Tubes from the other source, "B," were all of the same length but of different wall thickness. It was observed early in the study that tubes from source "B" were in general more erratic in behavior than those from source "A." This may be due in part to the use of a thicker-walled Teflon tubing but as yet the cause is not entirely identified. In the final calculation, some of the tubes from "B" were not included because their behavior was not considered typical in terms of the intent of the study.

All of the gravimetric data for the measurements at various temperatures was computer processed. The equation

$$
\log \mathrm{R}=\mathrm{mT}+\mathrm{b}
$$

where $R$ is the rate in micrograms per minute and $T$ is the temperature in degrees absolute, was found to give a straight line in the temperature interval between 20 and $30{ }^{\circ} \mathrm{C}$. The data were fitted by a method of least squares and the constants $m$ and $b$ as well as the error associated with them were calculated. The rates at 20,25 and $30^{\circ} \mathrm{C}$ were then calculated as was the uncertainty in the rate at each temperature.

The voluminous results are not reproduced here but several points of interest were noted and will be expanded upon.

The calibration uncertainty associated with two different groups of tubes was found to be essentially the same even though one was based upon as many as 51 useable weighings while the other was based on only 7. Although the second calibration was done at a later date and involved minor refinements of techniques it indicates that an adequate degree of accuracy in determination of the rate can be achieved in a relatively short period of time without loss of a significant amount of material from the tube. Table 1 lists the rate at $25^{\circ} \mathrm{C}$ for several tubes together with the uncertainty (expressed as 
the 95 percent confidence limit of the average) and the number of useable weighings. A useable weighing is simply a weight obtained after the tube has reached equilibrium at a particular temperature. Since hysteresis does occur, weights obtained shortly after a temperature change probably do not reflect the equilibrium rate of weight loss. No significant difference in the uncertainty appears between the two groups. It can be concluded that an uncertainty of about \pm 2 percent would constitute a conservative limit to be placed on values obtained by the gravimetric technique.

Table 1. Reliability of gravimetric calibration.

\begin{tabular}{cccc}
$\begin{array}{c}\text { Tube } \\
\text { number }\end{array}$ & $\begin{array}{c}\text { Rate in } \\
\text { micrograms } \\
\text { per minute }\end{array}$ & $\begin{array}{c}\text { No. of } \\
\text { determinations }\end{array}$ & $\begin{array}{c}\text { Relative error, } \\
95 \% \\
\text { confidence } \\
\text { limit }\end{array}$ \\
\cline { 2 - 3 } & 1.321 & 7 & 1.92 \\
6231 & 2.52 & 9 & 1.48 \\
6229 & 2.57 & 9 & 1.55 \\
6235 & 2.67 & 9 & 1.15 \\
217 & 1.35 & 48 & 2.09 \\
137 & 2.67 & 45 & 1.19 \\
157 & 2.71 & 44 & 1.60 \\
128 & 2.92 & 48 & 1.20
\end{tabular}

Another significant point is the larger uncertainty associated with tubes from source "B." Reproduced in Table 2 are the errors associated with 20 tubes selected at random from batch "A" and "B." The average as well as most individual results show a significant difference.

The tubes from source "B" differ little from "A" except in external diameter and in wall thickness. The larger wall thickness, as previously mentioned, is believed to be the source of this larger error. A theoretical treatment of the mechanism of diffusion through a porous membrane would perhaps 
confirm that a greater uncertainty would be experienced when thicker membranes are used. For the present, however, let us examine the process in an empirical manner simply to allow some speculation as to what might be occurring. It was observed when these tubes were first investigated that permeation rates

Table 2. Typical calibration uncertainties.

Tube

number

1

2

3

4

5

6

7

8

9

10

\section{Source "A"}

1.56

1.59

2. 24

2.92

1.66

1.80

0.87

1.23

2.10

1.89

1.78
Source "B"

2.72

4.49

5.83

3.02

3.10

5.22

2. 54

3.75

3.72

3.00

3.74

Average:

of tubes from "A" were more or less independent of the rate at which air passed over them. Tubes in an exposure chamber to which the air supply was inadvertently reduced or shut off were found to have about the same permeation rate as when a moderate stream of air was flowing. Tubes from "B," however, exhibited generally different behavior in that very erratic permeation rates were observed when the air flow through the chamber was reduced. The results obtained on successive days for one tube from each batch exposed at low or zero air flow rates are shown in Table 3. (The average for the tube from "A" was the same in an adequate flow of air.) The average permeation rate for the tube from "B" determined while an adequate flow of air was maintained was found to be $1.37 \mathrm{mg} / \mathrm{min}$ 
or about the same as the average of the erratic set. This suggests a conservation of sulfur dioxide, and that in spite of the erratic loss from the outer surface, the mass flow of sulfur dioxide across the inner surface remains constant. A thicker wall must of necessity have a lower concentration gradient expecially near the outer surface. Perhaps the slope of the gradient for a thick wall is so low that variation in sulfur dioxide concentration in the boundary layer caused by variation in the rate of removal of sulfur dioxide can cause a temporary reduction and even reversal of the rate of loss from the surface.

Table 3. Storage behavior.

Determination

No.

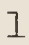

2

3

4

5

6

7

8 $\frac{\text { Rate in micrograms per minute }}{\text { "A" }}$

0.96

2.18

0.97

1.20

0.94

1.31

0.94

1.46

0.96

1.22

0.96

.54

1.61

0.96

c. Storage and Use. A tube which originally contains about a gram of sulfur dioxide and which loses sulfur dioxide at a rate of 2 $\mathrm{gg}$ per minute at room temperature will have a useful life of less than one year. Therefore, if permeation tubés are to have maximum service life it is necessary to reduce the effusion rates when the tubes are not in use. A simple procedure is storage at low temperature with removal only when needed for calibration purposes. It has been observed, however, that there is considerable lag in response to temperature changes. It is extremely difficult to observe 
this hysteresis quantitatively over a short period of time. Three tubes which had been stored at temperatures between -20 and $-30{ }^{\circ} \mathrm{C}$ were compared with a tube of known rate at various times after removal from the low temperature. The results are shown in Table 4. The rates are the observed rate as a percentage of the final rate measured 72 hours later. The readings at 24,48 and 72 hours are considered constant within the limits of the method. The readings after 3 and 6 hours definitely indicate that equilibrium has not been reestablished.

\section{Table 4. Recovery of rate after removal from low temperature storage.}

Time after removal/hours

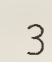

6

24

48

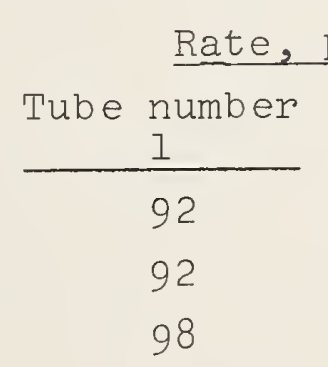

100

\section{Rate, percent of final value}

\begin{tabular}{cc} 
Tube number & Tube number \\
\hline 92 & $\frac{3}{91}$ \\
92 & 91 \\
99 & 100 \\
100 & 100
\end{tabular}

Because of the basic difficulty in following small changes in effusion rate with the instrumental technique an attempt was made to determine the rate of change of the rate after storage at low temperature by a gravimetric technique. Several tubes were removed from low temperature storage $\left(<-30^{\circ} \mathrm{C}\right)$ and were weighed as often as practical during a 24 hour period after removal. The rates at the various times were then calculated. The results were quite erratic and effusion rates calculated from the weight losses were inconsistent. The probable cause of the inconsistency is the error associated with the determination of effusion rates using short term weight losses. Accordingly, a single tube was removed from low temperature storage and suspended from the balance. The weight of the tube was then determined over a period of 36 hours without removal from the balance. The 
observed temperature in the balance case fluctuated about \pm 0.1 degrees but was not significantly different from the average at any time during the 36 hours. Experience has shown that the magnitude of the long term drift of the balance zero would have no significant effect on the overall rates described below. Weights were determined at intervals varying from 10 minutes to 2 hours except during the overnight period. Two different rates were observed. These are shown in figure 8. The slope of the two indicated portions of the curve were computed and their intercept was calculated. The results indicate that the intercept would have occurred 886 minutes after removal from cold storage. The effusion rate before the intercept is only 86 percent of the true rate observed after equilibrium had finally been reached.

The above results confirm the earlier and less exact work using the flame photometric technique. However, the question as to the minimum waiting period after removal from storage is still unanswered. If the transition from one rate to the other was abrupt the minimum time would be the time of intercept. If the intercept is only theoretical and the transition is in fact gradual then the minimum waiting period would be greater than the intercept would indicate. Accordingly, a second tube was removed from low temperature and the weight loss was recorded in the same manner. Again, different slopes were observed over a period of 32 hours. The calculated intercept for this tube was 993 minutes. However, a change in slope of the line representing the lower rate began to occur after approximately 700 minutes. The slope, in the region from 700 through 800 was about $2.44 \mu \mathrm{g} / \mathrm{min}$ determined graphically. This value is intermediate between the early rate and the final rate. If a symetrical transition is assumed then the minimum waiting period would be about 1300 minutes. Since it is known that 24 hours (1440 minutes) is sufficient to reestablish the time rate it is recommended that this latter value be used as a safe minimum waiting period. 




Figure 8. Change in weight of permeation tube after low-temperature storage.

Teflon is known to experience a double phase transition in the region between 0 and $40^{\circ} \mathrm{C}$ with the transitions centered around 20 and $30^{\circ} \mathrm{C}$ [9]. Equilibrium is not rapidly reestablished and the experimental data indicate that temperature changes of one degree per hour are not sufficiently slow to 
maintain equilibrium. Considerable periods of time may be required to reestablish the steady state specific volume of Teflon depending on the rate of change of temperature and the magnitude of the change. It is not known whether these phase changes will have a major effect on the effusion rate but the effect is highly suspect as a reason for the hysteresis observed when temperature cycling of the tubes occurs. It was consistently observed when determining the rate of effusion of the tube that the first rate determined after a change of temperature usually differed from the succeeding rates and the difference was always in the direction of the previous temperature. As an illustration, the results obtained with a single tube are shown in Table 5. The behavior for this tube is quite typical for other tubes in the same set treated in the same manner.

The values are the condensed results in the order in which the temperature was changed. The initial reading appears at the indicated temperature and the final (approximately the average) rate obtained after a series of measurements at the indicated temperature appears immediately below.

Table 5. Temperature cycling behavior.

Temperature, ${ }^{\circ} \mathrm{C}$

26.3 (final)

29.4 (initial)

29.4 (final)

20.0 (initial)

20.0 (final)

31.0 (initial)

31.0 (final)

25.5 (initial)

25.5 (final)

30.4 (initial)

30.4 (final)
Measured rate, $\mu \mathrm{g} / \mathrm{min}$

3.09

3.80

3.86

2.23

1.85

2.85

4.47

4.89

2.86

3.21

4.23 
The only change not characterized by a difference from the final value is the transition from 31.0 to $25.5{ }^{\circ} \mathrm{C}$. This, however, occurred over a longer period of time and equilibrium had probably been well established before the first weighing was made.

The period of time represented by the interval between temperature changes was at least one day. The first rate at the new temperature was always based on a rate determined after the temperature change occurred and was not based on a weight loss measured between different temperatures.

The calibration procedure requires equilibration at the temperature of calibration for long periods of time. The time is presumably more than sufficient to reestablish equilibrium in the Teflon. However, in use the tubes may be used at different temperatures for short periods of time in order to produce different concentrations of sulfur dioxide in a gas stream. If, for example, a tube normally stored at room temperatures is used for a short time at $30^{\circ} \mathrm{C}$, what periods of time are involved in reequilibrating the tube? Several tubes were stored at $25^{\circ} \mathrm{C}$ and their rates relative to a reference were determined using the flame photometric instrument. They were then stored at $31{ }^{\circ} \mathrm{C}$ for periods varying from 135 to 290 minutes. The tubes were then immediately cooled to $25^{\circ} \mathrm{C}$ and the rate of loss of sulfur dioxide was periodically determined until the rate returned to the value previously obtained at $25^{\circ} \mathrm{C}$. The results are shown in Table 6. The length of storage at $31{ }^{\circ} \mathrm{C}$ is shown. The rate is indicated as the percent of the rate originally observed before storage at $31{ }^{\circ} \mathrm{C}$.

This experiment was repeated several times and from these results and other experiments it appears advisable that the tubes should be used at a single fixed temperature. If different temperatures are used, then sufficient time should be allowed for the reestablishment of equilibrium. The time 
will depend to a large extent on the length of time a tube has remained at a temperature differing from that to be used. In general, tubes stored or used at one temperature for periods greater than one day should be allowed to equilibrate at any new temperature for at least 24 hours. Tubes cycled over short temperature ranges in short periods of time can probably be used at the starting temperature with little change in accuracy of the rate. If the temperature is changed permanently, the rate at the second temperature cannot be expected to reflect the true rate at that temperature within a period of time less than 24 hours.

Table 6. Short term temperature effects.

Tube number Time at $31^{\circ} \mathrm{C}$ Time at $25^{\circ} \mathrm{C}$ Percent of $25^{\circ}$ rate

\begin{tabular}{|c|c|c|}
\hline 171 & $135 \mathrm{~min}$. & $\begin{array}{l}10 \\
25 \\
43 \\
67 \\
87\end{array}$ \\
\hline 157 & $230 \mathrm{~min}$. & $\begin{array}{r}2 \\
7 \\
11\end{array}$ \\
\hline 146 & $290 \mathrm{~min}$. & $\begin{array}{l}11 \\
40 \\
45 \\
65\end{array}$ \\
\hline
\end{tabular}

Another interesting detail gleaned from the tabulated results is shown in Figure 9. The rates are plotted with respect to tube number in ascending order. This is the only set of tubes in which it was known that the tubes were prepared from a continuous length of tubing and where the tube numbers indicate the position of the tube relative to the others. It is obvious from the regular variations that a significant difference in permeation rate is associated with different sections of the stock tubing from which the tubes were prepared. 


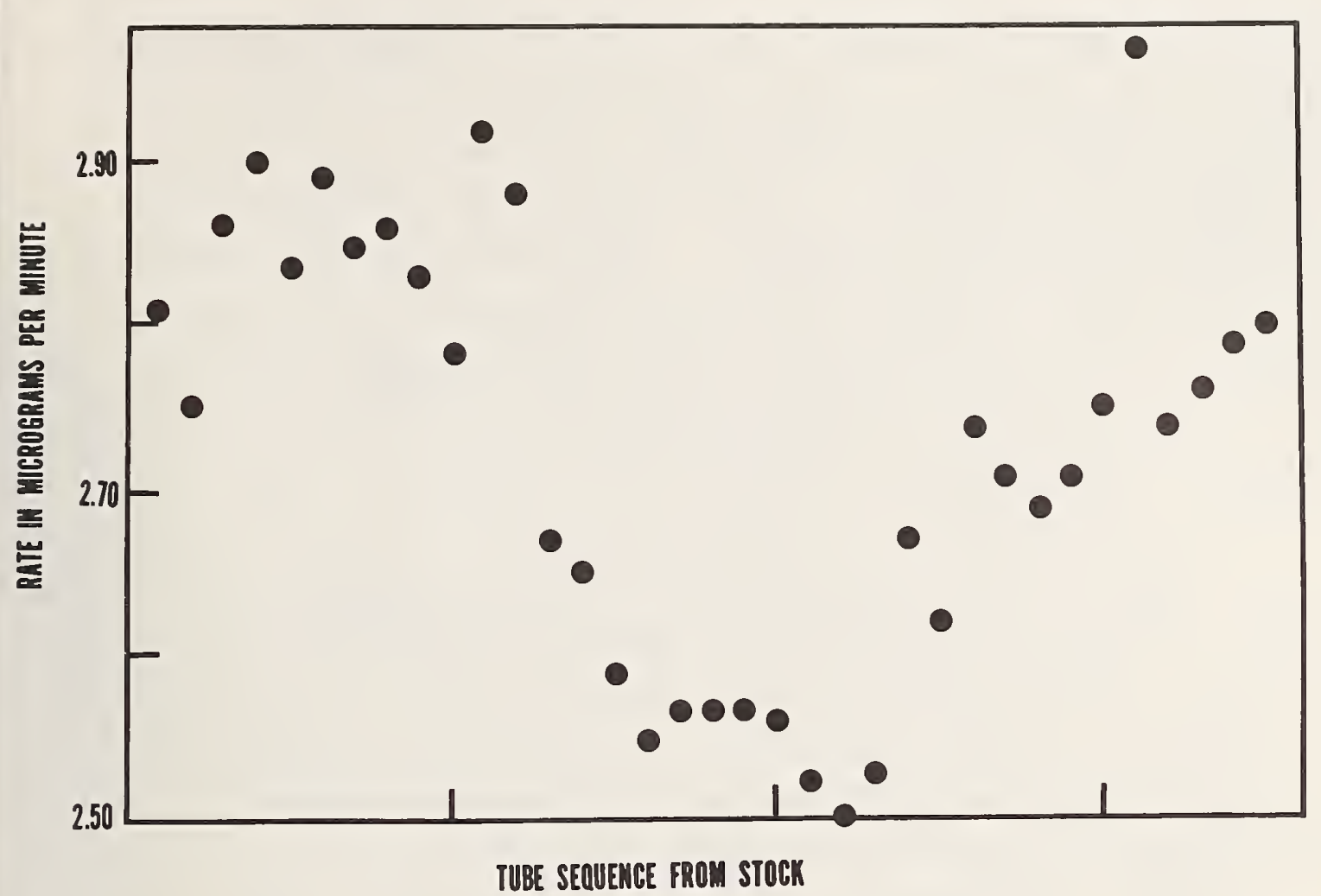

Figure 9. Correlation of permeation rate with tube sequence.

d. Conclusions. Sulfur dioxide permeation tubes provide a practical means of producing gases of accurately known concentration of this substance for calibration of air pollution measurements. The disadvantages of stringent requirements for accurate temperature control and measurement as well as an accurate knowledge of flow rate of ambient air are offset by the inherent reliability of the gaseous mixtures that can be so produced. Accordingly, the tubes seem to be superior to any other means of producing a gas of known $\mathrm{SO}_{2}$ content。

The studies described above have demonstrated the feasibility of certification of permeation tubes as SRMs for air pollution analysis and a program for this purpose has been initiated. Plans call for certification of commercially 
produced tubes on the basis of individual calibration. The gravimetric procedure already described will be used. In addition, tubes selected from each lot will be checked by coulometric calibration [3] and also by measurements with the flame photometric detector. Mass spectrometric measurements will also be made of the effused gas to verify its composition. A certificate giving the measured permeation rates of each tube at several temperatures will be furnished. This SRM is expected to be available for distribution in latter part of 1970 .

In connection with this certification program, NBS will maintain a group of permeation tubes as NBS Laboratory Standards. These tubes will be periodically recalibrated and intercompared. Tubes certified for public use as SRMs will be compared with the NBS Laboratory Standards as part of the calibration procedure already described.

The NBS SRM permeation tubes will provide a means not only to calibrate analtical instrumentation but will also make possible the interlaboratory comparison of techniques and methodology. Furthermore, their use as laboratory controls would virtually eliminate measurement uncertainty as a factor in questions concerning ambient air quality.

\section{Organic Materials}

A number of organic standard reference materials have been examined in the past year. While not gaseous materials under ordinary conditions, these standards were amenable to investigation by mass spectrometry and hence were analyzed by this laboratory. Much of this work was concerned with the examination of material concerned with the clinical standards program。Bilirubin, cholesterol, urea, and various derivatives of substances related to these materials were examined. The entire NBS line of microchemical standards was also examined, since they have been shelf items for several years and their stability needed verification (see section $3 . B .2$ of this report)。 
C. High-Purity Gases

A frequent problem in gas analysis is the determination of the purity of commercially produced gases. Probably the most convenient and potentially most informative instrumental technique is mass spectrometry. In general, samples can be analyzed with little difficulty and both the nature and quantity of many impurities can be readily determined. This is most often true for the less commonly encountered gases, and unfortunately, is least true for the common gases, oxygen and carbon dioxide. The major difficulty in the assay of oxygen is that sample pressure which would allow a simple and direct analysis of gases such as nitrogen, argon, helium and carbon monoxide are prohibitively high in the case of oxygen. Not only are compounds foreign to the sample formed in the ionization region at high pressures of oxygen but the oxygen itself has a very deleterious effect on the electron emitting filament of the instrument. In the case of carbon dioxide, the major impurity is nitrogen which has a mass approximately equal to that of the major fragment ion of carbon dioxide. Because of the great difference in concentration between the ion produced by the carbon dioxide and nitrogen high resolution mass spectrometry offers little analytical aid.

In the case of both these gases, a reagent is available which "selectively" removes the major constituent enabling a direct examination of the impurity. Oxygen can be completely removed with either phosphorous or a sodium-potassium alloy without removing nitrogen or the rare gases which are the normal impurities in reagent grade oxygen. Carbon dioxide, of course, can be removed with "Ascarite."

Two simple devices were constructed to facilitate these analyses. The first, shown in Figure 10, is designed for oxygen. The bulb marked "A" is filled with the sample and is then connected at "A." The bulb marked "B" contains the reagent. Preparation and use of the reagent, either phosphorous or sodium-potassium alloy is described in detail 
elsewhere [10]. The entire apparatus can be evacuated through "C" which is connected directly to the inlet system of the mass spectrometer. The sample is bled slowly into the reagent flask until the reaction is complete and a stable pressure minimum is reached as indicated by the manometer. If sodiumpotassium alloy is used, the sample may then be introduced directly into the inlet system and a mass spectrum is obtained.

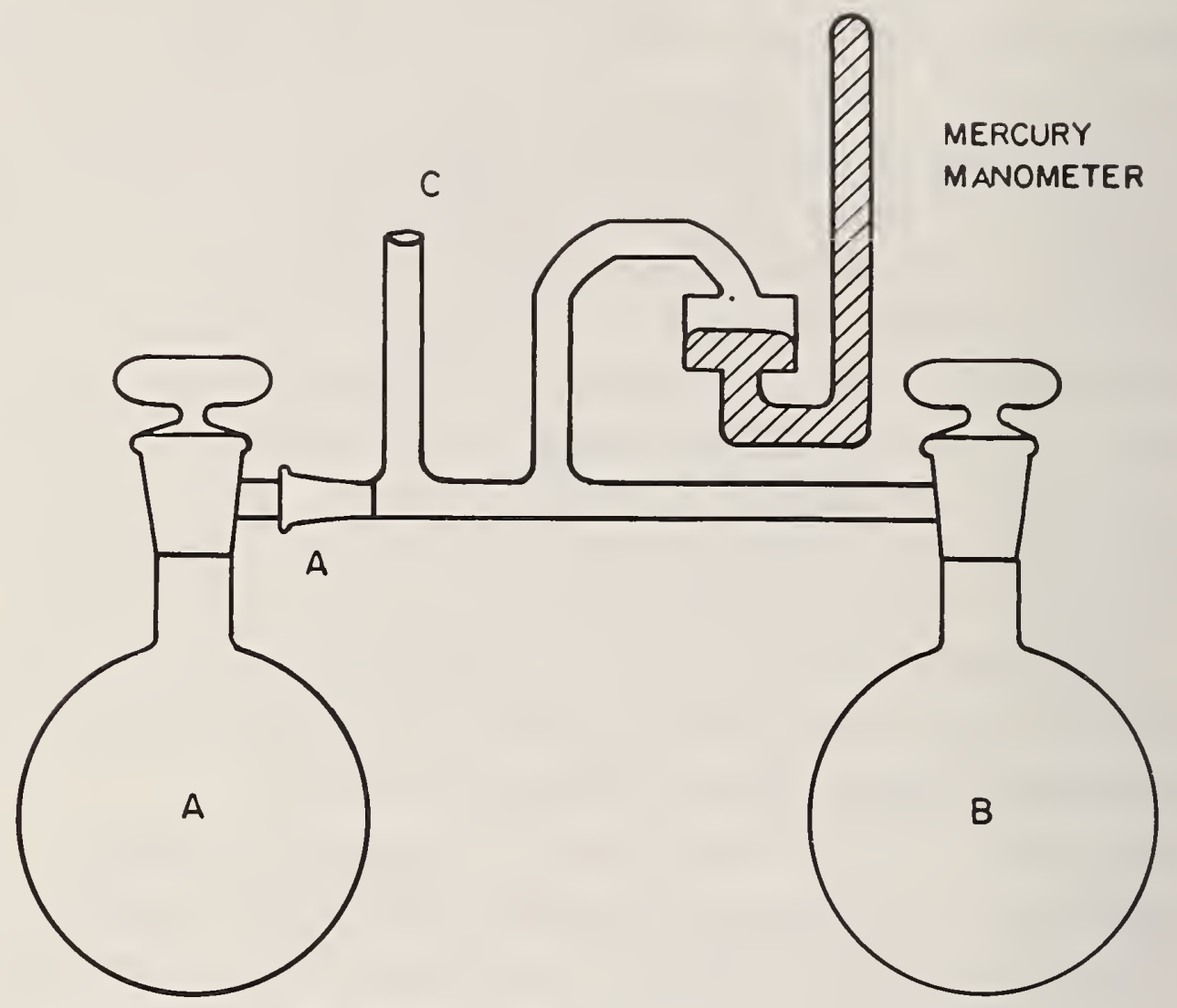

Figure 10. Apparatus for absorption of major constituent in a gas mixture.

When phosphorous is used, it is desirable to cool the bulb with dry ice after reaction to reduce the vapor pressure of phosphorous below the level where it interferes with the analysis. The impurities can then be expanded into the inlet system.

Some precautions should be observed by the careful analyst if reliable results are to be obtained. The metal alloy reacts 
readily with carbon dioxide and this gas even though present in the oxygen will not be observed in the residue. Too rapid a reaction may introduce spurious species through reaction with melted stopcock grease and possibly between the constituents of the sample. To minimize these problems, bulbs can be cooled either with a stream of air or in an ice bath. And lastly, both reagents are quite reactive and should be handled cautiously.

Experience has shown that more than 99 percent of the sample reacts in a reasonable length of time leaving the impurities together with less than 1 percent of the oxygen in the sample flask. The volume of the reaction flask and the inlet system can be readily determined allowing a simple calculation to be made of the quantities of the various impurities originally present in the oxygen. As an example the following was found in a sample of high-purity oxygen:

Nitrogen

Carbon Dioxide

Argon

Krypton

Xenon
133 parts per million by volume 35 parts per million by volume 8 parts per million by volume 16 parts per million by volume 3 parts per million by volume

The sample was analyzed using each reagent in order to avoid the possibility of overlooking an impurity which might react with one but not the other reagent. The pressure and identification of the rare gases is both plausible and certain as this oxygen was prepared by fractional distillation of air.

The apparatus for carbon dioxide shown in Figure 11 is simply a modification of a type of reagent tube long used in the field of gas analysis [11]. The sample flask "A" is filled with carbon dioxide from the cylinder to be analyzed. The flask is then attached at "A" and the entire apparatus is evacuated to the stopcock of the flask. The appropriate stopcocks are then opened or closed and the sample is allowed 
to pass slowly through the reagent column and then directly into the inlet system of the mass spectrometer. The magnesium perchlorate column is necessary to remove excessive water desorbed from the Ascarite. It has been observed that when using this technique, removal of the carbon dioxide may be incomplete if the sample is passed too rapidly through the reagent. This does not pose a serious problem, however, because it is only necessary to lower the carbon dioxide pressure to a value where the fragment ions are sufficiently reduced to allow a recognition and calculation of contributions from other species. Again, as with the oxygen, a determination of the ratio of the volume of the flask to that of the inlet system allows a simple calculation of the quantity of impurity.

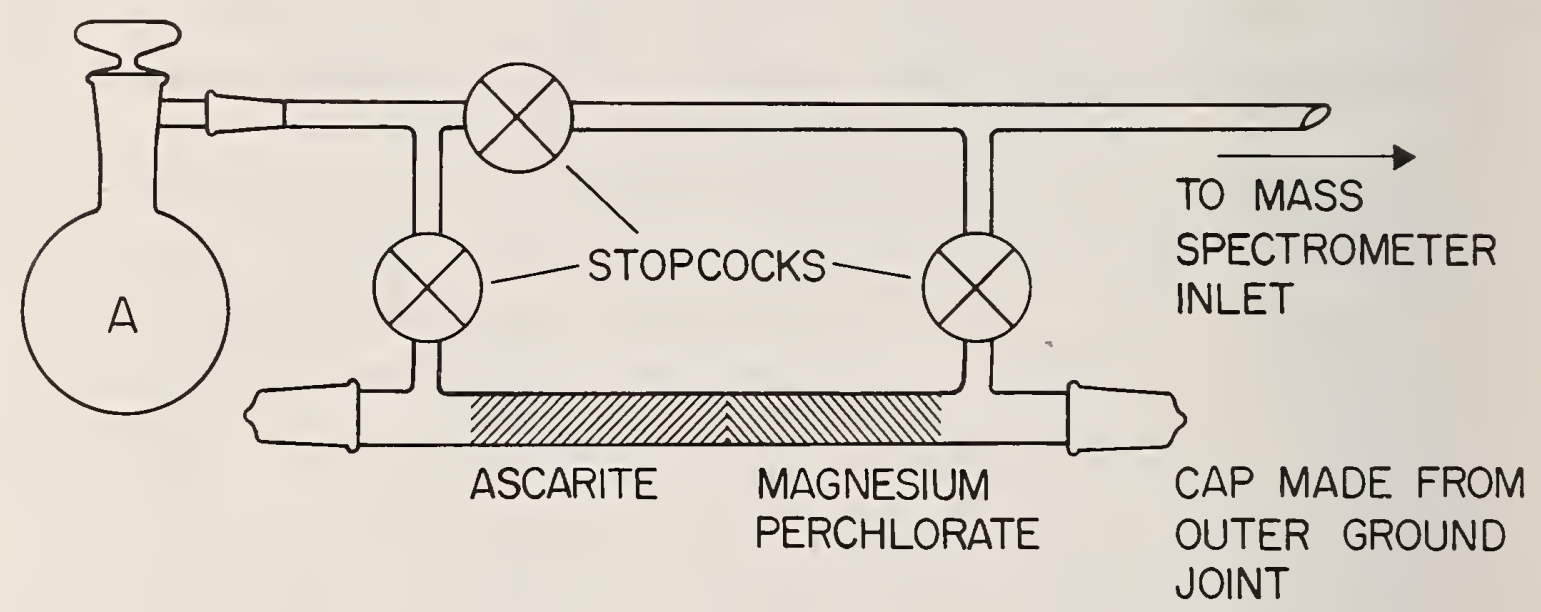

Figure 11. Device for concentrating impurities in carbon dioxide.

A simple device for transferring samples from the cylinder to the sample flask is shown in Figure 12. This device has been in use for several years and was inherited by one of the authors (E. E. Hughes) from predecessors in the former Gas Chemistry Section of the National Bureau of Standards. We have failed to note such an apparatus in the literature. We do not wish to claim any credit for its design but wish to pass it along as a useful device for transferring samples from cylinders, 


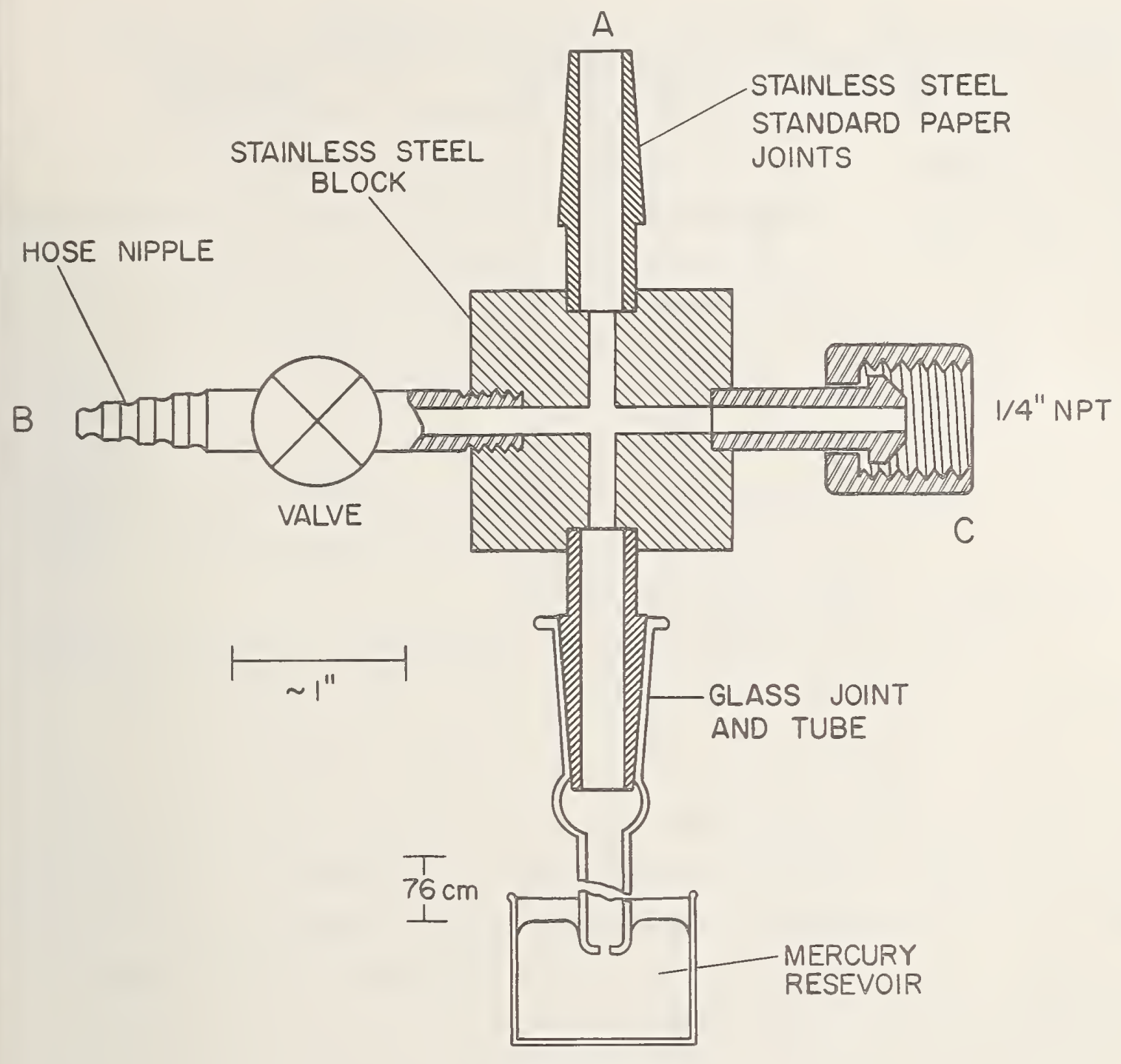

Figure 12. Device for minimizing contamination when sampling from gas cylinder.

with little or no danger of contamination from the atmosphere. When analyzing gases such as these described here, this latter source of contamination is of great importance and constitutes the single most significant source of error.

The transfer apparatus is connected directly to a control valve, not a regulator, attached to the cylinder. The previously evacuated sample flask is attached to the stainless steel 
ground joint (A). A moderate vacuum is applied through the valve (B). The cylinder valve $B$ is then closed and the cylinder control valve is opened slightly to allow gas to flow into the system. When the pressure reaches atmospheric as shown by escape of gas through the mercury reservoir, the sample flask may be opened and the pressure adjusted to one atmosphere. A series of evacuations and flushings prior to sampling gives the analyst a degree of confidence in the integrity of the sample that is found in few other techniques. The fact that the system need never be below a pressure of one atmosphere after the initial filling and flushing eliminates many sources of error such as small leaks in joints and around valve stems.

D. Atmospheric Oxygen Determination

A systematic determination of atmospheric oxygen has been described in previous reports [12] [13]. The results have been consolidated and have been published together with a discussion of their significance in relation to earlier investigations [14]. During the past year, several additional samples were obtained in a rural area in Maryland. The oxygen content of these samples was determined by comparison with a standard reference material (SRM 1609, Oxygen in Nitrogen). The results are shown in Table 7 .

The average agrees with that of the previous survey. Samples of a similar nature will be analyzed periodically in the future to establish a "benchmark" value for the present oxygen content of the atmosphere.

E. Research Support Services

This laboratory analyzes a wide variety of gaseous samples related to the research programs of other NBS divisions as well as samples for other government laboratories. Carefully compounded and analyzed gaseous mixtures are also prepared on occasion. The analytical services mainly utilize mass spectrometry but other techniques, including gas chromatography and electrochemistry are used as required. 
Table 7. Atmospheric oxygen determination.

Sample No.

1

2

3

4

5

6

7

8

9

10
Oxygen content in mole \%

20.944

20.949

20.946

20.946

20.947

20.944

20.949

20.945

20.946

20.947

20.946

Gravimetric procedures are used to compound gaseous mixtures, provided the overall mass, including container, does not exceed $10 \mathrm{~kg}$ 。 Otherwise, pressure measurements with carefully calibrated gauges are used to compound mixtures.

Gas analysis ranges in complexity from simple identification of specified substances to the quantitative determinations of trace or residual components in highly complex mixtures, such as reaction products. Many of these are done on a continuing basis and are scheduled to coordinate with experimental work in progress in other laboratories. Table 9 lists the more significant analyses during the past year. Several of the more interesting items are below.

1. Products from Polymer Degradation Studies

This laboratory cooperates with the NBS Polymer Division in the analysis of the gaseous and liquid products of thermally degraded polymers. A total of 18 samples were received over a six month period. The samples were contained in glass 
ampoules and mass spectrometry was used to determine the total pressure as well as to identify and/or quantitatively determine the gaseous products.

The determination of thermal degradation products of polyimides is given as an example of this kind of analysis. Four $50 \mathrm{ml}$ ampoules, designated "A," "B," "A Iiq," and "B Iiq" were received. The gas pressure in both "A" and "B" was $2 \mathrm{~mm}$ Hg. The pressure in the remaining two ampoules was not of interest so was not measured. The compositions of these samples are given in Table 8.

Table 8. Gaseous products from thermally degraded H-film. Composition in mole percent

Component
$\mathrm{N}_{2}$
$\mathrm{CO}_{2}$
$\mathrm{H}_{2}$
$\mathrm{O}_{2}$
$\mathrm{H}_{2} \mathrm{O}$
$\mathrm{CO}$

\begin{tabular}{|c|}
\hline "A" \\
\hline 1.5 \\
\hline 1.2 \\
\hline 13.5 \\
\hline 0.7 \\
\hline 83.1 \\
\hline
\end{tabular}

Benzene

Xylenes

HCN

0.9

Trace

2.5 $\frac{\text { "B liq" }}{1.4}$

90.8

4.1

0.9

2.8

Other samples contained such components as $\mathrm{N}$, N-dimethyl acetamide, benzonitrile, phenol, aniline, and the fluorocarbons $\mathrm{C}_{2} \mathrm{~F}_{4}$ and $\mathrm{C}_{3} \mathrm{~F}_{6}$. Most contained water and air in various amounts.

2. Helium-3 Analysis

In a continuing program in cooperation with the Monsanto Research Corporation, this laboratory periodically analyzes a series of $\mathrm{He}-3$ samples for He-4 and other impurities. The He-3 samples have been taken from bulk lots of $\mathrm{He}-3$ which are produced for use in gas thermometry. The analysis performed by this laboratory constitutes NBS certification of the He-4 content of the $\mathrm{He}-3$. 
The He-4 content of the samples ranges from about 0.007 to 0.01 mole percent. In order to analyze for $\mathrm{He}-4$ at this low concentration, "high pressure mass spectrometry" is utilized, i.e., the sample pressure is about $10 \mathrm{~mm}$ as opposed to pressures of 0.01 to $.2 \mathrm{~mm}$ for normal operation. High gas pressures increase the signal of components present in small quantities, but are only feasible with gases which will not adversely affect the spectrometer or complicate the interpretation of the spectrum. The high pressure technique results in $\mathrm{He}-4$ analyses with a precision of about \pm 0.0005 mole percent at the above mentioned He-4 levels. During the year, a total of 10 samples of $\mathrm{He}-3$ were analyzed for $\mathrm{He}-4$, and 2 samples were analyzed for trace impurities such as water, nitrogen, oxygen, argon, and carbon dioxide. These impurities range from a few hundredths to a ten-thousandth of a mole percent which is about the limit of detection by this technique.

\section{Polywater.}

Attempts have been made to observe significant ions in the mass spectral pattern of several samples of "polywater" and related "poly-alcohols." Samples prepared by Dr. Warren Grant of the NBS Polymer Division were submitted either in the capillaries in which the material was prepared or on the surface of a diamond plate, after removal from the capillaries. Samples were analyzed with a double focusing mass spectrometer. A heated probe was used to insert the samples as close to the ionization region as possible.

Many problems were encountered which precluded definitive results [15]. The primary problem arose from the small quantities of material which were available with a resulting difficulty in distinguishing spectra arising from the sample from spectra generated withing the instrument. Accordingly, quantitative interpretation of the spectra was not possible and no further work is contemplated until larger samples are available. 
A. Mass Spectrometric Analysis

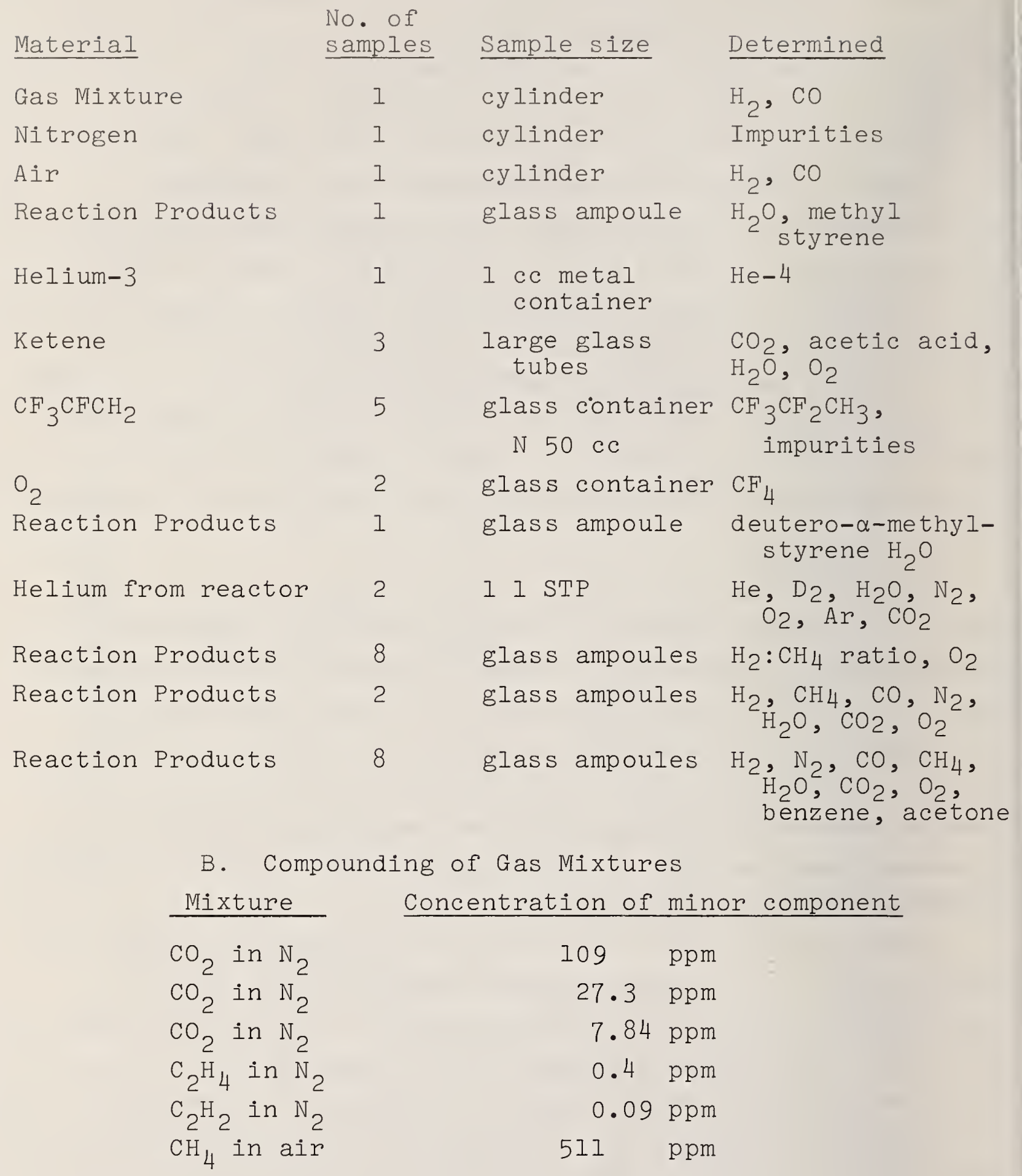

(E. E. Hughes, J. M. Ives, and G. Silver) 40 
A. Introduction

The activities of the Polarographic Laboratory have been concerned largely with the analysis of standard reference materials and with samples of research materials connected with the programs of other NBS sections as well as several other government agencies. Determinations of major constituents have been an important part of the program; however, the major emphasis has been on trace analysis. As the end of the reporting year approached, considerable effort was being devoted to environmental analysis, especially the determination of certain metallic elements in particulate samples. All of this was accompanied by a considerable amount of method development activity .

A recapitulation of the year's work shows about 15 major reports concerned with standard reference material analysis and about 10 reports on the analysis of research materials. The more significant aspects of these activities are summarized in the following sections of this chapter.

B. New Facilities

Contamination introduced from particulate material in the laboratory air is a potential danger in trace analysis. Since many chemical operations need to be carried out over an extended period of time, exposure to even relatively clean air can significantly increase the analytical blank in many instances. To improve this situation, an assembly of clean air hoods has been installed in the chemical laboratory. This consists of four modular class-100 laminar flow clean hoods to provide 8 square feet of clean working area and an additional 8 square feet of clean air fume hoods.

Modular units, each having a working area of two feet by two feet, provide filtered air in a downward laminar flow, at rates adjustable from 50 to 150 linear feet per minute. The pre-filter consists of washable, reusable polyurethane 
foam and the final filter is an HEPA-absolute type with aluminum separators, with reported efficiency of 99.97 percent in the removal of particles with diameters of 0.3 micrometers or greater. The side panels are of plexiglass and the cabinet of steel, covered with epoxy paint.

Two of these modules, together with a modified module, constitute the clean area shown in Figure 13. The two modules in the foreground provide 8 square feet of working space to perform chemical operations normally done in the open laboratory as well as a storage area for processed material. The third module has been modified to provide a clean fume hood.

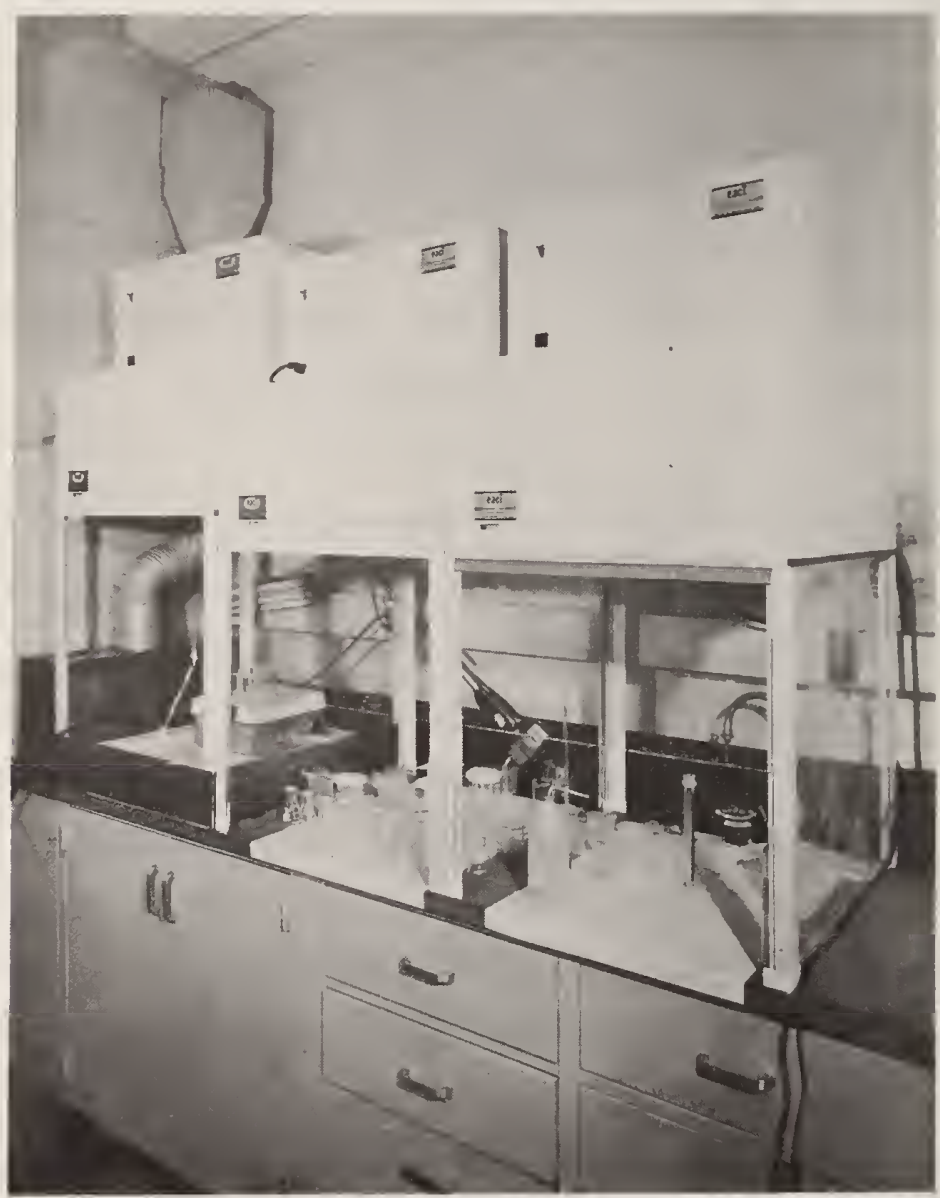

Figure 13. Three-unit clean air module. 
It is fitted with a perforated Teflon base and placed upon a fiberglass plenum which can be vented to the regular laboratory exhaust duct.

A different arrangement which also serves as a clean fume hood is shown in Figure 14. The vertical laminar flow hood is placed on a table in front of the conventional laboratory hood. The air from the clean unit is vented directly from its open front into the face of the laboratory hood.

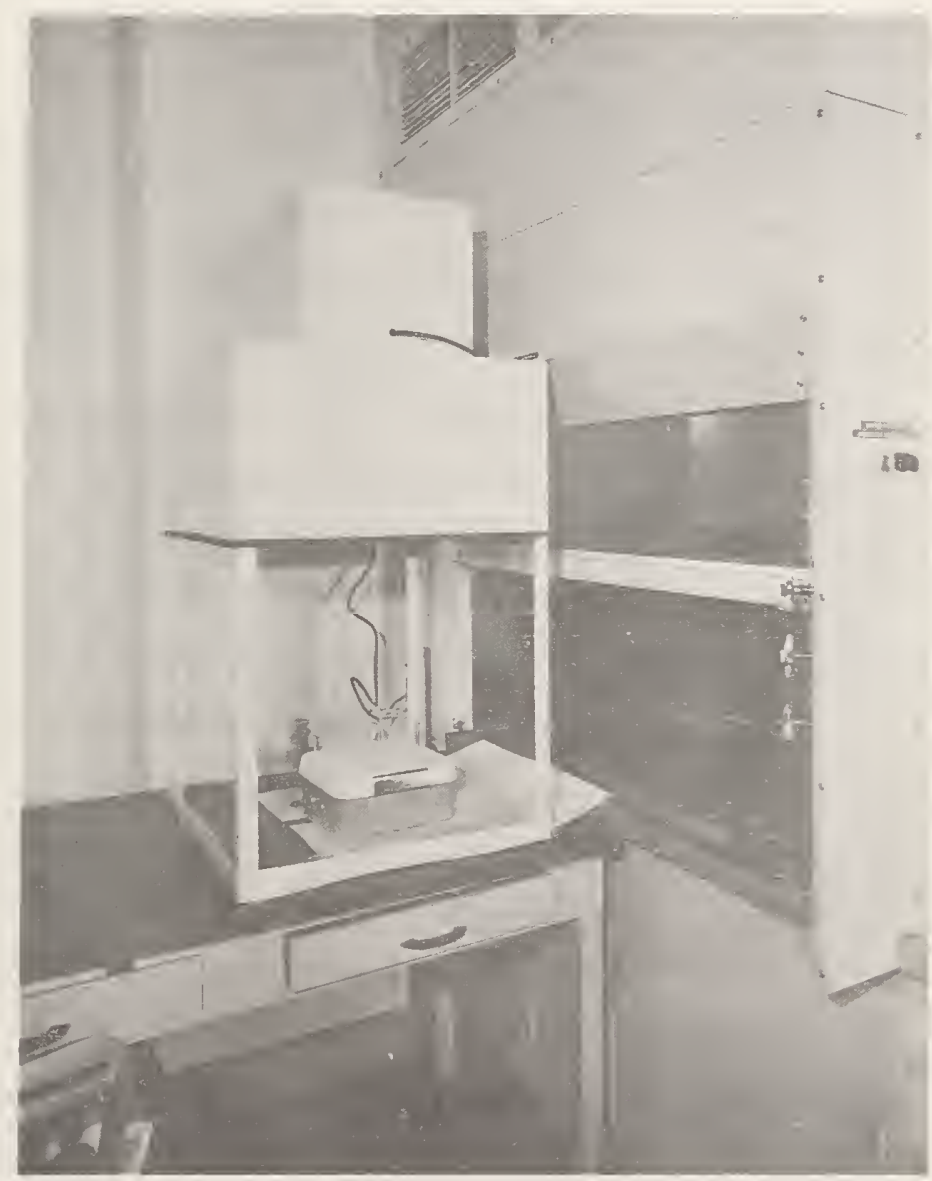

Figure 14. Single-unit clean air module.

The equipment described above has been in use for a period of about six months and is believed to provide considerable improvement over the exposure conditions prevalent in a 
relatively clean laboratory. Some experiments designed to evaluate the quality of the air within these hoods are described in Section F.2. on page 53 of this report.

C. Major Constituent Analysis

Both conventional and comparative polarographic methods have been under continuous development during the past several years to provide improved methods for microchemical analysis. The analysis of standard reference materials as well as research materials from other NBS programs has been facilitated. A few examples are described in the following sections.

1. Copper and Zinc in Metal Foils

A sample of metal foil, believed to be either $70 \mathrm{Cu}-30$ Zn or $80 \mathrm{Cu}-20 \mathrm{Zn}$ was submitted for identification. A 170-mg sample was dissolved in nitric acid and diluted to one liter with a supporting electrolyte of pyridine- pyridinium chloride. Copper and zinc were then measured concurrently at about -0.4 and $-1.1 \mathrm{~V}$, respectively Vs. a mercury pool anode by cathode ray polarography. Calibrations were established using SRM 45d, melting point copper, and SRM 43e, melting point zinc, respectively as standards. This particular analysis gave results of 80.4 percent $\mathrm{Cu}$ and 18.7 percent $\mathrm{Zn}$. While a relatively large sample was available, similar results could have been obtained on fractional milligram samples, by appropriately adjusting the volume of solution. Under such circumstances, weighing of the sample would be the major source of error.

2. Copper and Nickel in Foils

Several foils containing major concentrations of copper and nickel with iron as a minor constituent were submitted for analysis. These foils were to be used as standards for spectrographic analysis for which purpose the major constituents needed to be determined within 0.5 percent. Samples weighing 8, 10, and $23 \mathrm{mg}$, respectively, were dissolved and diluted to either 50 or $200 \mathrm{ml}$ with a pyridine-pyridinium chloride supporting electrolyte. The copper and nickel reduction peaks were 
measured concurrently at about -0.4 and $-0.8 \mathrm{~V}$, respectively, vs. a mercury pool anode. The results are given in Table 10.

Table 10. Analysis of Cu-Ni foils.

Sample

designation

P 33

P 48

P 60

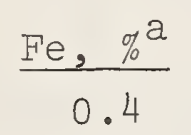

1.8

1.7

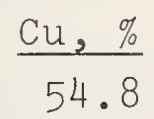

54.3

47.6

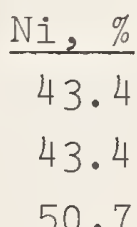

50.7 $\frac{\text { Total, \% }}{98.6}$

99.5

100.0

a By B. B. Bendigo, Analytical Coordination Chemistry Section.

3. Antimony in Lead-base Bearing Metal

A comparative polarographic method has been developed and used for the determination of antimony in lead-base bearing metal. The new method is equal in reliability but is more rapid than the long, conventional chemical procedure which involves a distillation, a hydrogen sulfide precipitation, and titration with potassium permanganate. A set of samples can easily be completed within several hours.

The procedure is as follows: One-gram samples are dissolved in dilute nitric and perchloric acids and heated to perchloric fumes. Concentrated hydrochloric acid is added, the solution is warmed gently to dissolve salts, and transferred to a l-liter volumetric flask. Ascorbic acid is added to reduce any iron present, and water is added to the flask to dilute to volume. Antimony is measured comparatively with respect to a suitable standard. In this case lead-base bearing metal, SRM 53e, served as the standard.

This method was used to determine antimony in a leadbase bearing metal standard undergoing certification analysis. This material is issued in chip form as SRM 53 e for chemical methods, and as SRM 1132 for use as a spectrographic standard. 
The SRM 53e had been completely analyzed by conventional chemical methods, as had all of the elements in the SRM 1132 except for the antimony content. Accordingly, SRM 53e was available as a standard for the comparative polarographic method, although previous members of the SRM 53 series could have been used.

The accuracy of the method is illustrated by the value of 10.22 percent antimony that was obtained. Due to the comparative nature of the method and availability of a reliable standard, this result is believed to be accurate to within 0.04 percent, absolute.

4. Cadmium in Cadmium Cyclohexanebutyrate (SRM 1053a)

The comparative polarographic technique has provided accurate results in the analysis and homogeneity testing of cadmium cyclohexanebutyrate (SRM 1053a) which is used for blending with lubricating oils to prepare standards for monitoring wear of engine parts. The following procedure was used: After drying over $\mathrm{P}_{2} \mathrm{O}_{5}$ for 48 hours, duplicate $0.4-\mathrm{g}$ samples were slowly ashed and ignited. The residues were dissolved in diluted hydrochloric acid and diluted to one liter. Cadmium was then determined polarographically at about $-0.7 \mathrm{~V}$ vs. a mercury pool anode using reference solutions prepared from cadmium metal which was approximately 99.99 percent pure. The results, as well as those later obtained by another technique are shown in Table 11 .

D. Minor Constituent Analysis

Previous experience in this laboratory has verified the sensitivity, selectivity, and accuracy of the polarographic determination of aluminum involving the reduction of the Solochrome Violet RS complex. Accordingly, procedures developed earlier for its determination in irons and stainless steels were modified and applied to the determination of aluminum in ferrosilicon, SRM 59a. 
Table 11. Cadmium in SRM 1053a.

Sample

designation

Top 1

Top 2

Top 3

Top 4
Cadmium, percent

Neutron activation

Polarography

analysisa

24.81

24.78

24.74

24.76

24.74

24.73

24.80

24.83

Average

24.77

24.81

ts

$\sqrt{\mathrm{n}} 95 \%$ confidence level 0.03

0.25

${ }^{a}$ By S. S. Nargolwalla and J. E. Suddueth, Activation Analysis Section.

The procedure is as follows: Samples of about $0.5 \mathrm{~g}$ were dissolved in nitric and hydrofluoric acids, fumed with perchloric acids and diluted to $50 \mathrm{ml}$. Five ml aliquots were taken and the iron was extracted with cupferron and chloroform. After adjustment of the $\mathrm{pH}$ to about 4.5 by addition of sodium acetate, the aluminum was extracted with chloroform and cupferron. Organic material was then destroyed with nitric and perchloric acids and aluminum was determined polarographically by reduction of the Solochrome Violet RS complex at about $-0.7 \mathrm{~V}$ vs. a mercury pool anode. The results are given in Table 12 along with those submitted by cooperators using other techniques. 
Table 12. Aluminum in ferrosilicon, SRM 59a.

Aluminum, percent

\begin{tabular}{|c|c|c|c|c|c|}
\hline Sample & Polarography & A & B & $\mathrm{C}$ & D \\
\hline $59 a, 1 B$ & 0.357 & & & & \\
\hline $59 a, 2 M$ & 0.344 & & & & \\
\hline $59 a, 3 M$ & 0.345 & & & & \\
\hline $59 a, 5 \mathrm{~T}$ & 0.345 & & & & \\
\hline $59 a, 5 B$ & 0.344 & & & & \\
\hline Average & 0.347 & 0.35 & 0.35 & 0.37 & 0.35 \\
\hline s & 0.006 & & & & \\
\hline
\end{tabular}

E. Small Sample Analysis

Work has continued on the analysis of very small samples of thin films vaporized onto a glass substrate. These films have been produced by the Harry Diamond Laboratory in the study of production of low temperature-coefficient circuitry materials for highly stable microminiaturized circuits. Such factors as resistive properties and the temperature coefficient of resistance need to be correlated with film composition. Since only several hundred micrograms of a sample is generally available, methods of high sensitivity and accuracy are required.

A number of samples were submitted for determination of copper, nickel, and chromium. In addition, nickel analyses were required on 3 tungsten filaments from which the metal film had been vaporized. The film was stripped from the plate with dilute hydrochloric acid and hydrogen peroxide and diluted to $50 \mathrm{ml}$. For chromium, 10-ml aliquots were taken and oxidized by heating with hydrogen peroxide and sodium hydroxide. The solutions were diluted to $10 \mathrm{ml}$ and the chromium reduction peak was measured at about $-0.7 \mathrm{~V}$ vs. a mercury pool anode. For copper and nickel, 10-ml aliquots were used. After reduction of chromium with $\mathrm{H}_{2} \mathrm{SO}_{3}$, the solutions were diluted to $10 \mathrm{ml}$ with a pyridine-pyridinium 
sulfate supporting electrolyte. The copper peak was measured at about $-0.5 \mathrm{~V}$ and nickel at about $-0.9 \mathrm{~V} \mathrm{Vs}$. a mercury pool anode. Values ranging from 8 to $12 \mu g$ of copper, 100 to $200 \mu g$ of nickel and 70 to $200 \mu g$ of chromium were obtained with $\mathrm{Ni} / \mathrm{Cu}$ ratios of about 25 and Ni/Cr ratios of about 1.5. Nickel in the tungsten filaments was determined in a similar supporting electrolyte after prolonged leaching of the filament. with hot nitric and hydrochloric acids. Seven hundred to 1000 micrograms of nickel was found on the filaments. F. Trace Analysis

Most of the effort in the past year in the polarographic laboratory has been concerned with trace analysis. The major problem in such analyses is the blank, arising from the environment as well as from the reagents used in the processing of the sample. Sufficient instrumental sensitivity is presently available and the techniques developed in this laboratory are capable of determining a nanogram or less of many elements, but the practical limit is often raised to the microgram level, because of the large blank involved. Attempts to alleviate this problem have been in two directions -- that of obtaining purer reagents, and that of providing a cleaner atmosphere during the period of chemical processing.

\section{Reagent Analysis}

A number of commercially prepared "high-purity" reagents have been supplied to us by the Separation and Purification Section. In order to evaluate their usefulness in certain determinations, it was necessary to first analyze them for the elements of interest.

a. Perchloric Acid. Perchloric acid of "highpurity" grade was available from two different manufacturers. One of the manufacturers also supplied two different lots prepared at different times. Iron, copper, lead, and cadmium were of interest and could be determined concurrently. 
Twenty-five or 30-ml portions of the different samples were evaporated to dryness in Teflon beakers. The residues were redissolved in $0.05 \mathrm{ml}$ of dilute hydrochloric acid, diluted with $5 \mathrm{ml}$ of an oxalic acid-ammonium oxalate supporting electrolyte, and measured subtractively. The results are shown in Table 13.

Table 13. Fe, $\mathrm{Cu}, \mathrm{Pb}$, and $\mathrm{Cd}$ in perchloric acid.

$\underline{\text { Sample }}$

Brand A

Brand $B$, Lot $X$

Brand $B$, Lot $Y$

\begin{tabular}{rccc}
\multicolumn{4}{c}{ Concentration, $\mathrm{ng} / \mathrm{g}$} \\
\hline$\frac{\mathrm{Fe}}{2}$ & $\frac{\mathrm{Cu}}{1}$ & $\frac{\mathrm{Pb}}{3}$ & $\frac{\mathrm{Cd}}{<1}$ \\
26 & 1 & 5 & 11 \\
18 & 1 & 3 & 18 \\
34 & 1 & 3 & $<1$
\end{tabular}

A method described by Pohl [16] for determination of a similar group of impurities in the $\mathrm{ppb}$ range in reactor water appeared to be applicable with some modifications to analysis of the acid. Pohl's method consists of addition of equivalent amounts of $\mathrm{Na}_{2}$ EDTA and cadmium solutions to the water sample. Those elements which form stronger complexes with EDTA than cadmium displace equivalent amounts of cadmium from the EDTA complex. The excess cadmium is then determined polarographically. The method was modified to operate at a $\mathrm{pH}$ of 3 for use in the analysis of perchloric acid. At a $\mathrm{pH}$ of 3 , metals reported to have stability constants higher than that of cadmium include copper, iron, mercury, nickel, and lead. Other metals which are reported to form more stable complexes than cadmium under the proper experimental conditions might also be included in this group. Some of these are vanadium, indium, thorium, scandium, gallium, zirconium, titanium, bismuth, zinc, and rare earths. The method as developed is as follows: Twenty-five or 30-ml portions of the different samples of perchloric acid were evaporated to 
dryness in Teflon. The residues were dissolved in $1 \mathrm{ml}$ of $0.01 \mathrm{~N}$ hydrochloric acid and $1 \mathrm{ml}$ of $\mathrm{Na}_{2}$ EDTA solution ( 94.6 $\mu g)$ was added. The solutions were heated to form the EDTAcomplexes, then mixed with $3 \mathrm{ml}$ of a solution containing 28.6 $\mu \mathrm{g}$ of cadmium which is equivalent to the amount of EDTA added. The polarographic reduction peak of the uncomplexed cadmium was then measured in the subtractive mode of operation against a procedure blank which had been carried through the entire procedure. The uncomplexed cadmium reduction peak measured results from its displacement from the EDTA-complex by metals present in the sample which form stronger EDTAcomplexes at this pH. Cadmium originally present in the samples would also contribute to the measured reduction peak. The results are given in Table 14.

Table 14. Total EDTA-complexed metals in perchloric acid.

Sample

Brand A

\section{Concentration, $\mathrm{ng} / \mathrm{g}$}

40

43

Brand $B$, Lot $X$

170

Brand $B$, Lot $Y$

88

Ordinary Commercial ACS spec. reagent
250

b. Nitric Acid and Hydrochloric Acid. Samples of commercially purified nitric acid and hydrochloric acid were received for evaluation for suitability for use in the determination of trace amounts of iron. Samples of $100 \mathrm{ml}$ were evaporated to dryness in quartz, the residues dissolved in $0.05 \mathrm{ml}$ of dilute hydrochloric acid, and diluted with an oxalic acid-ammonium oxalate supporting electrolyte. Iron was measured polarographically and found to be $53 \mathrm{ppb}$ ( $\mathrm{ng} / \mathrm{g}$ ) for the nitric acid and $19 \mathrm{ppb}$ for the hydrochloric acid. 
Bismuth is an interference in this procedure and hence would be included in the result above. Subsequent analysis by another technique of a different lot of purified nitric acid from the same supplier showed bismuth to be about $25 \mathrm{ppb}$. Bismuth interference can be eliminated by the use of the supporting electrolyte, ammonium acetate-EDTA, in which iron is reduced at about $-0.2 \mathrm{~V}$ and bismuth at about $-0.7 \mathrm{~V}$. A repetition of the analysis in this latter electrolyte gave an iron value of $50 \mathrm{ppb}$ for the nitric acid and $14 \mathrm{ppb}$ for the hydrochloric acid, which are considered to be in good agreement with the previous results.

c. Ammonium Hydroxide. A solution of ammonium hydroxide prepared by $G$. Sleater of the Separation and Purification section was submitted for determination of iron, copper, lead, and cadmium: This reagent had been prepared by passing tank ammonia gas through distilled water for 5 l/2 hrs. The sample was analyzed as follows: Triplicate 100-ml portions were evaporated to dryness. The residues were dissolved in $0.1 \mathrm{~N} \mathrm{HCl}, 5 \mathrm{ml}$ of an oxalic acid-ammonium oxalate solution was added, and iron, copper, cadmium and lead were determined polarographically. The results are shown in Table 15.

Table 15. Analysis of ammonium hydroxide.

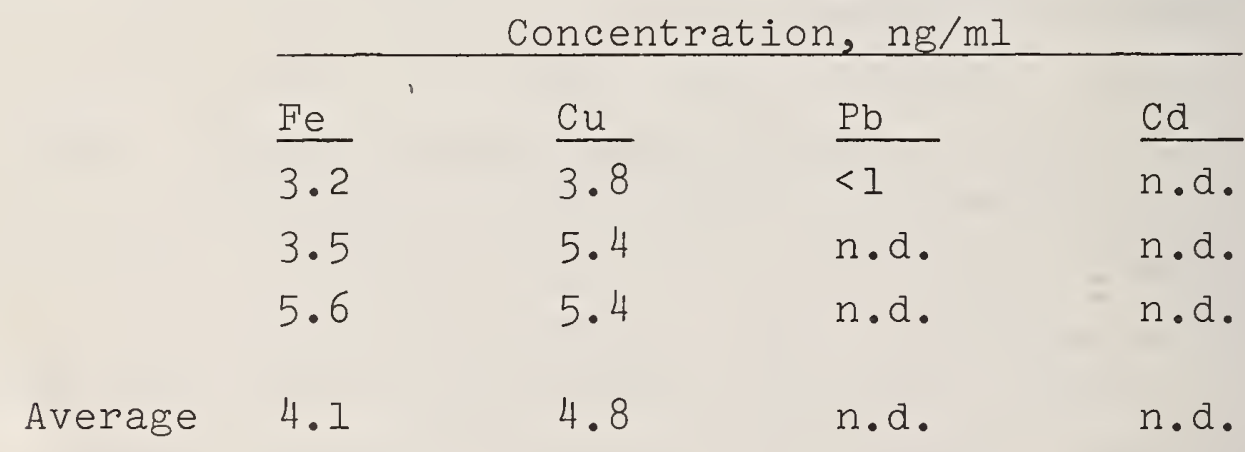

Under the conditions of this experiment the detection limit was less than $0.5 \mathrm{ng} / \mathrm{ml}$. 
2. Environmental Analysis

a. Laboratory Air. The contamination from the ambient air of samples undergoing chemical processing is a serious problem in trace analysis. The potential danger of introduction of foreign material is obvious but the quantitative aspects are difficult if not impossible to fully evaluate. Experience over many years has shown fluctuations in blanks that could only be ascribed to accidental contamination from the surrounding air.

A number of analyses have been made during the past year to determine the quality of the air in a typical laboratory, in the clean-air hoods described earlier in this chapter ( $p$. 44), and in the class 100 clean room of this Division.

Samples were collected on acid-washed, $47 \mathrm{~mm}, 0.8 \mathrm{\mu m}$ pore size membrane filters, using the house vacuum line with a 10-1/min limiting orifice inserted in the outlet connector of the filter holder. From about 30 to $100 \mathrm{~m}^{3}$ of air were sampled. The results are shown in Table 16.

Table 16. Particulates in laboratory air.

Concentration, $\mu \mathrm{g} / \mathrm{m}^{3}$

$\underline{\text { Room }}$

B309 high

Cu

Fe

0.03

$\underline{\mathrm{Pb}}$

0.6

$\underline{\mathrm{Cd}}$

low

0.1

0.003

0.1

0.002

B325 high

0.3

0.04

0.7

0.001

low

0.1

0.01

0.3

0.007

B323

0.2

0.01

0.2

0.001

0.001

The new class 100 laminar flow clean hoods were also monitored when they were first installed in room B323. Air samples from 170 to $350 \mathrm{~m}^{3}$ were collected and the polarographic results are shown in Table 17 together with those from similar studies of the clean room A306. This and the above work was 
done in collaboration with R. A. Paulson also of the Microchemical Analysis Section.

Table 17. Particulates in clean room air.

Location

B323 clean hood \#4 A306 clean room

\begin{tabular}{llll}
\multicolumn{4}{c}{ Concentration, $\mu \mathrm{g} / \mathrm{m}^{3}$} \\
\hline Fe & $\frac{\mathrm{Cu}}{0.007}$ & $\frac{\mathrm{Pb}}{0.0003}$ & $\frac{\mathrm{Cd}}{0.0002}$ \\
0.0009 & 0.007 & 0.0002 & n.d.
\end{tabular}

A comparison of the results given in Tables 16 and 17 show the marked improvement provided by clean facilities. To determine the size of blank resulting from atmospheric exposure of a typical sample container during processing, beakers with internal surface areas of about $0.012 \mathrm{~m}^{2}$ were allowed to sit in various laboratory areas for 6 days. Iron, copper, lead, and cadmium were then determined polarographically and the results are shown in Table 18.

Table 18. Fallout in laboratory and clean room air.

\begin{tabular}{|c|c|c|c|c|}
\hline & \multicolumn{2}{|c|}{ Fallout, $\mu g /$} & days $/ 0$ & $m^{2}$ \\
\hline Location & $\mathrm{Fe}$ & $\mathrm{Cu}$ & $\mathrm{Pb}$ & $\underline{\mathrm{Cd}}$ \\
\hline clean hood \#0 & 0.010 & 0.010 & 0.015 & ---- \\
\hline clean hood \#4 & 0.027 & ----- & ----- & --- \\
\hline $\begin{array}{l}\text { conventional lab } \\
\text { hood }\end{array}$ & 0.042 & ----- & 0.008 & 0.006 \\
\hline
\end{tabular}

Iron is seen in all cases to be high enough to limit the polarographic sensitivity obtainable. Copper and lead in hood 0 and lead and cadmium in the regular lab hood are also higher than the polarographic detection limit and, accordingly, would make significant contributions to the blank.

Experiments will be carried out to determine the titanium and aluminum blanks from the regular laboratory hood and the laminar flow hoods in the near future. A titanium pigment- 
epoxy paint was used in the laminar flow hoods and conceivably could contribute to a titanium blank. Also, large areas of aluminum are present as separators in the filter, possibly adding to the already high aluminum blank. It is especially important to have clean hoods which will lower the iron, aluminum and titanium blanks rather than contribute to them. Studies made in the Spectrochemical Analysis Section indicate that these elements (as well as others) may be present in amounts greater than 10 percent in ordinary laboratory dust fallout. In some recent determinations in this laboratory of traces of iron and aluminum in orchard leaves, the total blanks ranged from 1 to 3 micrograms. Applying the rule that the total signal from the sample should be twice that of the blank, this would limit the amount determined to several micrograms, whereas sufficient sensitivity is available to determine several nanograms.

b. Industrial Air. To illustrate polarographic contributions in the solution of a typical industrial hygiene problem, a recent example will be described. The NBS Glass Section needed to prepare several special batches of leaded glass in a general purpose laboratory; however, safety regulations require that the lead content of the air in such a case should not exceed $200 \mu \mathrm{g} / \mathrm{m}^{3}$. Samples collected under various conditions were submitted along with a blank filter. The procedure used was as follows: The samples were ashed in a low temperature asher at $200^{\circ} \mathrm{C}$ and the residues dissolved by heating with nitric acid. Hydrochloric acid was added and the solutions were again evaporated to dryness. The residues were dissolved in $10 \mathrm{ml}$ of $0.1 \mathrm{~N}$ hydrochloric acid and measured subtractively on a cathode ray polarograph at about $-0.5 \mathrm{~V}$ vs. a mercury pool anode using the blank filter which had been carried through the procedure as a background blank. Results of about 0.9 to 8 micrograms showed the lead particulate content to be well within safety regulations. 


\section{Lead in Fish Protein Concentrate.}

This laboratory has cooperated with the U.S. Bureau of Commercial Fisheries for several years in the determination of impurities in fish protein concentrate (FPC) intended for human consumption. During the past year, a number of analyses were made to compare the lead content of concentrates prepared from several kinds of fish.

Samples of either fish or protein concentrate were dry-ashed: the residues were dissolved in dilute hydrochloric acid, and iron was reduced with hydrazine hydrochloride. The lead was then extracted with sodium diethyldithiocarbamate and chloroform. The organic material was destroyed, after which the lead reduction peak was measured polarographically in $0.1 \mathrm{~N} \mathrm{HCl}$. The values obtained in previous years as well as most of the present values ranged from several tenths to about $1 \mu \mathrm{g} / \mathrm{g}$ of FPC, but those for FPC prepared from anchovies were about $5.5 \mathrm{\mu g} / \mathrm{g}$ of FPC. In order to verify that the lead was coming from the anchovy and not introduced during the processing, samples of raw fish were analyzed. The anchovy again showed a similar high level of lead. We have been informed that these analyses provide the basis of a decision not to use anchovies from this location for the preparation of FPC. The studies also showed a variation in lead content with the geographic area from which the fish were taken.

4. Copper and Zinc in Blood Plasma Processing Intermediates.

Determinations of copper and zinc in saline solutions and sodium lactate solutions used in processing blood were made for the American National Red Cross. Either one or both of the solutions provided were suspected of being contaminated with copper or zinc. These elements could be determined directly in the sample using the solution itself as a supporting electrolyte provided a suitable calibration curve could be established. For the curve to be valid, known amounts of copper and zinc should be added to solutions simulating the composition of the 
samples. However, no sodium chloride or sodium lactate of known purity was available, so the method of standard additions was used, which consisted of adding aliquots of copper and zinc solutions prepared from high purity melting point copper and melting point zinc to 5-ml aliquots of the samples. The results of the analysis are shown in Table 19.

Table 19. Copper and zinc in sodium lactate and sodium chloride solutions.

\begin{tabular}{|c|c|c|}
\hline \multirow[b]{2}{*}{ Samples } & \multicolumn{2}{|c|}{ Concentration, $\mu \mathrm{g} / \mathrm{ml}$} \\
\hline & $\underline{\mathrm{Cu}}$ & $\underline{\mathrm{Zn}}$ \\
\hline \multirow[t]{2}{*}{ 1\% Sodium chloride } & 0.23 & 0.30 \\
\hline & .15 & .32 \\
\hline Average & .19 & .31 \\
\hline \multirow[t]{2}{*}{ 1\% Sodium lactate } & $3 \cdot 3$ & 1.9 \\
\hline & 3.5 & 1.5 \\
\hline Average & 3.4 & 1.7 \\
\hline
\end{tabular}

It was concluded that the sodium lactate solution was not of sufficient purity for use in blood processing.

5. Lead, Bismuth and Tellurium in Special Steels.

A series of 5 special steels and irons have been developed for issue as general purpose standard reference materials for the iron and steel industry. These materials were prepared by special melting, casting and fabrication techniques and consist of a graded series of some 36 elements covering the useful concentration ranges required by the iron and steel industry. Polarographic methods have been developed and used for the determination of lead, bismuth, and tellurium in these special steels. Because these elements are present only in trace amounts, conventional chemical methods are not very satisfactory. The 
procedures are as follows: For lead and bismuth, 5-g samples of SRM 361 and SRM 1261 and $0.5-g$ samples of SRM 1178 were dissolved in hydrochloric and nitric acids, evaporated to dryness, dissolved in $\mathrm{HCl}$ and diluted to $50 \mathrm{ml}$ with concentrated HCl. The silica was allowed to settle after which 10-ml aliquots were taken and the bulk of the iron was extracted with isobutyl acetate. The aqueous layer was evaporated to dryness and the residue dissolved in 10 drops of hydrochloric acid, then in $15 \mathrm{ml}$ of water. Lead and bismuth were extracted with sodium diethyldithiocarbamate and chloroform from an ammoniacal solution containing cyanide and tartrate to complex interferences. After elimination of organic material, lead and bismuth were determined polarographically. SRM 1178 was used as an analytical control. Values of 0.013 percent bismuth and 0.004 percent lead were obtained for SRM 1178 which is certified to contain 0.013 percent bismuth and $0.00_{4}$ percent lead. The results for the special steels are shown in Table 20. Although comercially purified acids were used and all other reagents were purified by pre-extraction of lead, a reagent blank of approximately $1 \mu g$ of lead was obtained.

Table 20. Lead and bismuth in special steels.

Sample

361-composite

Average

1261-composite B $(2 \mathrm{~A}+2 \mathrm{C})$

Average
Metal content

\begin{tabular}{cc}
\hline Bi,ppm & Pb, ppm \\
4.4 & .22 \\
5.7 & .17 \\
5.0 & .20
\end{tabular}

$4 \cdot 1$

.20

$4 \cdot 3$

.22

$4 \cdot 2$

.21 
The major part of this blank has been proven to come from the reagents. Earlier work has shown none to come from the containers and the fallout studies discussed in Section 2.a show an expected contribution of only $8 \mathrm{ng}$, or less than 1 percent of the total lead blank, attributable to the atmosphere.

For the determination of tellurium, 10-ml aliquots of the solutions described above were taken. Tellurium was separated from the iron by precipitation with sulfur dioxide using a selenium carrier [17]. After filtration, destruction of organic material and volatilization of selenium, tellurium was determined polarographically. The results are shown in Table 21 .

Table 21. Tellurium in special steels.

$\underline{\text { Sample }}$

361-composite

126I-composite B $(2 \mathrm{~A}+2 \mathrm{C})$
Tellurium, percent

0.00041

0.00039

0.00051

0.00046

Essentially, the same procedure was also used to ascertain conformance with specification of a forthcoming addition to this series of special steels. Average values of 0.012 percent lead, $s=0.0001,0.020$ percent bismuth, $s=0.001$; and 0.0043 percent tellurium, $s=0.0003$, were obtained.

6. Titanium in Special Steels and Ductile Irons.

Procedures were developed and used to determine titanium in ductile iron as well as in the special steels provided above. Titanium in ductile iron SRM 1140 was determined on triplicate 0.5-g samples. Titanium was separated by precipitation first with cupferron, and then with ammonium hydroxide. After destruction of organic material and evaporation to dryness, 
the titanium residue was dissolved in sulfuric acid $(1+1)$ and diluted with an oxalic acid-ammonium oxalate supporting electrolyte. Titanium was then measured polarographically and the results are given in Table 22, along with results obtained by a cooperating analyst.

Table 22. Titanium in ductile iron SRM 1140.

\section{Titanium, percent}

$\begin{array}{ll}\text { Polarography } & \text { Cooperator A } \\ 0.093 & \\ 0.095 & \\ 0.098 & \end{array}$

Average $\quad 0.095$

0.094

Titanium in special steels, SRM 365 and SRM 1265, was determined using $1-g$ samples. In this case the bulk of iron was extracted with isobutyl acetate from a hydrochloric acid solution. The acid extract was evaporated to dryness and the titanium determination finished as already described for SRM 1140. The results are shown in Table 23.

Table 23. Titanium in SRM 365 and SRM 1265.

Sample

SRM 365
Titanium, percent

0.00072

0.00064

0.00052

0.00061

Average

0.00061

SRM 1265

$\begin{array}{cc} & 0.00052 \\ & 0.00052 \\ \text { Average } & 0.00052\end{array}$




\section{Nickel, Iron, and Titanium in Glass.}

The concept of glass-base trace element standard reference materials as well as preliminary work and method development were described in the 1969 report of this Section [12]. Work has continued in this laboratory on the determination of trace elements in glass standard reference materials.

The method developed here for nickel in nickel-doped soda lime glass has been applied to its determination in the 500-ppm glass and has been modified for the l-ppm glass with excellent results. The modified method will also be applicable to the remaining standards. Excellent agreement with results by another technique (spectrophotometry) was also obtained for the 500-ppm glass. No other technique has so far determined nickel in the l-ppm standard. The method developed is given below.

Wafers weighing approximately $1 \mathrm{~g}$ were cleaned first with alcohol and then with dilute nitric acid. The samples were dried, weighed, dissolved in hydrofluoric acid, and fumed with perchloric acid. After dilution to $50 \mathrm{ml}$, addition of sodium citrate, hydroxylamine hydrochloride, and ammonium hydroxide, nickel was extracted with dimethylglyoxime and chloroform. The chloroform was evaporated, organic material destroyed, and the nickel reduction peak was measured at about -1.0 $\mathrm{V}$ in a pyridine-pyridinium sulfate supporting electrolyte. The results are shown in Table 24.

Results later obtained by $E$. R. Deardorff of the Analytical Coordination Chemistry Section using spectrophotometry agreed exactly with the $450 \mathrm{ppm}$ value obtained in this laboratory.

By a minor modification involving limitation of total volume, the method described above can be applied to the l-ppm glass. Although the previous literature reports that at least $5 \mathrm{\mu g}$ must be present to insure complete extraction, it was noted that most of these methods used relatively large volumes during the extraction. By keeping the extraction 


\begin{tabular}{|c|c|c|c|c|c|c|c|}
\hline Set No. & \multirow{2}{*}{$\frac{1}{450}$} & \multirow[t]{2}{*}{2} & \multirow[t]{2}{*}{3} & \multirow[t]{2}{*}{4} & \multirow[t]{2}{*}{5} & \multirow[t]{2}{*}{6} & \multirow{2}{*}{$\begin{array}{c}\begin{array}{c}\text { Sample } \\
\text { avg. ppm }\end{array} \\
455\end{array}$} \\
\hline Sample No. $2 \mathrm{~A}$ & & & & & & & \\
\hline & 459 & & & & & & \\
\hline & 455 & & & & & & \\
\hline \multirow[t]{2}{*}{$13 \mathrm{~A}$} & & 458 & & & 449 & 441 & 450 \\
\hline & & & & & & 450 & \\
\hline $18 \mathrm{~A}$ & 442 & & 442 & & & 451 & 445 \\
\hline \multirow[t]{2}{*}{$48 \mathrm{~A}$} & & 463 & & 441 & 447 & 447 & 451 \\
\hline & & & & & & 459 & \\
\hline \multirow[t]{2}{*}{$56 \mathrm{~A}$} & & & 441 & 439 & & & 443 \\
\hline & & & 448 & & & & 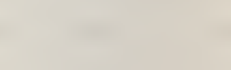 \\
\hline \multirow[t]{3}{*}{$66 \mathrm{~A}$} & & 459 & & & 446 & 455 & 455 \\
\hline & & 464 & & & 456 & 447 & \\
\hline & & 461 & & & & & \\
\hline \multirow[t]{2}{*}{$78 \mathrm{~A}$} & & & 444 & 451 & & & 446 \\
\hline & & & & 444 & & & \\
\hline \multirow[t]{2}{*}{$106 \mathrm{~A}$} & & & 444 & 445 & & & 445 \\
\hline & & & & 446 & & & \\
\hline
\end{tabular}

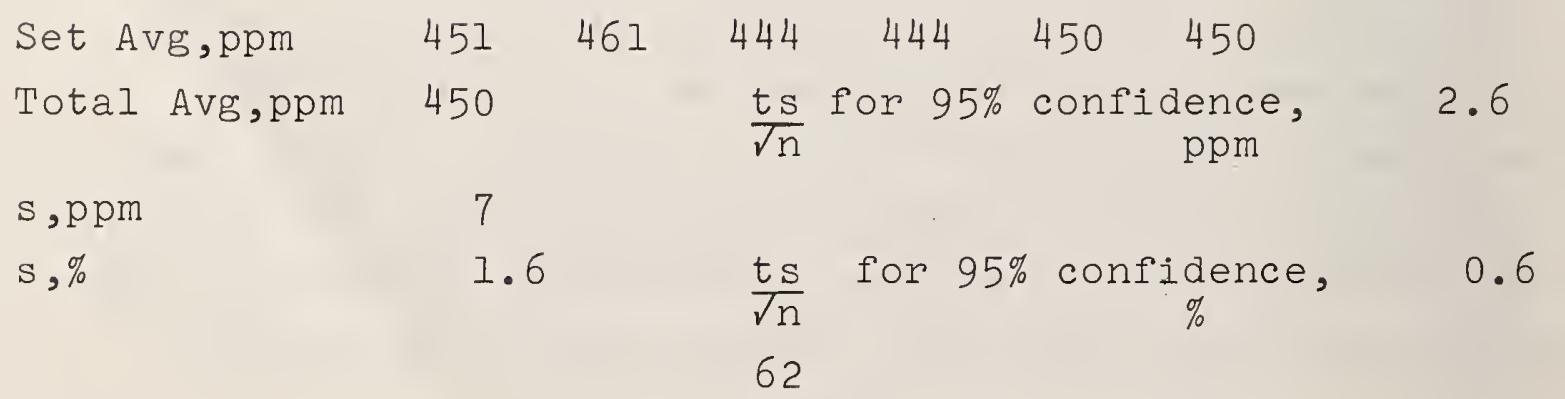


volumes as small as possible, it seemed probable that a microgram or less could be extracted. Accordingly, the volume of the aqueous layer was kept at 7-8 $\mathrm{ml}$ and it was found that as little as $0.5 \mu \mathrm{g}$ of nickel could be quantitatively extracted.

Nickel in 1-g samples of the 1-ppm glasses was then determined as just described and the results are given in Table 25 .

Table 25. Nickel in 1-ppm Glass

\begin{tabular}{|c|c|c|}
\hline Portion & Ni, ppm & Sample Average \\
\hline \multirow[t]{3}{*}{2} & 0.84 & \\
\hline & 0.85 & \\
\hline & 0.84 & 0.84 \\
\hline \multirow[t]{2}{*}{36} & 1.05 & \\
\hline & 0.98 & 1.02 \\
\hline \multirow[t]{2}{*}{40} & 0.91 & \\
\hline & 0.85 & 0.88 \\
\hline \multirow[t]{2}{*}{69} & 1.03 & \\
\hline & 1.06 & 1.04 \\
\hline \multirow[t]{2}{*}{76} & 0.97 & \\
\hline & 0.93 & 0.95 \\
\hline \multirow[t]{2}{*}{114} & 0.97 & \\
\hline & 1.01 & 0.99 \\
\hline \multirow[t]{3}{*}{ Sample } & Average, ppm & 0.95 \\
\hline & $\mathrm{s}, \mathrm{ppm}$ & 0.08 \\
\hline & s, \% & 8.4 \\
\hline
\end{tabular}

The method developed previously [12] for the determination of iron and titanium in soda-lime glass appeared to be applicable to the trace glass standards, with some modifications. Two supporting electrolytes had been investigated -EDTA-sodium acetate and sulfuric acid-sodium pyrophosphate. 
Because problems had recently been experienced with keeping the titanium in solution using the pyrophosphate supporting electrolyte, it was eliminated as a possibility. Investigation of an oxalic acid supporting electrolyte showed good applicability in the 1-10 $\mathrm{kg}$ range, so it was used for the 0.02-ppm and the base glasses.

After dissolution of the samples and volatilization of $\mathrm{HF}$ as already described for nickel, iron and titanium were extracted with cupferron and chloroform. The chloroform was removed by evaporation, the organic material was destroyed, and the iron and titanium reduction peaks were measured subtractively using, in the second cell, a blank carried through the procedure. Iron blanks were somewhat high as discussed earlier in Section 2a; values ranging from 1 to $3 \mu g$ were obtained. The results on the base and $0.02-p p m$ glasses are given in Tables 26 and 27.

Table 26. Iron and titanium in base glass.

Position

8

31

41

61

87

107

117

131
Fe, $\mathrm{ppm}$

$11.98,12.99$

12.29

13.05

$12.25,13.07,12.78$

13.68

12.46

12.35

12.52

12.67

0.49

Standard Deviation

$\mathrm{ppm}$

ts for $95 \%$

$\sqrt{n}$ confidence, ppm 0.34
Ti, ppm

$2.70,2.58$

2.78

2.86

$3.07,2.43,2.60$

2.80

2.88

3.02

2.75

2.77

0.19

0.13

At first, there was speculation over the possible reversal of samples, since the 0.02-ppm glass showed somewhat lower 
Table 27. Iron and titanium in 0.02-ppm glass.

Position

2

33

43

51

61

76

130
Fe, ppm

$11.16,11.39,10.78$

9.96

10.54

10.47

10.25

10.16

$11.49,12.35,11.92$

10.95

0.77

$\mathrm{ppm}$

ts for $95 \%$

$\sqrt{n}$ confidence, ppm
Ti, ppm

$2.33,2.39,2.63$

2.14

2.84

2.73

2.67

2.59

$2.17,2.52,2.38$

2.49

0.23

0.16

amounts of iron and titanium than the base glass. As results for other elements came in, however, the same apparent reversal persisted. It was then postulated that the base glass, which had been prepared first, had in effect, cleaned the walls of the melter of certain impurities and in so doing contaminated itself. No other values have been reported for iron and titanium as yet.

Iron and titanium were then determined in the 1-ppm glasses using the same procedure, except dilute hydrochloric acid was used instead of dilute nitric acid to clean the glass wafers. It was felt that hydrochloric would be a better solvent for adventitious iron or titanium on the wafer surface. Single or duplicate determinations were made at six different sample positions. Average values of $13.5 \mathrm{ppm}$ of iron with a standard deviation of $0.7 \mathrm{ppm}$ and $3.1 \mathrm{ppm}$ of titanium, with a standard deviation of $0.15 \mathrm{ppm}$ were obtained. No other values have been reported as yet.

For the 50-ppm glass, an additional separation was necessary, as $50 \mathrm{ppm}$ each of possible interferences such as 
copper, vanadium and molybdenum had been added. These elements would be also extracted with cupferron, but could be separated by an ammonium hydroxide separation. To insure complete precipitation of iron and titanium and also a valid blank, zirconium was added as a carrier. Since bismuth, which was also added to the glasses, might not be completely separated, it was necessary to use a supporting electrolyte other than oxalic acid. (The bismuth peak potential in the oxalic acid supporting electrolyte is too close to that of iron for good peak resolution.) In the EDTA-sodium acetate supporting electrolyte discussed earlier, the bismuth peak potential is displaced to about $-0.7 \mathrm{~V}$ which is sufficiently far from both iron and titanium to provide good resolution. The 50-ppm wafers were analyzed using this, modified procedure and the results are shown in Table 28 .

Table 28. Iron and Titanium in 50-ppm Glass

Sample Designation

$2 \mathrm{~A}$

$37 \mathrm{~A}$

$44 \mathrm{~A}$

$68 \mathrm{~A}$

75

114

114

A verage

$\mathrm{s}, \mathrm{ppm}$

$\mathrm{s}, \%$ $\frac{\text { Fe, } p p m}{42.22^{a}}$

52.43

51.43

50.55

50.53

51.14

51.69

$51 \cdot 30$

0.75

1.5 $\frac{\text { Ti, ppm }}{40 \cdot 32^{\mathrm{a}}}$

49.91

50.65

49.78

51.03

50.36

48.16

49.98

0.31

.62

$a_{\text {Not included in average. }}$

The extremely low value obtained for $2 \mathrm{~A}$ is thought to be real and indicates a deviation in composition of this rod. Accordingly, it will not be used as a SRM. An average value of $51.7 \mathrm{ppm}$ of iron was obtained by $\mathrm{R}$. W. Burke, of the 
Analytical Coordination Chemistry Section by a spectrophotometric procedure, showing excellent agreement between the two techniques. No other values have been reported for titanium.

Iron and titanium were determined in the 500-ppm glass by essentially the same procedure as for the 50-ppm glass; however, some difficulty was experienced in keeping the titanium in solution at various steps of the procedure. For example, after fuming with perchloric acid to remove the hydrofluoric acid, a large amount of insoluble residue was obtained and addition of hydrochloric acid was necessary along with prolonged heating to redissolve it. Concentrated sulfuric acid is a better solvent for hydrolyzed titania, but the excessive precipitate of calcium and lead sulfates precluded its use at this stage. Reversing the order of the separations and precipitating with ammonium hydroxide before extraction with cupferron was unsuccessful because the bulky precipitate resulting from the presence of almost every element of the ammonium hydroxide group, including the rare earths, made thorough washing of the precipitate very difficult. In taking pure solutions of iron and titanium with zirconium added as carrier through the entire procedure, recoveries of 99.6 percent of the iron, and 99.4 percent of the titanium were obtained.

The first results for 15 separate determinations gave an average for iron of $461 \mathrm{ppm}$ with a standard deviation of $20 \mathrm{ppm}$. In an attempt to determine if this deviation resulted from actual sample inhomogeneity or arose from the procedure, duplicate aliquots were taken. Some errors from loss or contamination could, of course, occur during solution and before the aliquots were taken; but the bulk of the chemical processing occurs after the aliquoting. The results are shown in Table 29.

While the agreement between aliquots is somewhat better than that between separate samples, it is not enough to show conclusively whether sample inhomogeneities exist. Again, the average spectrophotometric value of $461 \mathrm{ppm}$ obtained by 
Table 29. Iron in 500 ppm-glass.

\begin{tabular}{|c|c|c|c|}
\hline Sample No. & Iron, ppm & $\begin{array}{l}\text { Aliquot } \\
\text { Average }\end{array}$ & $\begin{array}{l}\text { Sample } \\
\text { Average }\end{array}$ \\
\hline 2 & $\begin{array}{l}482^{a} \\
482^{a}\end{array}$ & 482 & 482 \\
\hline 18 & $\begin{array}{l}453^{a} \\
460^{a}\end{array}$ & 456 & 456 \\
\hline 56 & $\begin{array}{l}469^{a} \\
469^{a}\end{array}$ & 469 & 469 \\
\hline \multirow[t]{2}{*}{13} & $458^{a}$ & 458 & \multirow[t]{2}{*}{451} \\
\hline & $\begin{array}{l}458 \mathrm{~b} \\
447 \mathrm{~b} \\
441 \mathrm{~b}\end{array}$ & 444 & \\
\hline \multirow[t]{2}{*}{48} & $\begin{array}{l}447^{\mathrm{a}} \\
466^{\mathrm{a}}\end{array}$ & 456 & \multirow[t]{2}{*}{457} \\
\hline & $464^{b} \mathrm{~b}$ & 458 & \\
\hline 66 & $\begin{array}{l}458 \\
445 a \\
452 a\end{array}$ & $\begin{array}{l}458 \\
448\end{array}$ & 453 \\
\hline \multirow[t]{2}{*}{78} & $462^{a}$ & 462 & \multirow[t]{2}{*}{460} \\
\hline & $454^{b}$ & 457 & \\
\hline 106 & $\begin{array}{l}454^{a} \\
458^{a}\end{array}$ & 456 & 456 \\
\hline $\begin{array}{l}\text { I sample av } \\
\text { rd deviatio } \\
\text { rd deviatio }\end{array}$ & $\begin{array}{l}\text { ge, } p p m \\
\text { ppm } \\
\%\end{array}$ & & $\begin{array}{r}460 \\
10 \\
2.2\end{array}$ \\
\hline
\end{tabular}

Overall sample average, ppm

Standard deviation, ppm

Standard deviation, \%

auplicate aliquots

${ }^{b}$ Duplicate aliquots of second solution

R. W. Burke is in excellent agreement with the polarographic results. Sample 2 was significantly and consistently higher in iron, so it will not be included in the final SRM. The values obtained at the same time for titanium are shown in Table 30 . 
Table 30. Titanium in 500-ppm glass.

\begin{tabular}{|c|c|c|c|}
\hline Sample No. & Titanium, ppm & $\begin{array}{l}\text { Aliquot } \\
\text { Average }\end{array}$ & $\begin{array}{l}\text { Sample } \\
\text { Average }\end{array}$ \\
\hline 2 & $\begin{array}{l}432^{a} \\
437^{a}\end{array}$ & 434 & 434 \\
\hline 18 & $\begin{array}{l}427^{\mathrm{a}} \\
427^{\mathrm{a}}\end{array}$ & 427 & 427 \\
\hline 56 & $\begin{array}{l}447^{\mathrm{a}} \\
442^{\mathrm{a}}\end{array}$ & 444 & 444 \\
\hline 13 & $\begin{array}{l}430^{\mathrm{a}} \\
437 \mathrm{a} \\
417^{\mathrm{b}} \\
450^{\mathrm{b}}\end{array}$ & $\begin{array}{l}434 \\
434\end{array}$ & 434 \\
\hline 48 & $\begin{array}{l}427^{\mathrm{a}} \\
456^{\mathrm{a}} \\
428 \mathrm{~b} \\
433^{\mathrm{b}}\end{array}$ & $\begin{array}{l}442 \\
430\end{array}$ & 436 \\
\hline 66 & 415 & 415 & 415 \\
\hline 78 & $\begin{array}{l}419^{a} \\
423 a \\
449 b \\
449^{b}\end{array}$ & $\begin{array}{l}421 \\
449\end{array}$ & 435 \\
\hline 106 & $\begin{array}{l}444^{a} \\
451^{a}\end{array}$ & 448 & 448 \\
\hline $\begin{array}{l}\text { rall sample } \\
\text { hdard deviat }\end{array}$ & $\begin{array}{l}\text { age, ppm } \\
\text { ppm }\end{array}$ & & $\begin{array}{r}434 \\
10\end{array}$ \\
\hline
\end{tabular}

Niobium, which was also added to the glasses could probably have been analyzed at the same time as it is also separated with the iron and titanium and its reduction peak follows that of titanium.

(E. J. Maienthal). 
A. Introduction

This laboratory has been concerned for a number of years with the analysis of small samples by classical microchemical techniques. Both organic and inorganic samples are analyzed with the work level about equally divided between the two categories. The laboratory has responsibility for maintenance of a clean room facility and provides microscopic and micromanipulation services to other projects of the Division. Some of the more interesting activities of the past year in the areas mentioned above are described in the following sections of this report.

B. Microchemical standards

This laboratory has the responsibility for the development and certification analysis of a series of standard reference materials specially suitable for calibration or analytical control of microchemical procedures. Two new materials have been added to this list of SRMs during the past year. One of these is highly purified urea for use in nitrogen determination while the second is bromobenzoic acid intended as a halogen standard. Preliminary work has been done on certification of chlorobenzoic acid and a search is in progress for procurement of a highly fluorinated organic compound.

\section{Urea, SRM 2141}

The NBS office of Standard Reference Materials presently issues Urea SRM 912 as a standard for clinical chemical analysis. A similar lot of highly purified urea has been procured, standardized, and has just been released for issue as a standard for the determination of nitrogen in organic materials containing large amounts of this element. Many of the present day automatic carbon-hydrogen-nitrogen analyzers compare samples through peak heights. Thus compounds of known high purity must be used to standardize the apparatus. More accurate results are obtained when the composition of the 
sample is close to that of a standard. The highest nitrogen in the present microchemical SRM series is only $11.4 \%$ nitrogen. Urea contains $46.65 \%$ nitrogen, hence, a more appropriate standard for high nitrogen determinations. Since knowledge of the nitrogen content is of considerable importance in many instances, highly reliable nitrogen values are often needed. The A.O.A.C. microchemical Kjeldahl method [18] was adapted for larger samples of higher nitrogen content. Fifty milligram samples were used and the ammonia was absorbed in $50 \mathrm{ml}$ of $0.05 \mathrm{~N} \mathrm{HCl}$. Preliminary experiments showed that the large amount of distillate required to carry over all of the ammonia resulted in excessive dilution which made the titration in the boric-acid methyl-purple indicator method less accurate. Accordingly, hydrochloric acid was used in the absorbing solution and the excess was determined by potentiometric titration with $0.05 \mathrm{~N} \mathrm{NaOH}$ to a $\mathrm{pH}$ of 5.5. Each distillation was run for ten minutes. To be certain that all of the ammonia had distilled over, the first absorber was replaced with a second one containing $5 \mathrm{ml}$ of boric acid. The ammonia thus absorbed was titrated directly with $0.05 \mathrm{~N} \mathrm{HCl}$ using methyl purple indicator. Under the conditions used, the second titration seldom exceeded $0.1 \mathrm{ml}$. The $0.05 \mathrm{~N} \mathrm{HCl}$ was standardized both by coulometric titration and by titration against [(Tris (hydroxymethyl) aminomethane)] SRM 723, a basimetric standard.

Table 31. Determination of nitrogen in urea SRM 2141.

Day

\begin{tabular}{|c|c|c|c|c|c|c|c|}
\hline Bottle & 1 & & 2 & & 3 & & 4 \\
\hline 1 & 46.54 & (1) & 46.58 & (3) & 46.66 & (4) & 46.66 \\
\hline 2 & 46.65 & (6) & 46.55 & (2) & 46.62 & (1) & 46.72 \\
\hline 3 & 46.63 & (5) & 46.64 & $(4)$ & 46.57 & (5) & 46.66 \\
\hline 4 & 46.65 & (2) & 46.62 & (6) & 46.65 & (3) & 46.61 \\
\hline 5 & 46.78 & (3) & 46.68 & (5) & 46.67 & (2) & 46.57 \\
\hline 6 & 46.71 & (4) & 46.62 & (1) & 46.54 & (6) & 46.62 \\
\hline
\end{tabular}


The urea was received from the supplier in six bottles. One sample from each bottle was analyzed on each of four days in a random order for a total of 24 determinations. The analytical results are given in Table 31. The numbers in parenthesis indicate the order of analysis on that day. The overall average found was $46.633 \% \mathrm{~N}$ with the standard deviation for a single measurement of $0.058 \%$. The standard error of the overall average is $0.0119 \%$ and the $95 \%$ confidence interval is 46.608 to 46.658. The theoretical value for nitrogen in urea is $46.659 \%$. There is no evidence of systematic differences due to order of analysis or between samples of the lot of material.

The nitrogen value of a sample of single crystal ammonium dihydrogen phosphate was determined at the same time. The average value of six determinations was $12.173 \% \mathrm{~N}$ with a standard deviation of the mean of 0.005 . The theoretical value is $12.178 \%$. Accordingly, it was concluded that there is no significant amount of bias in the analytical measurements.

An estimate of the homogeneity of the urea using small (0.6 mg) samples was made using a commercial carbon-hydrogennitrogen analyzer. The peak heights for each element in a series of six runs were measured and compared. The results are given in Table 32. The variation in peak height includes both any inhomogeneity of the sample and the variabjlity of operations and measurement. It is concluded that the urea

Table 32. Homogeneity studies of urea SRM 2141.

Avg peak height, $m m(\bar{x})$

$\mathrm{s}=$ standard deviation, $\mathrm{mm}$

Relative $\mathrm{s}=\frac{100 \mathrm{~s}}{\overline{\mathrm{x}}}, \%$

Theoretical composition, \%

Relative s at this composition

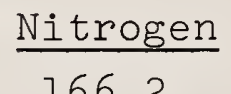

166.2

0.2

0.12

46.12

0.06
Carbon

171.3

0.8

.47

20.00

0.09

0.03 
is homogeneous in respect to those elements within the limits of precision of the analytical method. The moisture content of the urea was found to be $0.05 \%$ by the Karl Fisher titration method.

2. Intercomparison of Microchemical SRMS

A study was made of the present microchemical SRMs to ascertain whether there had been any change in these materials since they were first issued. Several of these standards are almost ten years old and one-Anisic Acid SRM 142-is almost 30 years old.

Benzoic Acid SRM 140b, Acetanilide SRM 14lb, Anisic Acid SRM 142, and Nicotinic Acid SRM 148 were analyzed at the same time in our laboratory and they showed a high inter-sample consistency in carbon and hydrogen and, in the two where it is present, nitrogen. The elemental compositions found were well within the expected limits of agreement with their theoretical proportions. Excellent agreement was also found in the phosphorus and sulfur content of triphenyl phosphate and cystine, respectively. In addition, benzoic, anisic and nicotinic acids were titrated with $0.1 \mathrm{~N}$ sodium hydroxide to obtain their acid equivalent values. The results of all of the determinations are summarized in Table 33.

Table 33. Purity of microchemical SRM's. Analytical measurement $\begin{array}{llll}\text { Micro } & \text { Micro } & \text { Micro } & \text { Micro } \\ \frac{\text { carbon }}{\%} \frac{\text { nitrogen }}{\%} \frac{\text { sulfur }}{\%} \frac{\text { Titrations }}{\%} & \frac{1}{\%}\end{array}$

Benzoic Acid SRM $140 \mathrm{~b}$ Acetanilide SRM $141 \mathrm{~b}$ Anisic Acid SRM 142

Cystine SRM 143b

Triphenylphosphate SRM 147 Nicotinic Acid SRM 148

100.1 100.10

$99.9 \quad 99.7$

99.9

99.96
$99 \cdot 7$ 100.4

$99.2 \quad 98.2$ 
While the results of Table 33 indicate that the SRMs were satisfactory to serve as microchemical standards, it was conceivable that a significant amount of decomposition not affecting the elemental analysis could have taken place. To eliminate any doubts of this nature, the materials were examined by several instrumental techniques including mass spectrometry, gas chromatography, paper and thin-layer chromatography, differential scanning calorimetry, and ultra-violet and infra-red spectrometry. Nuclear magnetic resonance spectra were also obtained. None of these techniques indicated any significant levels of impurities. Accordingly, the general conclusion was reached that these materials have not undergone any significant changes in composition and are entirely adequate for use as microchemical standards.

\section{Orchard Leaves, SRM 1571}

This sample of orchard leaves will be issued as a standard for the analysis of botanical matter. While the primary interest in it is perhaps the trace elements, it can also serve as a standard reference material for the determination of elements such as nitrogen and phosphorus in agricultural analysis. Accordingly, determinations of nitrogen and phosphorus were performed in this laboratory. In line with the recommendation of the A.O.A.C. methods, the samples were dried at $90^{\circ} \mathrm{C}$. for 24 hours under vacuum before analysis.

In the determination of nitrogen, the A.O.A.C. microchemical Kjeldahl method [18] was used with some modifications. Dried sample weights of approximately $0.25 \mathrm{~g}$ were digested with sulfuric acid, potassium sulfate and mercuric oxide and then distilled in the micro Kjeldahl distillation apparatus. As with the determination of nitrogen in urea (given above), the ammonia was absorbed in standard $0.05 \mathrm{~N}$ hydrochloric acid. The excess standard hydrochloric acid was titrated potentiometrically with $0.1 \mathrm{~N}$ sodium hydroxide to a $\mathrm{pH}$ of 5.6 . In this determination the initial hydrochloric acid was measured 
with a weight burette instead of by volumetric measurement with

a $50 \mathrm{ml}$ pipette. This gave better control over the amount of excess acid present as well as a more accurate measurement. The distillation was continued for 15 minutes since it was verified that all of the ammonia was distilled in this time. Although a second flask was added to check each determination, no ammonia was found in it.

The leaves were received in 20 containers. Since previous experience had indicated that a relative standard deviation of about 1 percent could be expected, it was decided to analyze one sample from each bottle and to do replicate analyses on any bottle that appeared to differ significantly in composition from the others. No such differences were found.

The results of the determination of nitrogen are tabulated in Table 34. The analytical sequence was downward in each column.

Table 34. Nitrogen in orchard leaves, SRM 1571.

$\begin{array}{rlrl}\begin{array}{c}\text { Bottle } \\ \text { number }\end{array} & \begin{array}{l}\text { Percent } \\ \text { nitrogen }\end{array} & \begin{array}{c}\text { Bottle } \\ \text { number }\end{array} & \begin{array}{l}\text { Percent } \\ \text { nitrogen }\end{array} \\ 18 & 2.76_{0} & 4 & 2.72_{9} \\ 19 & 2.78_{4} & 14 & 2.78_{1} \\ 15 & 2.74_{3} & 8 & 2.75_{9} \\ 5 & 2.74_{3} & 3 & 2.74_{5} \\ 7 & 2.72_{9} & 6 & 2.72_{3} \\ 17 & 2.76_{9} & 2 & 2.73_{4} \\ 11 & 2.77_{2} & 20 & 2.77_{4} \\ 9 & 2.76_{1} & 10 & 2.759 \\ 1 & 2.74_{3} & 16 & 2.752 \\ 13 & 2.77_{0} & 12 & 2.78_{0}\end{array}$

General average 2.755

Standard deviation .019

Standard deviation of the mean .004 
Phosphorus was determined in the same 20 bottles of orchard leaves using the A.O.A.C. microchemical method for phosphorus [19]. Dried weighed samples of $0.3 \mathrm{~g}$ were used. Each sample was digested with sulfuric acid and nitric acid until the organic material was decomposed. This digestion usually required about 5 hours. After dilution with an acid solution, the phosphorus was precipitated as ammonium phosphomolybdate, using ammonium molybdate reagent. After standing overnight, the precipitate was filtered through a weighed fritted glass micro filtering tube. It was found to be advantageous to use an ultrasonic bath to dislodge the last traces of precipitate from the walls of the precipitation flask. The precipitate was washed with ammonium nitrate solution, ethanol, and acetone and then dried for 30 minutes under vacuum at room temperature. The empirical factor of 0.01452 [20] was used in the calculations.

Table 35. Phosphorus in orchard leaves, SRM 1571.

\begin{tabular}{|c|c|c|c|}
\hline Sample & \% Phosphorus & $\underline{\text { Sample }}$ & \% Phosphorus \\
\hline 18 & 0.2088 & 13 & 0.2071 \\
\hline 15 & .2112 & 3 & .2106 \\
\hline 7 & .2065 & 8 & .2091 \\
\hline 17 & .2097 & 12 & .2062 \\
\hline 5 & .2081 & 10 & .2109 \\
\hline 4 & .2108 & 16 & .2067 \\
\hline 19 & .2079 & 1 & .2105 \\
\hline 9 & .2060 & 20 & .2083 \\
\hline 6 & .2082 & 11 & .2063 \\
\hline 14 & .2072 & 2 & $\frac{.2091}{0.2085}$ \\
\hline
\end{tabular}

Overall average $0.2085 \%$

Standard deviation $0.0017 \%$

Standard deviation of the mean $0.0004 \%$ 
The analytical results for all of the determinations of phosphorus are given in Table 35. The analytical sequence is downward and from left, then right.

The results obtained for both nitrogen and phosphorus show that the SRM is homogeneous with respect to each element, at the 0.25 to $0.30 \mathrm{~g}$ sample level, within the limit of the analytical determinations.

C. Ultramicrochemical Standards

Work on the evaluation of very small ion exchange beads as ultramicrostandards has continued (see reference [12] pg. 101). Beads ranging in diameter from 5 to $25 \mu \mathrm{m}$ have been prepared by the Separation and Purification Section of this Division and loaded with various cations. Since such beads furnish a concentrated source of a very small amount of an ion, they should furnish very small amounts of material for checking such procedures as detection limits, for example.

Beads loaded with ammonium ion were prepared and studied recently. Unlike many of the beads previously prepared, this surrenders its cation, ammonium ion, readily so is a convenient source of that ion. The use of these beads as standards for the microscopic spot test was studied, briefly. Lodge [21] has used such a technique for identification of air-borne particles but has not quantified it. The beads were dispersed on a microscope slide and the diameters of individual beads were measured using an eyepiece micrometer. Beads of appropriate size were selected and transferred to a white membrane filter. The color was developed by placing the filter on a drop of Nessler reagent and letting it soak through the paper for 30 seconds. The filter was then rinsed well with water and dried. The filter was examined under the microscope by both reflected and transmitted light and the diameters of the spots were measured with the eyepiece micrometer. Results of a typical experiment are given in Table 36. 
Table 36. Microscopic Nessler spot test.

\begin{tabular}{ccccc} 
Diameter of bead & Weight $\mathrm{NH}_{4}^{+}$ & & Diameter of stain, $\mu \mathrm{m}$ \\
\cline { 3 - 4 } & $\mathrm{ng}$ & & dark & light \\
95 & 51 & 300 & 1100 \\
52 & 8.2 & 150 & 750 \\
29 & 1.4 & & 80 \\
16 & 0.2 & & 40
\end{tabular}

The weight of contained ammonium ion was calculated by the formula $w=p \frac{\pi}{6} d$, where $d$ is the diameter of the bead and $\mathrm{p}$ is the partial density of the ammonium ion. These beads contained 8.0 percent ammonium ion by weight and the density was not measured but was assumed to be $1.4 \mathrm{~g} \mathrm{~cm}^{3}$, based on measurement of other beads. From this data, the value $p=$ 0.112 was calculated. When d is measured in micrometers, w is expressed in picograms.

A logarithmic plot of weight of ammonium ion with respect to diameter of stain (light) is linear although the precision was not as good as would have been desired. It is believed that this would be improved by further studies which are planned for the near future.

D. Research Material Analysis

1. Copper-Nickel and Copper-Palladium Alloys

The Alloy Physics Section of the Metallurgy Division prepared a series of copper-nickel and copper-palladium alloys for use in studies of corrosion mechanisms. Samples of approximately $0.5 \mathrm{~g}$ were available for analysis. Since spectrographic analysis confirmed a pure binary system, only one of the components, mainly copper, was determined and the second calculated by difference.

The copper-nickel alloys were analyzed by a conventional method, consisting of solution in nitric acid and electrogravimetric determination of the copper. Residual copper in the electrolyte was determined spectrophotometrically using the 
sodium diethyldithiocarbamate method. The alloys contained a small amount of black material insoluble in nitric acid. This residue was filtered on filter paper and ignited. The ignited residue ranged from less than 0.01 percent to 0.24 percent of the original sample. The insoluble matter appeared to be carbon and the analysis was corrected for its presence. The determination of palladium in the copper-palladium alloys provided a somewhat more complicated problem. Since both copper and palladium are electrodeposited under the same conditions, a pre-separation had to be made. A method developed by Gilchrist [22] at NBS a number of years ago was used to effect this separation. In this, palladium is complexed with sodium nitrite after which the copper may be hydrolytically precipitated from hot solution at pH 8 as the hydroxide. Since the precipitate is non-stoichiometric, it was filtered, redissolved in nitric acid and the copper was electrodeposited on tared electrodes.

In a few cases, the copper deposit was dark, indicating some codeposition of palladium as a result of coprecipitation. In such circumstances, the copper was redissolved in acid and the palladium was determined by the gravimetric dimethylglyoxime method. The copper deposit was then corrected for this palladium content which varied from 0.2 to $0.7 \mathrm{mg}$. A correction was also made for residual copper in the electrolyte, which ranged from 0.1 to $1.8 \mathrm{mg}$.

By the use of the procedure described above, $0.5 \mathrm{~g}$ samples were analyzed in duplicate for copper contents ranging from 27 to 96 percent. Duplicate analysis showed a maximum difference of 0.08 percent copper.

2. Tungsten-Rhenium Alloys

The Temperature Section of the Heat Division prepared a series of 19 tungsten-rhenium alloys with rhenium content ranging Irom 1 to 27 percent for special thermocouple studies. The initial step in the preparation consisted of mixing purified metal powders in proper proportion and subsequent formation into 
wires. Analyses accurate to 0.1 percent were needed for control of composition.

A method furnished by Cleveland Refractory Metals [23] was used with only minor modifications for the determination of the rhenium content. Weighed samples of the metal (50 mg for $27 \%, 2$ grams for $1 \%$ ) were dissolved in a $3 \%$ hydrogen peroxide solution. Both components dissolved under this condition, but the tungsten could be precipitated as the hydrous oxide by the addition of hydrochloric acid. This oxide was then redissolved and held in solution by the addition of tartaric acid and ammonia. The rhenium was then precipitated with tetraphenylarsonium chloride and 6 M ammonium hydroxide.

The tetraphenylarsonium-perrhenate was filtered on a tared fritted glass filter, washed with a saturated solution of tetraphenylarsonium-perrhenate solution, and dried. One sample was analyzed in triplicate for which the results, $26.74,26.65$, and 26.64 percent rhenium were obtained. Only single determinations were made on the remaining alloys.

3. Thermochemical Reaction Evaluation

Assistance has been given to the Thermodynamics Section in the analysis of residual sodium hydroxide from calorimetric measurements of the enthalpy of the reaction of ketene gas with aqueous sodium hydroxide in an adiabatic calorimeter, according to the equation [24].

$\mathrm{CH}_{2} \mathrm{CO}(\mathrm{g})+\mathrm{NaOH}\left(\mathrm{aq}, 50 \mathrm{H}_{2} \mathrm{O}\right) \rightarrow \mathrm{CH}_{3} \mathrm{COONa}$ (aq, $\mathrm{NaOH}$ solution) The best method for determination of the amount of reaction that had occurred appears to be a careful determination of the sodium hydroxide consumed. This was measured by analysis of the original solution and after the final reaction.

For the calorimetric experiments the initial concentrations of the sodium hydroxide solutions was either $2 \mathrm{~g} / \mathrm{kg}$ or $4 \mathrm{~g} / \mathrm{kg}$. Aliquots of original and final solutions were taken by weight, and amounted to $50 \mathrm{~g}$ in each case. These were titrated with $0.1 \mathrm{~N}$ hydrochloric acid in $\mathrm{CO}_{2}$-free air. Each 
was done in duplicate and differences reliable to $0.1 \%$ were obtained.

Because carbon dioxide was an impurity in the ketene and also in the original sodium hydroxide, it too had to be determined. This was determined gravimetrically after evolution with acid. The amount of carbon dioxide present in the residual sodium hydroxide solution averaged about $0.01 \mathrm{mg} / \mathrm{g}$.

The final solutions were also analyzed for acetone which could arise from polymerization of the ketene. An iodimetric technique was used for this purpose and as little as $0.1 \mathrm{mg}$ of acetone/50 g aliquot would have been detected which would have amounted to less than $0.05 \%$. No acetone was found.

4. Fluorinated Copolymer Analysis

Elemental microchemical analysis has been used to determine the composition of fluorinated copolymers for the NBS Polymer Division. These polymers were prepared by mixing $\mathrm{C}_{2} \mathrm{~F}_{4}$ with $\mathrm{C}_{3} \mathrm{~F}_{3} \mathrm{H}_{3}, \mathrm{C}_{5} \mathrm{~F}_{7} \mathrm{H}_{3}$, or $\mathrm{C}_{4} \mathrm{~F}_{6} \mathrm{H}_{2}$. When two monomers are combined to form a copolymer, the combining ratios are difficult to determine. However, if the monomers differ sufficiently in composition, determination of carbon or hydrogen in the copolymer can serve to establish the ratio. The carbon determination is the more precise procedure.

As an example of the technique, one set of these copolymers was prepared from $\mathrm{C}_{2} \mathrm{~F}_{4}$ and $\mathrm{C}_{4} \mathrm{~F}_{6} \mathrm{H}_{2}$ which have carbon values of 24.0 and 29.3 percent, respectively. Even though these values are not markedly different, precise analysis should provide useful information. To achieve acceptable precision, determinations were made in replicate using $5 \mathrm{mg}$ samples. A combustion tube packed with $\mathrm{MgO}$ was used to remove fluorine and the $\mathrm{CO}_{2}$ and water were absorbed and determined gravimetrically. The usual standard deviations for a single determination in microdeterminations of this kind are $0.10 \%$ for carbon and $0.05 \%$ for hydrogen, respectively. By running 5 to 10 replicates, the 95 percent confidence interval for the mean value approximates these limits. R. A. Paulson and W. P. Schmidt 
A. Nature of the Analytical Problem

Chemical analysis is one of the most important aspects of environmental surveillance and control. The atmosphere needs to be analyzed and often continuously monitored to ascertain compliance with or deviation from air quality standards. Such data are also essential for establishing criteria upon which air quality standards are based. Since measurements typically are made over long periods of time, must be comparable with those of other locations or stations, and must be of legal integrity to support compliance with standards, they must have a high degree of precision and accuracy.

The determination of atmospheric pollutants and contaminants involves a high degree of sophistication in trace analysis. Constituents of interest need to be measured down to the partsper-billion level in many cases. Consequently, analytical methods must be very sensitive as well as selective to minimize preseparation steps. Upon these requirements is often superimposed such specifications as portability, continuous monitoring capability, simplicity to enable use by semiskilled technicians, and low initial and maintenance cost. Accordingly, it is suprising that the state of the art is as well advanced as it presently is.

Chemical analysis for air pollutants involves several distinctive steps:

\author{
Interpretation \\ Sampling \\ Metrology \\ Physical Analysis \\ Chemical Analysis
}

* This chapter consists of an appraisal of the present state of the art of air pollutant analysis, identifies measurement problems concerned with such analyses, and suggests areas of research to provide improved measurement capability. 
Each of these steps is involved directly or indirectly to a greater or less extent in every determination. The details (sub-steps) of each step need to be designed for the particular application and must be of requisite reliability. When substeps are minimized or passed over, it must be assumed that they are not significant and the validity of such assumptions influences the validity of the entire measurement.

1. Interpretation

The use to be made of the data is a question that must be answered before any analysis is made. This is necessary to establish such matters as detection limits, accuracy, precision, and the fine structural requirements such as whether an elemental analysis or determination of a specific substance is required. Measurement methods such as the "dust bucket", "sulfur candle", or total oxidant may be meaningful or totally inadequate, depending upon the information they are intended to convey.

In adition to its critical input into method selection, a consideration of the end use of the data will establish sampling frequency and procedures, and provide for the design of experimental procedures to insure statistical adequacy.

The significance of analysis is illustrated by Figure 15. Analysis provides the numbers upon which decisions are made and is the major measurement science of environmental evaluation as well as other aspects of materials research. The diagram emphasizes the extreme importance of the related steps and their interdependence. The diagram also points out that research in environmental analysis can lead to better methods, techniques, and instrumentation, which conversely can lead to better analytical data. Furthermore, fundamental studies can provide input to better measurements which in turn are a requisite for better understanding of fundamental principles. 


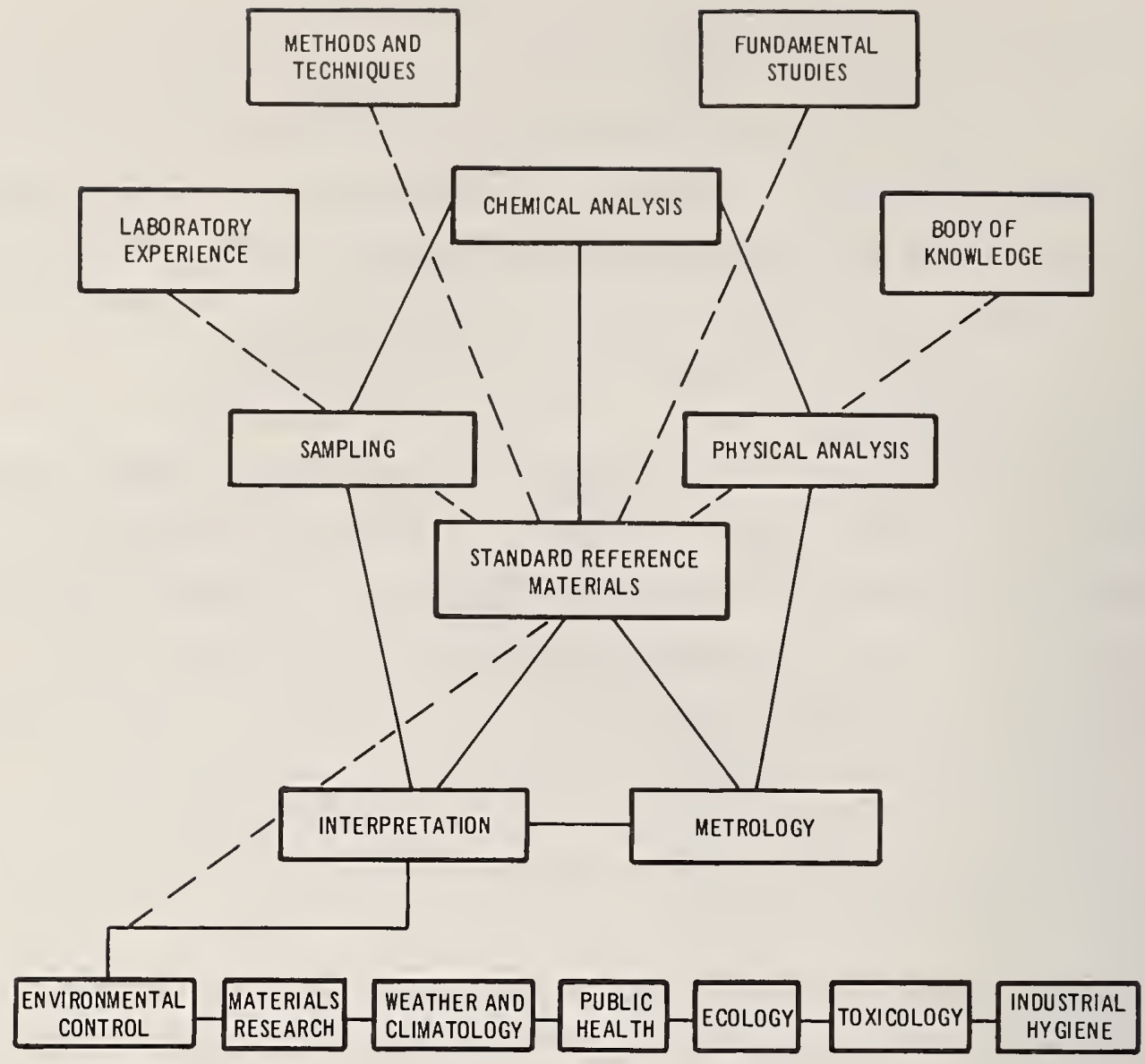

Figure 15. The analytical system for environmental measurements.

The lower part of the diagram indicates that while environmental control is important, it is only one of the products of environmental analysis. Many areas of industrial hygiene, toxicology, and public health depend on measurements closely similar to pollutant analysis. The presence of atmospheric contaminants, whether or not they are pollutants, has important implications in weather and climatology studies, on the 
stability of materials, and on ecological relations. There are many conflicting opinions in these areas that may have been generated by poor analytical data, and undoubtedly would be clarified by improved environmental analysis.

The diagram points out that environmental science seeks access to analytical measurements through the interpretation route, that is by posing questions that analysis will answer. It also suggests that analytical data properly interpreted can provide input into environmental science.

The diagram puts standard reference materials in the center because they provide a means to interrelate and verify the adequacy of each step in the analytical cycle. There are several laudable efforts in progress or proposed to provide better analytical methodology. The validity of their conclusions would be enhanced if the experimental design included the use of Standard Reference Materials required for method verification and/or calibration. Because of their intrinsic nature, most methods not only need, but require standards to verify their accuracy and insure their reliability.

\section{Sampling}

This step is an important part of every air pollutant analysis and may be most difficult to implement. Not only must one know what to sample, but how and when it should be done. The sampling technique must be such as to provide an adequate and representative sample. Furthermore, it must maintain the integrity of the sample up to the time of analytical measurement. When a delay is necessary between sampling and analysis, special precautions to maintain stability of a sample may be required. In this event, one technique that may be used is to react the sample with a reagent to produce a substance which is stable and can be measured subsequently. Traps, liquid impingers, and absorbers need to be designed for specific purposes. Gas samples may be collected in glass vessels, in tanks of selected materials or 
with special linings, or in plastic bags. Samples collected on filters need to be interpreted on the basis of pore size and filter composition, for example. Electrostatic and thermal precipitators may be used but the possibility of chemical alteration of some constituents of the sample is an impurtant consideration. The validity of the sampling technique needs to be verified in every situation.

3. Physical Analysis

Techniques included in this classification have to do with particulate analysis. The total atmospheric burden of particulates, the measurement of size and size-distribution, and the resulting modification of the atmosphere including light obscuration, fall-out on material and vegetation, and alteration of the esthetic aspects of the landscape are some of the areas related to such measurements.

The importance of relating the physical analysis with the chemical analysis is great, indeed, since an understanding of the adverse effects of many particulate pollutants depends on a knowledge of both size and composition. Thus, the physical analysis may dictate the sampling technique. The intimate relationship of physical analysis with other steps of the analytical system is at once obvious.

4. Metrology

Analytical measurements are typically related to a definite amount of total sample and expressed in concentration units. Often a preconcentration step is involved in which the final determination is related to a specified volume. Accordingly, measurement of rates of flow and cross-sectional areas may be required. Flow meters, limiting orifices, syringes, and gas burettes are some of the equipment for which calibration is required in such instances.

5. Chemical Analysis

This is the most obvious analytical step for which a variety of techniques are often available. Such methods may 
be entirely wet chemical, partially wet chemical, or entirely instrumental. For the purpose of this discussion, all of these are included in the above heading. A survey of the literature will show that many methods have been described and used for a given analysis with varying degrees of success. And yet, authoritative books and consultations with practicioners in the field indicate that better methods are urgently needed.

Part of the explanation of the above mentioned situation is related to the basic philosophy of air pollutant analysis. one school takes the "Least Common Denominator" approach. Methods should be as simple as possible, requiring a minimum of equipment, investment, and expertise. Another segment of technologists feel that devices similar to the familiar radiation monitor badges are needed to provide simple integrated exposure indicators. Such devices, if simple and inexpensive, would provide a wide base of monitoring at a "requisite level of precision".

On the other hand, others demand sophisticated equipment and are willing to pay a high price for apparatus and personnel to operate it. They feel that the high degree of reliability of the data would compensate for the problem of a more limited number of data acquisition centers.

The presently-used methodology appears to be a balance between these extreme points of view with simplicity playing a dominant influential role. Environmental scientists and engineers have been working on limited budgets and have had to be "practical". There is no doubt that the recent interest in air pollution is pushing air quality standards to more strigent limits and these will severely tax present analytical capability. The need to continuously monitor pollutants, to observe both short and long-term trends, and to predict incidents is well recognized. The need to make measurements in remote locations and to telemeter or otherwise transmit data to control centers will forge a new technology in pollution analysis. 
The general state-of-the-art of elemental analysis is much improved in recent years. Detection limits for many of the elements have been pushed down to parts-per-billion levels and lower, with absolute amounts of as little as 10,000 atoms having been reported as detectable in special situations. Such detection limits are, of course, seldom achieved and are perhaps unnecessary in pollution analytical applications. However, highly sensitive methods are very desirable because they provide the capability for short-time monitoring or minimize the amount of sample required. Also, there is every reason to believe that future air quality standards will be more stringent than those of the present time. Accordingly, the search for better and more sensitive methods is expected to be an active area of research in the foreseeable future. The state-of-the-art of proximate analysis, that is the identification and determination of specific radicals or compounds is less advanced. Detectors or sensors are often not sufficiently sensitive to the small differences between species present or to differentiate them from the matrix, so that separation techniques must be used. Indeed some of the most successful analytical techniques employ non-discriminatory but sensitive sensors, with reliance on the known parameters of the separator. Gas chromatography is a prime example of such techniques. By combining more specific identification techniques with highly efficient separation procedures, improved measurement techniques should result.

It would appear from analogy with other problems, that the basic scientific knowledge is already available to solve or ameliorate many of the analytical problems now facing environmental science. What then is responsible for the present relatively poor state-of-the-art of environmental analysis? The answer for this will be found in the following discussion. 
Referring back to Figure 15, this two-dimensional diagram may be expanded in the plane of the paper to provide an almost unlimited series of rings, each for a given analytical situation. In fact, one can conceive of an annulus with cross section equivalent to the ring of Figure 15. Similar rings could be sliced from the annulus to describe water analysis and steel analysis, for example. Each ring would include its own particular sub-units in each step, which may be common to other rings or may be distinguishably different. As a discipline (ring) stabilizes, new sub-steps are absorbed and old ones are rejected to provide a technology consistent with the requirements of the discipline.

The word requirement is the key to the situation. Until only recently, there has been little recognition of need and hence, there has been only limited support for research to develop analytical methods specifically suited for environmental analysis. Rather, techniques have been borrowed from other disciplines. Except in research establishments, measurements predominantly have been made by technicians or by engineers with little feel for analysis, and by the use of methods selected largely with economic considerations in mind. Moreover, there has been little incentive for research analysts to enter or contribute to the field, and instrument manufacturers have not seen a market which would stimulate the development of sophisticated instruments. The present concern about the environment has now made this a glamour field and with the availability of funding, new developments can be expected to emerge to greatly change the situation. There are still many problem areas and general and specific suggestions where contributions can be made are given in a succeeding section.

C. State of the Art of Standard Reference Materials

Standard reference materials for the evaluation of methods or the verification of their use in a given environmental 
analytical situation are almost nonexistent. Recognizing the general need for such materials, the Analytical Chemistry Division made proposals to the Office of Standard Reference Materials which have resulted in two sets of standard reference materials which are nearing completion:

Hydrocarbon in Air-4 standards:

1, 10, 100, and 1000 ppm respectively

Carbon Monoxide in Air-6 standards:

$10,50,100,300,500$, and 1000 ppm respectively.

These materials should provide a means for standardizing analytical data for these important measurements. However, because of the limited resources available at NBS for pollution standards work, and the fact that they were prepared in parallel with other efforts, a period of almost two years was involved in their certification. Obviously, a task force devoted to such standards would be required if a significant output would result. In addition to those mentioned above, NBS issues several other standard Reference Materials with air pollution relevance. These are listed in Table 37.

A NAPCA sponsored project at NBS has been concerned with a study of the feasibility of permeation tubes as analytical standards for $\mathrm{SO}_{2}$ determination. This work has established the degree of confidence that can be ascribed to such devices, and a project has now been established in the office of Standard Reference Materials to supply such tubes as Standard Reference Materials early in FY 1971.

A serious obstacle to the pollutant standards program is that the need for such materials is not generally recognized. As a result, meaningful intercomparison of analytical methods is impossible, due to lack of adequate test materials. Moreover, analytical results, in compliance questions, for example, may be subject to equivocation from two points: (1) validity of method, and (2) validity of application. 
Because of the nature of atmospheric analysis, the present state-of-the-art demands that both a standard method and a standard reference material be specified. If NBS is to provide such materials, a way must be found to expand its effort in this activity above the present level.

Table 37. Standard reference materials related to environmental analysis

SRM 1601-1603 Carbon dioxide in nitrogen containing 308, 346 , and $384 \mathrm{ppm} \mathrm{CO}$, respectively.

SRM 1604-1608 Containing 3, 10, 112, 212, and 978 ppm oxygen in nitrogen, respectively.

SRM 1609 Air with oxygen content certified at atmospheric level.

SRM 1610-1613 Containing 1, 10, 100, and 1000 ppm methane in air, respectively.

SRM 1616-1620 Containing 10, 50, 100, 300,500, and $1000 \mathrm{ppm}$ CO in air, respectively.

SRM 1621, 1622 Residual fuel oils, containing 1.05 and $2.14 \%$ sulfur, respectively.

$\mathrm{SRM} 1800$

Microstandard ion-exchange beads provide elemental standards at the microgram to nanogram level.

D. Analytical Methods for Specific Pollutants

The pollutants of the atmosphere may be classified as gases or particulates. Actually the latter might better be termed as aerosols since they consist of liquid droplets and solid particles, often colloidally dispersed in the air.

The gaseous materials ordinarily determined, either frequently or infrequently, are listed in Table 38. The kinds of measurements made for particulates are summarized in Table 39. Measurement of total particulates - mass per unit volume 
of air - is most frequently made, while chemical analysis is ordinarily reserved for selected samples.

For several years, the atmosphere has been under continuous surveillance by NAPCA's CAMP - Continuous Air Monitoring Program. This activity consists of stations that continuously determine the following: $\mathrm{SO}_{\mathrm{X}}, \mathrm{NO}_{2}$, $\mathrm{NO}$, CO, total oxidant, total hydrocarbons, total particulates. These stations, while limited in number, are located in places where municipal or state programs are active and with which they enjoy a degree of cooperation.

NAPCA's National Air Sampling Network collects samples taken at about 123 stations in a systematic manner and analyzes them at their headquarters, presently at Cincinnati. This system appears to work reasonably well, but there would seem to be problems concerned with stabilizing samples during the period between collection and analysis.

Municipal air quality centers, notably in the larger cities, have been in existence for several years. The State of California has been very active, and there is a good distribution of monitors throughout the state. In all of the above, the programs have good parallelism with CAMP.

The industrial situation is sporadic. Major companies monitor their facilities, especially their emission sources, such as stacks for example. Many power companies analyze their fuels or buy on tight specifications. The increased attention being given to such activities is encouraging.

Automobile manufacturers have major engineering programs for reduction of automotive emissions. As a consequence, they have laboratories to measure such emissions under various operating conditions and their findings have made important contributions to the literature. These companies have understandingly, been concerned about the production of smog and several have analytical laboratories dedicated to the study of this problem. 
Table 38. Analysis of gaseous pollutants.

Frequently Determined

$\begin{array}{ll}\text { Oxides of sulfur } & -\mathrm{SO}_{\mathrm{X}} \\ \text { Nitric oxide } & -\mathrm{NO} \\ \text { Nitrogen dioxide } & -\mathrm{NO}_{2} \\ \text { Carbon monoxide } & -\mathrm{CO}^{2}\end{array}$

Total hydrocarbons -

Methane

Total oxidants
Infrequently Determined

Hydrogen sulfide $\quad-\mathrm{H}_{2} \mathrm{~S}$

Mercaptans - R-SH

Hydrogen fluoride - HF

Peroxyacetyl Nitrate ( $P A N)$

Aldehydes

olefins

Ammonia

Specific organic

compounds

Table 39. Particulate characterization.

Total particulates

Size distribution

Specific fraction determination

Specific element determination

Specific substance determination

The complexity of atmospheric analysis can be expected to increase with the proposed publication by NAPCA of an impressive number of Air Quality Criteria Documents. The expected order of appearance of the documents is given in Table 40. Since the tolerance levels for many of these pollutants may be in the range of a few micrograms-per-cubic meter, their monitoring will involve highly sophisticated trace analysis which would seem to be beyond the level of capability reasonably to be expected from most of the present air quality stations. Accordingly, a system of laboratories designed for such determinations may be required. 
Table 40. Expected schedule of appearance of NAPCA air quality criteria documents.

\begin{tabular}{ll} 
Year & \multicolumn{1}{c}{ Pollutants } \\
1969 & $\begin{array}{l}\text { Sulfur oxides, particulates } \\
\text { Carbon monoxide, hydrocarbons, photochemical } \\
\text { oxidants }\end{array}$ \\
1971 & $\begin{array}{l}\text { Fluoride, lead, nitrogen oxides, polynuclear } \\
\text { organics, asbestos, beryllium }\end{array}$ \\
1972 & $\begin{array}{l}\text { Chlorine, hydrogen chloride, odors, arsenic, } \\
\text { cadmium }\end{array}$ \\
1973 & Copper, manganese, nickel, vanadium, zinc \\
1975 & Barium, boron, chromium, mercury, selenium \\
& Pesticides, radioactive materials
\end{tabular}

1. Gaseous Pollutants

The gaseous pollutants presently determined are listed in Table 38. The methods used will be found in several easily accessible reference works [25-28], to which the reader is referred for details. In general, these methods are essentially nonspecific and depend to a large extent on redox or acidimetric reactions which are colorimetrically measured. Instrumental methods, especially electrochemical, are becoming more popular but these, too, often suffer from interferences so that the algebraic sum of pollutants having similar properties is measured.

a. Sulfur Dioxide. The method of preference for umpire determinations would appear to be the pararosaniline (West-Gaeke) procedure which involves the formation of a redpurple pararosaniline methylsulfonic acid which is measured colorimetrically [29]. This procedure is suitable for $\mathrm{SO}_{2}$ concentrations in the range of 0.005 to $5 \mathrm{ppm}$ and is very selective but it is time consuming. However, the procedure may be automated. 
Other methods [30] such as conductimetry, titrimetry, turbidimetry, and iodimetry are in use but they lack specificity. A gas chromatographic method [1] has been recently proposed which may be the forerunner of a new lot of specific instrumental methods. A simple integrative method based on the rate of sulfation of lead peroxide coated on a cylindrical base, and dubbed the "sulfur candle" gives a measure of $\mathrm{SO}_{\mathrm{X}}$ exposure levels and is used in a number of countries.

b. Hydrogen Sulfide. This gas becomes an odor nuisance at the $0.1 \mathrm{ppm}$ level or less and is ordinarily present well below this concentration. The method presently used for its estimation consists in absorption in an alkaline suspension of cadmium hydroxide contained in an impinger, reaction with a mixture of p-aminodimethylaniline, ferric chloride, and chloride ion to yield methylene blue which is measured colorimetrically [31]. Gas chromatography is a promising technique for hydrogen sulfide determination [1].

\section{c. Nitrogen Oxides. Nitrogen dioxide is best} measured by a method developed by Saltzman [32], based on the Greiss-Ilosvay reaction. The $\mathrm{NO}_{2}$ is collected in the absorbing reagent consisting of sulfanilic acid, glacial acetic acid, and $N$-(l-napthyl)-ethylenediamine dihydrochloride with the production of a pink-colored dye complex by the diazotization reaction. The method is useful over the range from a few parts per billion to about $5 \mathrm{ppm}$. It is also applicable to the determination of nitric oxide, in which case it is oxidized to $\mathrm{NO}_{2}$ for subsequent determination.

The method is free from interferences from other nitrogen compounds, and a 5-fold excess of ozone or a 10-fold excess of $\mathrm{SO}_{2}$ causes negligible problems. The most serious problem appears to be the standardization. It has been empirically observed that 1 mole of $\mathrm{NO}_{2}$ produces the same amount of color as 0.72 mole of sodium nitrite which ordinarily serves as the standard; but this factor does not have universal acceptance. 
The availability of an SRM such as a certified $\mathrm{NO}_{2}$ permeation tube would appear to be a more satisfactory method of calibration.

The method has been semi-automated with commercially available instrumentation.

d. Ozone and Oxidants. Ozone and oxidants are determined because they serve as indices of eye irritants and plant-damaging pollution levels. The deleterious effects of such oxidants on materials such as rubber, for example, are also of importance. Oxidants are frequently determined by iodimetric procedures in which the absorbant potassium iodide solution is either buffered at the neutral point, or is made alkaline to eliminate $\mathrm{SO}_{2}$ interference. The latter modification provides stable containment for future determination in the laboratory.

Both methods are useful in the range of a few parts per hundred miliion to about $20 \mathrm{ppm}$ of oxidant. Ozone, chlorine, hydrogen peroxide, organic peroxides, and other oxidants provide positive interferences, while reductants including reducing dust cause low results.

Two methods $[33,34]$ have been recommended for determination of total oxidants (ozone), in which the iodine produced is electrochemically reduced. That developed by Mast (and so called) impresses a potential across an electrolytic cell and the amperometric current, proportional to the oxidant-produced iodine, is measured. In the galvanic method of Hersch, the cell is constructed so that spontaneous reduction of the iodine occurs when the cell is shorted, and the current measurement again is related to the oxidant content of the sample. Such methods are essentially continuous, but suffer from the interferences common to all iodimetric procedures. An ozone analyzer based on the chemiluminescent reaction of $\mathrm{O}_{3}$ with an organic dye [1,35] appears to be highly specific. In this technique, a disk coated with Rhodamine $B$ adsorbed on silica gel is exposed to the ozone-containing air and the 
resultant emission is detected by a phototube. The current generated is proportional to the mass of ozone per unit time flowing over the disk. The technique appears to be specific, precise, and has sensitivity comparable with the methods above described but yields significantly higher values. Fundamental studies and better standardization techniques would appear to be needed to clear up the problem.

Several additional chemical methods for the determination of ozone should be mentioned. The Bravo-Lodge technique consists in the ozonolysis of 4-4' dimethylstilbene to produce anisaldehyde which is determined, spectrophotometrically [36]. The chief objection to this method is that it is carried out in a very corrosive solvent. Hauser and Bradley [37] have described a specific ozone technique using 1,2-di-( 4 pyridyl) ethylene as the absorbing medium and colorimetric analysis of the resulting aldehyde. A trans-butene-2 gas phase titration method has been described which involves reaction of the air sample with this hydrocarbon. This reactive olefin selectively removes the ozone which is determined from decrease in the hydrocarbon concentration [38].

One of the earliest techniques used to identify ozone in ambient air is based on the rapid cracking of stressed rubber bands [39]. This technique is useful for survéy work but is hardly quantitative.

e. Volatile Fluorides and Hydrogen Fluoride.

Volatile fluorides in the atmosphere are determined by absorption of the sample in an impinger, distillation to separate the fluoride from interferences, and measurement by a titrimetric or spectrophotometric procedure. The same process may be modified to determine the fluoride content of plant tissue, for example. Direct titration can be used to determine 0.05 to $10 \mathrm{mg}$ of fluoride in a total sample, while the spectrophotometric method has a detection limit of $0.015 \mu \mathrm{g}$ of fluoride. 
Many ions interfere with the method but the most likely ones are aluminum, iron, phosphate, and sulfate. Distillation, diffusion, ion-exchange, or extraction procedures may be required to eliminate such problems [40].

It would appear that ion-selective electrodes would provide improved measurement techniques for fluorides. Such electrodes have been found to be advantageous in measurement of corresponding fluoride levels in other substances and the methods need only be reduced to practice in the pollution field to be useful.

f. Carbon Monoxide. Carbon monoxide may be determined by a method in which it reacts with an alkaline solution of the silver salt of p-sulfaminobenzoic acid to form a colloidal silver suspension which is measured spectrophotometrically [41]. The minimum detectable limit is $5 \mathrm{ppm}$ of $\mathrm{co}$. Acetylene, olefins, $\mathrm{H}_{2} \mathrm{~S}$, and aldehydes in high concentration interfere but may be removed by passage of the air through a silica gel filter containing $\mathrm{HgSO}_{4}$.

Carbon monoxide is widely determined by the non-dispersive infrared method [42]. Measurements can be made with good reliability down to 1 or $2 \mathrm{ppm}$. However, the response is generally non-linear and carbon dioxide, water vapor, and hydrocarbons can provide interferences.

Gas chromatography is emerging as an important tool for co determination and for simultaneous determination of hydrocarbons as well [43]. The method includes a catalytic reduction system that quantitatively converts $\mathrm{CO}$ to $\mathrm{CH}_{4}$ and a flame ionization detector for measurement. Both $\mathrm{CO}$ and $\mathrm{CH}_{4}$ can be determined between concentrations of 0.010 to $200 \mathrm{ppm}$ by suitable sequencing of the sample.

g. Hydrocarbons. Atmospheric hydrocarbons may be determined by various gas chromatographic methods. Total hydrocarbons can be measured with a flame ionization detector and the result reported as a methane-equivalent. Passage of 
the gas through a chromatographic column can be used to separate individual hydrocarbons for their individual determination [44]. Hydrocarbon contents down to 0.01 ppm by volume can be directly measured, while a freeze-trap can be employed to concentrate the sample, thereby extending the detection limit to $0.1 \mathrm{ppb}$.

h. Other Pollutants. Methods have been described for a number of other gaseous pollutants including chlorine, hydrogen chloride, cyanides, and formaldehyde. Discussion of these will be found in references [25-28].

i. Other Techniques. Mass spectrometry has been used with considerable success as a research tool for air pollution studies. Samples may be collected by a cold trap, for example, for laboratory investigation of pollution problems. The analytical mass spectrometer is unique among gas analysis instruments in that it has the potential ability of performing semi-quantitative analyses of the total gaseous pollutant fraction of the atmosphere. It is probably the only analytical instrument which obtains a signal from every gaseous species present in the sample. This fact, however, is also one of the biggest shortcomings of the method because the amount of data received from a single unfractionated sample may be so great as to prevent interpretation. Methods have been described which either allow a fairly accurate qualitative interpretation as to the identification of the individual species or allow determination of the amounts of various classes of substances. For example, it is possible to determine what portion of the atmospheric pollution is due to traffic gases and what portion is due to industrial pollution. Application of special techniques and advanced instrumentation such a fractional distillation, chromatographic separation and high resolution mass spectrometry should allow further refinements of the technique. 
Coulometry is another promising technique which is finding increased use, especially in $\mathrm{SO}_{2}$ determinations. It may well be the most important means for short time-scale calibration of $\mathrm{SO}_{2}$ permeation tubes [45].

Gas chromatography has already been mentioned, and it should be systematically studied for its applicability to pollutant measurements. More selective detectors based on radio-frequency excitation, for example, would appear to be very promising and should be investigated.

The electron-impact spectrometer developed in IBS [46] has very interesting possibilities for adaptation as a gas analytical tool provided its sensitivity can be increased.

2. Particulate Pollutants

Particulates may be discrete and reasonably homogeneous pieces of matter, solid or liquid, crystalline or amorphous, or they may be such materials on which other liquids, solids, or gases are attached or adsorbed. Their analysis is becoming increasingly important. The kinds of analyses required are shown in Table 40 .

a. Dustfall and Sootfall. The amount of dust that settles from the atmosphere is an important index of air pollution. Most of the particles involved will range in size from approximately 1 to $200 \mu \mathrm{m}$ [47] with predominance in the upper range.

Faliout is the most obvious type of pollution, except for odors, lacrymators, and of course, smoke. Dust is collected in buckets over a long period of time. Care is exercised to eliminate obvious foreign matter. For example, a bird ring is installed to provide a preferred spot for their inevitable roosting with minimization of accumulation of droppings in the bucket. Bugs are picked out, as are also leaves. The total collection is weighed and expressed as milligrams $/ \mathrm{cm}^{2} /$ month or tons $/ \mathrm{mile}^{2} /$ month, e.g. It is obvious that such methods must be standardized and the procedures generally 
accepted if the measurements are to achieve any degree of comparability.

It is often desirable to chemically analyze the dust collected as outlined above. A simple separation into water soluble, water insoluble, and benzene soluble fractions, respectively, can give interesting information on the general nature of a collection. More information can be obtained by chemical analysis for metallic elements such as lead, cadmium, iron, calcium, and sodium, for example, and such anions as chloride, fluoride, nitrate, carbonate, and sulfate. The classical techniques of spectrophotometry, polarography, and atomic absorption spectroscopy are generally used for the analyses mentioned above. Spectrochemical analysis is also used extensively. Neutron activation analysis has recently been used for elemental analysis. Free carbon may be determined to indicate soot fall, specific inorganic compounds or materials such as asbestos fibers may be looked for, and specific organic compounds may be isolated. Biological debris such as pollen grains may be sought although such counts are usually made by other procedures [48].

b. Air-borne Particulates. Air-borne particulates are collected by filtration, impaction, impingement, centrifugation, and electrostatic or thermal precipitation [49]. The particle size ranges from $0.01 \mu \mathrm{m}$ to $100 \mu \mathrm{m}$ with predominance in the lower range $[50]$.

Collection by filtering may use a relatively open-pored filter of paper or fiber-glass through which the air is drawn at a rapid rate. Such a "High-Vol" sample may be weighed as a measure of total particulates and/or the deposit may be analyzed as outlined below. The variability of such filters appears to provide problems in the quantitative evaluation of samples collected by this technique so that standard filtering media are urgently needed [51]. Low velocity but highly efficient filters such as Millipore, for example, may 
be used and the collected material analyzed. Such filters show reduced flow rates as the sampling time increases, due to clogging of pores, so that care needs to be exercised to measure the total volume of air flow during a collection period. The use of a limiting orifice helps to provide a reasonable constant flow during the sampling period.

Chemical analysis of filter-collected samples may be carried out as discussed above. Because of uncertainties in the size ranges of particles collected, the exact relation between the analysis of a "High-Vol" sample and that of the particulates in the atmosphere is open to question. It appears that the removal of the sample from the filter media offers some problems and that contamination from the filter media must be considered.

No attempts appear to have been made to obtain complete analyses of material collected by a "High-Vol" sampler. While much analytical data are available, a material accountability of only about 35 percent is ordinarily achieved [52]. Detailed and complete compositional information of samples systematically collected at selected locations and at definite time intervals will become very important as air quality standards for particles become more specific.

Polluted air may contain a large number of organic particulate compounds, arising from incomplete combustion processes. secondary reactions in the air account for still additional compounds. It is important to determine composition as a function of size and to know whether or not specific compounds are adsorbed or attached to other particulates. Particles in the range of 0.2 to $3 \mu \mathrm{m}$ are of special interest since they are said to be most lung damaging [53].

Organic particulates are best collected on filters since electrostatic or thermal precipitation may alter them. Also, impactors may shatter them or otherwise change their physical nature. Since these materials may be bio-active, protection 
from sun and rain during collection and from actinic light during analysis may be required.

Analysis of organic particulate material is almost invariably done by laboratory methods on selected samples [54]. Microscopic or electron-microscopic examinations may be made, followed by separation using the techniques of modern organic analysis, such as extraction, and the chromatographic techniques designated as thin-layer, liquid, and column, respectively. Identification of components and their quantitative determination may be accomplished by use of mass spectrometry, gas chromatography, NMR, and infrared or ultraviolet spectrometry. Such techniques are costly and time-consuming so that analyses must be restricted to special samples.

The complexities of the methodology required for organic particulate analyses are illustrated by consideration of the methods for determination of polynuclear aromatic hydrocarbons assembled by the Intersociety Subcommittee and recently published [55]. In brief, the procedure consists in extraction from the "High-Vol" fiber glass filter with benzene in a Soxhlet extractor. This organic fraction is further separated into 30-40 sub-fractions by means of column chromatography on alumina, using increasing amounts of diethyl ether in pentane as the eluent. Each of these sub-fractions is dried, dissolved in pentane, and spectrophotometrically analyzed. Fluorimetric analysis is used in other cases. Microgram quantities of the following compounds have been determined in this way: anthracene; phenanthrene; fluoranthrene; pyrene; benz [a] anthracene; chrysene; benzo [a] pyrene; benzo [e] pyrene; perylene; benzo [g,h,i] perylene, anthanthrene; and coronene. A complete analysis takes at least two days in the hands of an experienced analyst.

One of the methods described in the series cited above is a microtechnique utilizing alumina thin-layer chromatography. About $3 \mathrm{ng}$ of benzo [a] pyrene can be detected. Because of the 
sensitivity, the laboratory air must be very clean. Cigarette smoking cannot be allowed since it contributes heavily to the background and lessens the sensitivity.

It is clear from the above that detailed information on the composition of organic particulates is an exhausting task. Accordingly, more rapid and/or automated methods are urgently needed. The recently developed technique of liquid chromatography appears to be a promising tool for such determinations and should be investigated.

3. Analysis of Single Particles

The identification of single particles offers valuable information in a number of situations. It provides important clues to the origin of particulate contaminants, perhaps to pinpoint an emission source. Since chemical methods provide information on the total sample, and some size ranges are more important than others, a gross analysis leaves many unanswered questions.

One of the important techniques for particle identification is optical microscopy. Particles with a minimum dimension of $1 \mu \mathrm{m}$ can be observed with optical microscopes. The morphology, color, and general appearance can be very revealing to the trained microscopist. The Particle Atlas [56] contains pictures of literally scores of particles as observed under the microscope and is especially helpful for identification of environmental samples. The optical properties of crystalline material offer means of determination of composition and simple counting of selected species can provide concentration information. Sensitive chemical tests may be made on the microscope stage for qualitative confirmation of composition. Microscopy is valuable for the selective removal of individual particles from a sample for examination by other techniques.

The electron probe microanalyzer is very useful for quantitative analysis of small samples, hence it should find increasing use for particulate analysis. Present models have 
beam diameters of the order of $0.3 \mu \mathrm{m}$ and further reduction of the beam size is to be expected. However, it is possible to obtain useful signals from particles smaller than the beam diameter if they are suitably isolated. The ion-probe microanalyzer has a sensitivity about 100 times greater than the electron probe instrument and hence, would appear to have greatly expanded capabilities for particulate analysis.

$\mathrm{X}$-ray diffraction can be used to identify actual compounds and phases for particulates greater than $2 \mu \mathrm{m}$ in size. Powder patterns of dust samples can be prepared and compared with those of raw materials and with waste from a plant suspected of being an emission source. It should be possible to identify such compounds as solid sulfates and nitrates formed in atmospheric reactions, for example.

Microchemical analysis has made important advances in recent years so that the chemical composition of single particles, isolated by microscopic means, can be determined in some cases. The techniques of spectrophotometry and electrochemistry have special promise in such analyses.

E. Summary

A review such as the present chapter, emphasizes the fact that air pollutant analysis is not a new field and indeed is a well established science. However, the recent high intensity of interest in this subject and the concern for atmospheric improvement is making increasing demands on the analytical chemist. More stringent limits for air quality are coupled with demands for rapid and reliable measurements at low cost. Continuous monitoring methods tied in with data aquisition systems are also needed. These requirements can only be met by a new breed of instrumental techniques. However, many of the classical methods will remain and need to be improved for referee or umpire analyses. 
The fact that new and unevaluated methods are appearing and will be used by technicians rather than trained analysts makes two programs urgent. The first of these concerns method evaluation to identify the superior ones as well as those that are inferior. The National Air Pollution Control Administration has such a program in operation to establish referee methods. The American Society for Testing and Materials is also planning an effort of this kind.

The second program concerns the development of a wide variety of standard reference materials to standardize air pollutant measurements. A modest effort in this direction is already being made at NBS which it is proposed to expand. Because many of the substances measured are reactive or unstable, new concepts of standards, including means to reliably generate such standards as needed, will have to be developed.

In the area of particulates, there is a generally expressed need for a readily available research material that all workers in the field may use for intercomparison of their measurements and techniques. The National Bureau of Standards intends to collect a large sample of particulate matter from the atmosphere, to characterize it as fully as possible, and to make it available to environmental analysts. Such a standard Reference Material should do much to up-grade the science of air pollutant analysis and aid in producing compatible analytical measurements required to insure air quality control.

(J.K. Taylor) 
A. Personnel Listing

John K. Taylor, Section Chief

Carolyn E. Smith, Secretary

Gas Analysis --

Ernest E. Hughes

Julian M. Ives

Gayl Silver (Guest Worker)

Polarography --

E. June Maienthal

Microscopy and Microchemistry --

Rolf A. Paulson

William P. Schmidt

B. Publications

1. J. K. Taylor, Editor, "Microchemical Analysis Section: Summary of Activities July 1968 to June 1969", NBS Technical Note 505, October 1969.

2. J. Knoeck and J. K. Taylor, "Aqueous Boric AcidBorate-Mannitol Equilibria", Anal. Chem., 4l, 1730 (1969).

3. J. Knoeck, "Vibrational Spectrometric and Electrochemical Evidence for Lanthanum (III)-Nitrate Complexes in Aqueous Solution". Anal. Chem., 4l, 2069 (1969).

4. D. H. Freeman, L. A. Currie, E. C. Kuehner, H. D. Dixon, and R. A. Paulson, "Development and Characterization of IonExchange Bead Microstandards", Anal. Chem. 42, 203 (1970).

5. L. Machta and E. E. Hughes, "Atmospheric Oxygen, 1967 to 1970", Science 168, 1582 (1970).

C. Talks

1. J. K. Taylor, "Electrochemical Methods for Trace Analysis", Staten Island Subsection, New York Section, American Chemical Society, Staten Island, New York, October $21,1969$. 
2. J. K. Taylor, "Man and His Environment". Washington Academy of Sciences, Georgetown University, October 26, 1969.

3. J. K. Taylor, "Electrochemical Analysis at the National Bureau of Standards", The Technion University, Haifa, Israel, November 18, 1969.

4. J.K. Taylor, "Electrochemical Analysis at the National Bureau of Standards", University of Geneva, Switzerland, November 21, 1969.

5. J. K. Taylor, "Recent Advances in Electrochemical Analysis", University of Ghent, Ghent, Belgium, November 26, 1969

6. J. K. Taylor, "High Precision Coulometric Analysis", EURATOM Central Bureau for Nuclear Measurements, Geel, Belgium, November 27, 1969.

7. J. K. Taylor, "Electrochemiçal Analysis at the National Bureau of Standards", Imperial College, University of London, London, England, November 28, 1969.

8. J. K. Taylor, "High Precision Coulometric Analysis", Atomic Energy Research Establishment, Harwell, England, December I, 1969.

9. J. K. Taylor, "General Problems of Air Pollutant Analysis", University of Florida, Gainsville, Florida, May 6, 1970.

10. J.K. Taylor, "Relations of Electricity and Chemistry", John F. Kennedy High School, Rockville, Maryland, October 20, 1969.

11. J.K. Taylor, "The Chemical Analysis of Air Pollutants", NBS-NIH Symposium, National Bureau of Standards, Gaithersburg, Maryland, March 10, 1970.

12. J. K. Taylor, "Relations of Electricity and Chemistry", Washington Irving Intermediate School, Springfield, Virginia, March 18, 1970.

13. J.K. Taylor, "Relations of Electricity and Chemistry", High Point High School, Beltsville, Maryland, April 24, 1970.

14. J. K. Taylor, "Careers in Chemistry", Central High School, Capitol Heights, Maryland, May 15, 1970. 
15. J. K. Taylor, "What is Chemistry?", Holy Cross Elementary School, Garrett Park, Maryland, May 22, 1970.

16. J. K. Taylor, "State-of-the-Art of Air Pollutant Analysis", Division 310.00 Scientific Discussion, April 8, 1970.

17. J. K. Taylor, "Standards for Air Pollution Analysis", NAPCA Standards Advisory Committee Meeting, Berkeley, California, April 1, 1970.

D. Committee Activities

J.K. Taylor

Service Analysis Coordinator, Analytical Chemistry Division Member, Review Board, Science Book List, American Association

for the Advancement of Science Elected member, Council of the American Chemical Society (ACS) Secretary, Council Committee on Chemical Education, American

Chemical Society

Member, Panel on Chemistry, Civil Service Board of Examiners Member, Board of Managers, Chemical Society of Washington Member, Advisory Committee, Laboratory Guide, American Chemical

Society

Chairman, Chemical Education Topical Group, Chemical Society of Washington

Chairman, Chemical Education Committee, Chemical Society of Washington

Member, ISO/TC 48 Laboratory Glassware and Related Apparatus Member, Council, Electroanalytical Group, Society for Analytical Chemistry

Vice Chairman, NBS Air Pollution Study Group Member, Advisory Panel, NBS Measurements for Air Quality Program Member, Advisory Panel, Chemical Technology Journal Member, Visiting Committee, GSA National Laboratory

R. A. Paulson

Member, Service Analysis Committee, Analytical Chemistry Division, NBS 
Collaborator, Committee for Study of Microchemical Methods, Association of Official Agricultural Chemists

W. P. Schmidt

Collaborator, Committee for Study of Microchemical Methods, Association of Official Agricultural Chemists

Collaborator, Carbon, Hydrogen and Nitrogen Methods, Commission on Microchemical Techniques, IUPAC

E. June Maienthal

NBS Reporter for Capital Chemist, Chemical Society of

Washington.

Editor, Analytical Chemistry Division Newsletter, NBS.

Chairman, Liaison Committee, Chemical Society of Washington

E. Award

John K. Taylor - Chemical Society of Washington

Service Award for 1969, December 11, 1969

F. Standard Reference Materials Analyses

Material

Microstandard Particles

Bilirubin

Glucose

Special Steels

SRM 1261

SRM 1264

SRM 1265

SRM 361

SRM 365

Lead-Base Bearing

MetaI SRM 1132

Glass

Trace-element Standards Orchard Leaves
Analyst Determination

RAP

Density

Elemental Analysis

WPS

Elemental Analysis Ash

RAP Cl, $\mathrm{SO}_{4}$

M

EJM

$E J M$

EJM

EJM

EJM

$\mathrm{Bi}, \mathrm{Pb}, \mathrm{Te}$

$\mathrm{Bi}, \mathrm{Pb}, \mathrm{Te}$

Ti

$\mathrm{Bi}, \mathrm{Pb}, \mathrm{Te}$

$\mathrm{Ti}$

P

$\mathrm{P}$

P

$\mathrm{P}$

$\mathrm{P}$

EJM $\quad \mathrm{Sb}$

P

EJM Fe, Ti, Ni

P

RAP;

WPS;

EJM

$N, P$

$\mathrm{Al}, \mathrm{Bi}, \mathrm{Fe}, \mathrm{Pb}$

$M, G$

P 
Urea Microchemical Standard SRM 2141

Borax SRM 187b

Disodium Phosphate SRM 186IIC

Hydrocarbon in Air

SRM 1610

SRM 1611

SRM 1612

SRM 1613

Potassium Chloride

SRM

Sodium Chloride
RAP;

WPS N,P

WPS Purity

WPS Purity

JMI; $\mathrm{CH}_{4}$ content

$\mathrm{EEH}$
M , G

Misc.

Misc.

MS, GC 。
Key to Analysts

EJM - E. June Maienthal

RAP - Rolf A. Paulson

WPS - William P. Schmidt
WPS Purity

WPS Purity

Key to Technique

G - Gravimetry

M - Microchemical

P - Polarographic
Misc.

Misc. 


\section{REFERENCES}

[1] R. K. Stevens and A. E. O'Keefe, Anal. Chem. 42, 143A (1970).

[2] A. E. O'Keffe and G. C. Ortmann, Anal. Chem., 38, 760 (1966).

[3] F. P. Scaringelli, S. A. Frey and B. E. Saltzman, AIHA Journal No. 28, 260 (1967).

[4] S. S. Brady and J. E. Chaney, J. Gas Chromatog., 4, 42 (1966).

[5] W. B. Campbell and O. Maass, Can: J. Res., 2, 42 (1930).

[6] C. E. Maass and O. Maass, JACS, 므, 1352 (1928).

[7] Daisaburo Murakami and Niichiro Tokura, Bull. Chem. Soc. Jap., 31, 431 (1958).

[8] F. A. Cotton and G. Wilkinson, "Advanced Inorganic Chemistry", Interscience, (1962) p. 423.

[9] F. A. Quinn, Jr., D. E. Roberts and R. N. Work, J. Appl. Phys., 22, 1085 (1951).

[10] E. E. Hughes, Environ. Sci. Tech., 2, 201 (1968).

[11] M. Shepherd, J.Res. NBS, 26, 351 (1941).

[12] J. K. Taylor, Editor, NBS Technical Note 505, October 1969.

[13] J.K. Taylor, Editor, NBS Technical Note 455, October 1968.

[14] L. Machta and E. E. Hughes, Science, 168, 1582 (1970).

[15] R. Stromberg, et.al., In preparation.

[16] F. A. Pohl, Mikrochim. Ichnoanal. Acta., 1963, 855.

[17] E. J. Maienthal and J. K. Taylor, Anal. Chem., 37, 1516 (1965).

[18] Official Methods of Analysis of the Association of Official Agricultural Chemists, 10th. Edition, 1965, Page 744 .

[19] Ibid, page 747 .

[20] Al Steyermark "Quantitative Organic Microanalysis", 2nd. Edition. page 354 Academic Press 1961. 
[21] J. P. Lodge, Analytical Chemistry, 26, 1831 (1954).

[22] Raleigh Gilchrist, J. Res. NBS, 20, 754 (1938).

[23] J.K. Thorne, Cleveland Refractory Metals, Private Communication.

[24] R. L. Nuttall, A. H. Laufer, M. V. Kilday, Enthalphy of Formation of Ketene. In press.

[25] A. C. Stern, Editor, "Air Pollution", Academic Press, New York (1968).

[26] M. Katz, "Measurement of Air Pollutants", World Health Organization, Geneva, Switzerland (1969).

[27] P. L. Magill, F. R. Holdero and C. Ackley, Editors, "Air Pollution Handbook", McGraw-Hill, New York (1956).

[28] Intersociety Methods, in Health Lab. Science, $\underline{6}$, April (1969), I, January (1970).

[29] Health Lab. Science 7,4 (1970).

[30] M. Katz, in "Air Pollution", A. E. Stern, Editor, Vol. II, p. 53, Academic Press, New York (1968).

[31] M. S. Budd and H. H. Bewick, Anal. Chem. 24 1 , 1536 (1952).

[32] B. E. Saltzman, Anal. Chem., 26, 1949 (1954).

[33] G. M. Mast and H. E. Saunders, ISA Transactions, I, 325 (1962).

[34] P. Hersch and R. Deuringer, Anal. Chem., 35, 897 (1963).

[35] H. V. Renger, J. Geophys. Res., 69, 3795 (1964).

[36] H. A. Bravo and J. P. Lodge, Jr., Anal. Chem., 36, 671 (1964).

[37] T. R. Hauser and D. W. Bradley, Anal. Chem., 39, 1184 (1967).

[38] J. J. Bufalini, Environ. Sci. Technol., 2, 703 (1968).

[39] T. Vega and C. Seymour, J. Air Pollution Control Assoc., II, 28 (1961).

[40] Health Lab. Science 6 , 64 (1969).

[41] Health Lab. Science 7,75 (1970). 
[42] Health Lab. Science I, 81 (1970).

[43] Stevens, R. K. and O'Keefe, A. E., Anal. Chem. 42, Al47 (1970).

[44] Health Lab. Science I, 23 (1970).

[45] F. P. Scaringelli and K. A. Rehme, Anal. Chem. 4l, 707 (1969).

[46] C. E. Kuyatt, Proc. Tenth Symp. of Electronics and Laser Beam Technology, NBS (1969).

[47] P. W. West, in "Air Pollution", A. C. Stern, Editor, Vol. II, p. 148, Academic Press, New York (1968).

[48] J. M. Sheldon, R. E. Lovell and K. P. Matthews, Manual of Clinical Allergy, W. B. Saunders Co., Philadelphia, Pennsylvania (1953).

[49] E. R. Hendrickson, in "Air Pollution", A. E. Stern, Vol. II, pp 3-52, Academic Press, New York (1968).

[50] P. W. West, in "Air Pollution", A. C. Stern, Editor, Vol. II, p. 151, Academic Press, New York (1968).

[51] Private communication

[52] A. P. Altshuller, NAPCA, private communication.

[53] P. Kotin, Cancer Res. 16, 375 (1956).

[54] E. Sawicki, NAPCA, private communication.

[55] Health Lab. Science, I, 31, 45, 56, 60, 68 (1970).

[56] W. C. Mccrone, R. G. Draftz and J. G. Delby, "The Particle Atlas", Ann Arbor Publishers (1967). 
Latest developments in the subject area of this publication, as well as in other areas where the National Bureau of Standards is active, are reported in the NBS Technical News Bulletin. See following page. 


\section{HOW TO KEEP ABREAST OF NBS ACTIVITIES}

Your purchase of this publication indicates an interest in the research, development, technology, or service activities of the National Bureau of Standards.

The best source of current awareness in your specific area, as well as in other NBS programs of possible interest, is the TECHNICAL NEWS BULLETIN, a monthly magazine designed for engineers, chemists, physicists, research and product development managers, librarians, and company executives.

If you do not now receive the TECHNICAL NEWS BULLETIN and would like to subscribe, and/or to review some recent issues, please fill out and return the form below.

Mail to: Office of Technical Information and Publications

National Bureau of Standards

Washington, D. C. 20234

Name

Affiliation

Address

City State Zip

$\square$ Please send complimentary past issues of the Technical News Bulletin.

$\square$ Please enter my 1-yr subscription. Enclosed is my check or money order for $\$ 3.00$ (additional $\$ 1.00$ for foreign mailing). Check is made payable to: SUPERINTENDENT OF DOCUMENTS. TN 545 


\section{PERIODICALS}

JOURNAL OF RESEARCH reports National Bureau of Standards research and development in plysics, mathematics, chemistry, and engineering. Comprehensive scientific papers give complete details of the work, including laboratory data, experimental procedures, and theoretical and mathematical analyses. Illustrated with photographs, drawings, and charts.

Published in three sections, available separately:

\section{Physies and Chemistry}

Papers of interest primarily to scientists working in these fields. This section covers a broad range of physical and chemical research, with major emphasis on standards of physical measurement, fundamental constants, and properties of matter. Issued six times a year. Annual subscription: Domestic, $\$ 9.50$; foreign, $\$ 11.75^{*}$.

\section{Mathematical Sciences}

Studies and compilations designed mainly for the mathematician and theoretical physicist. Topics in mathematical statistics, theory of experiment design, numerical analysis, theoretical physics and chemistry, logical design and programming of computers and computer systems. Short numerical tables. Issued quarterly. Annual subscription: Domestic, $\$ 5.00$; foreign, $\$ 6.25 *$.

\section{Engineering and Instrumentation}

Reporting results of interest chiefly to the engineer and the applied scientist. This section includes many of the new developments in instrumentation resulting from the Bureau's work in physical measurement, data processing, and development of test methods. It will also cover some of the work in acoustics, applied mechanics, building research, and cryogenic engineering. Issued quarterly. Annual subscription: Domestic, $\$ 5.00$; foreign, $\$ 6.25 *$.

\section{TECHNICAL NEWS BULLETIN}

The best single source of information concerning the Bureau's research, developmental, cooperative and publication activities, this monthly publication is designed for the industry-oriented individual whose daily work involves intimate contact with science and technology-for engineers, chemists, physicists, research managers, product-development managers, and company executives. Annual subscription: Domestic, $\$ 3.00$; foreign, $\$ 4.00 *$.

- Difference in price is due to extra cost of foreign mailing.

\section{NONPERIODICALS}

Applied Mathematics Series. Mathematical tables, manuals, and studies.

Building Science Series. Research results, test methods, and performance criteria of building materials, components, systems, and structures.

Handbooks. Recommended codes of engineering and industrial practice (including safety codes) developed in cooperation with interested industries, professional organizations, and regulatory bodies.

Special Publications. Proceedings of NBS conferences, bibliographies, annual reports, wall charts, pamphlets, etc.

Monographs. Major contributions to the technical literature on various subjects related to the Bureau's scientific and technical activities.

National Standard Reference Data Series. NSRDS provides quantitative data on the physical and chemical properties of materials, compiled from the world's literature and critically evaluated.

Product Standards. Provide requirements for sizes, types, quality and methods for testing various industrial products. These standards are developed cooperatively with interested Government and industry groups and provide the basis for common understanding of product characteristics for both buyers and sellers. Their use is voluntary.

Technical Notes. This series consists of communications and reports (covering both other agency and NBS-sponsored work) of limited or transitory interest.

Federal Information Processing Standards Publications. This series is the official publication within the Federal Government for information on standards adopted and promulgated under the Public Law 89-306, and Bureau of the Budget Circular A-86 entitled, Standardization of Data Elements and Codes in Data Systems.
Order NBS publications from:
Superintendent of Documents Government Printing Office Washington, D.C. 20402 
U.S. DEPARTMENT OF COMMERCE

WASHINGTON, D.C. 20230

OFFICIAL BUSINESS 